\title{
Mind the gap
}

Citation for published version (APA):

Woolderink - Kamwendo, M. (2016). Mind the gap: evaluation of an online preventive programme for adolescents with mentally ill or addicted parents. [Doctoral Thesis, Maastricht University]. Datawyse / Universitaire Pers Maastricht. https://doi.org/10.26481/dis.20161216mw

Document status and date:

Published: 01/01/2016

DOI:

10.26481/dis.20161216mw

Document Version:

Publisher's PDF, also known as Version of record

\section{Please check the document version of this publication:}

- A submitted manuscript is the version of the article upon submission and before peer-review. There can be important differences between the submitted version and the official published version of record.

People interested in the research are advised to contact the author for the final version of the publication, or visit the DOI to the publisher's website.

- The final author version and the galley proof are versions of the publication after peer review.

- The final published version features the final layout of the paper including the volume, issue and page numbers.

Link to publication

\footnotetext{
General rights rights.

- You may freely distribute the URL identifying the publication in the public portal. please follow below link for the End User Agreement:

www.umlib.nl/taverne-license

Take down policy

If you believe that this document breaches copyright please contact us at:

repository@maastrichtuniversity.nl

providing details and we will investigate your claim.
}

Copyright and moral rights for the publications made accessible in the public portal are retained by the authors and/or other copyright owners and it is a condition of accessing publications that users recognise and abide by the legal requirements associated with these

- Users may download and print one copy of any publication from the public portal for the purpose of private study or research.

- You may not further distribute the material or use it for any profit-making activity or commercial gain

If the publication is distributed under the terms of Article $25 \mathrm{fa}$ of the Dutch Copyright Act, indicated by the "Taverne" license above, 
MIND THE GAP

Evaluation of an online preventive programme for adolescents with mentally ill or addioted parents

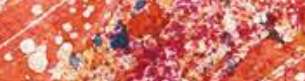
sit.

\section{3its}

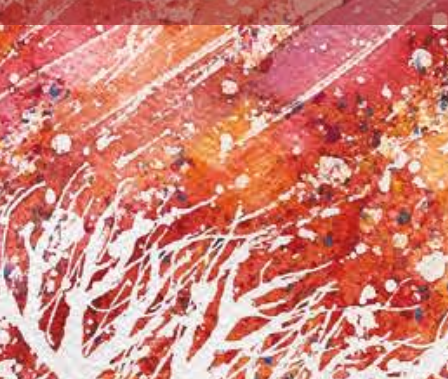

m.

(1)

dist

1.

reton

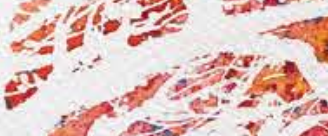

and

- s.

ofing

12.

$\tan 20$

,

1)

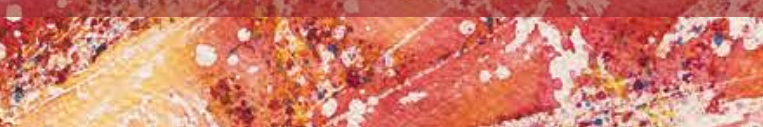
in

IEves

㦴.

रा

32

- 15

$\therefore \nabla$

as

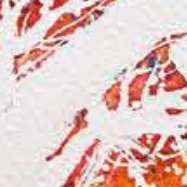

160 in

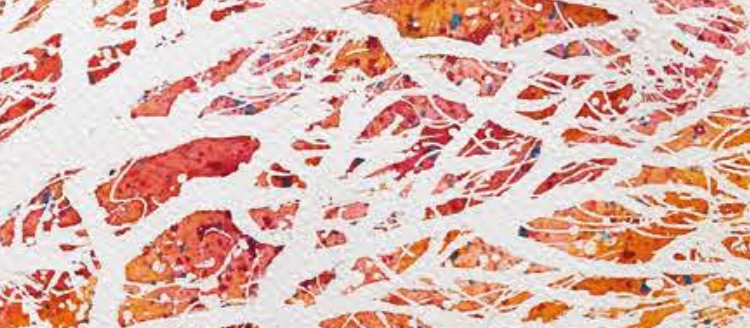

ast.

or 7 s 3

$8050 \%$ :

25.

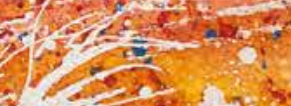

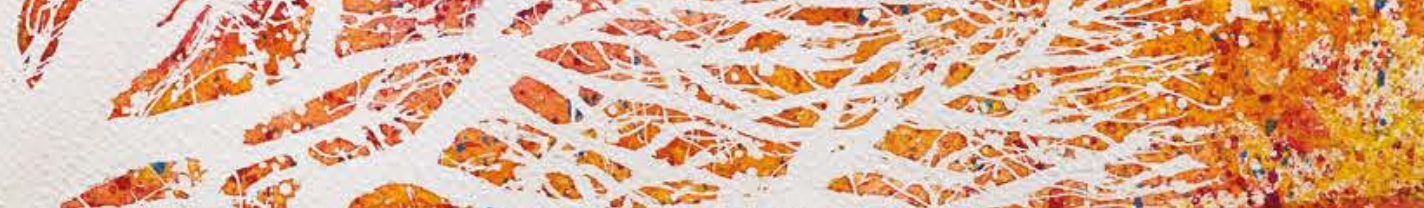

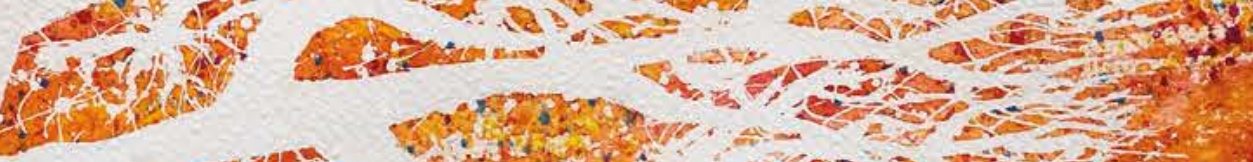

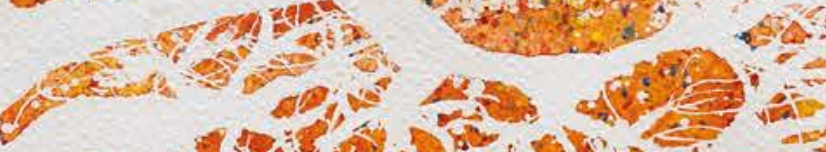

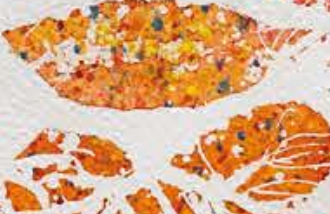

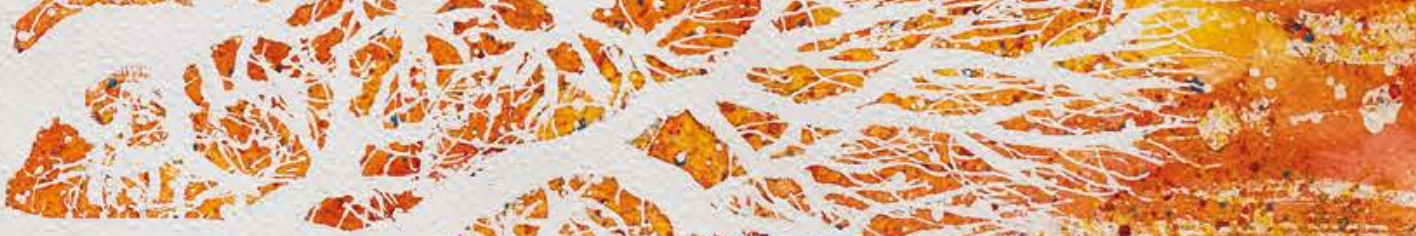

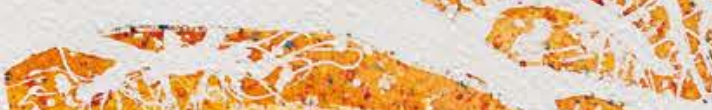


This study was funded by the Netherlands Organisation for Health Research and Development (ZonMw), grant number 200210002. Additional funding to cover the costs for provision of the courses was retrieved by the Innovatiefonds zorgverzekeraars, grant number 2065.

The research presented in this thesis was conducted at the School for Public Health and Primary Care: CAPHRI; at the Department of Clinical Epidemiology and Technology Assessment of the Maastricht Medical Centre and the Department of Health Services Research of Maastricht University. CAPHRI participates in the Netherlands School of Primary Care Research (CaRe).

(C) Marla Woolderink, Maastricht 2016

ISBN: 978-94-6159-624-6

Cover: Amy Wesselink

Layout: Niki Beerens

Printed by: Datawyse, Maastricht

All rights reserved. No part of this dissertation may be reproduced or transmitted in any form or by any means, electronic or mechanical, including photocopying, recording or any information storage or retrieval system, without permission in writing from the author, or, when appropriate, from the publishers of the publications. 


\section{MIND THE GAP}

\section{Evaluation of an online preventive programme for adolescents with mentally ill or addicted parents}

\section{PROEFSCHRIFT}

Ter verkrijging van de graad van doctor aan de Universiteit Maastricht, op gezag van de Rector Magnificus, Prof. dr. Rianne M. Letschert, volgens het besluit van het College van Decanen, in het openbaar te verdedigen op vrijdag 16 december 2016 om 10.00 uur

door

Marla Woolderink 


\section{Promotores}

Prof. dr. C.P. Van Schayck

Prof. dr. F. Smit, VUmc, Amsterdam \& Trimbos Instituut, Utrecht

\section{Copromotor}

Dr. A.D.I. van Asselt, University of Groningen, Groningen

\section{Beoordelingscommissie}

Prof. dr. C.D. Dirksen (voorzitter)

Prof. dr. A.R. Arntz, UvA, Amsterdam

Prof. dr. F.J.M. Feron

Dr. M.H. Nauta, University of Groningen, Groningen

Dr. H.J. Tange 
"Alles wat je aandacht geeft, groeit." Aristoteles 



\section{Table of contents}

$\begin{array}{lll}\text { CHAPTER } 1 \text { General introduction } & 9\end{array}$

CHAPTER 2 Design of an internet-based health economic evaluation $\quad 19$ of a preventive group-intervention for children of partens with mental illness or substance use disorders

CHAPTER 3 An online health prevention intervention for youth with addicted or mentally ill parents:

experiences and perspectives of participants and providers from a randomized controlled trial

CHAPTER 4 Clinical effectiveness of an online preventive programme for adolescents with mentally ill or addicted parents: an online randomized controlled trial

CHAPTER 5 Methodological considerations in service use assessment 83 for children and youth with mental health conditions: issues for economic evaluation

CHAPTER 6 E-research: problems with anonymity and consent

\begin{tabular}{ll} 
CHAPTER 7 & General discussion \\
\hline
\end{tabular}

Summary 123

Samenvatting $\quad 129$

Valorisation $\quad 137$

Appendices 143

Dankwoord $\quad 155$

$\begin{array}{ll}\text { Curriculum Vitae } & 161\end{array}$ 



\section{CHAPTER 1}

General introduction 

The global burden of mental illness is high. Mental illness and substance use disorders are the leading global cause of all non-fatal burden of disease [1, 2]. The World Health Organisation (WHO) reports that the burden of mental disorders continues to grow and it has a significant impact on health, and has economic consequences across the world. Mental disorders place an enormous burden on patients and their social network [3]. This problem becomes even more pressing when the patient suffering from mental illness or addiction is also a parent. Children of parents with mental illness or addiction are at increased genetic, biological and psychosocial risk of developing problems themselves [4-8]. This thesis provides a comprehensive evaluation of an online programme designed to prevent children with mentally ill or addicted parents from developing similar problems.

\section{Prevalence of children who have parents with mental illness and/or addiction}

The lack of international data on the proportion of children who have parents with a mental illness or addiction is a barrier to compare the severity of the problem in different countries; however, there are some national data available. In the Netherlands 400.000 people with children are diagnosed with a mental illness or addiction every year [9]. These parents have a total of 577.000 children younger than 18 years. The Netherlands is a small country and these children represent $17 \%$ of the total child population $[10,11]$, or one in every six children. This percentage does not include children over 18 years old who have to deal with a parent with mental illness or addiction. The estimate is also a conservative one, because the national survey on which it is based only included population data for a selection of mental health disorders (mood disorders, anxiety disorders, ADHD and alcohol and/or drug dependency) and so it does not include the children of parents with other mental health problems (e.g. eating disorder or personality disorders and some other mental illnesses). Other northern European countries such as Sweden and Norway have also published estimates of the proportion of children who have parents with mental illness or addiction. The Norwegian Institute of Public Health estimated in 2010, that $37.3 \%$ of children in Norway had at least one parent who had suffered from mental illness in the past year [12]. In Sweden a national report estimated that $7.8 \%$ of children born in 1987-1989 had a biological parent who had received inpatient treatment for a mental health disorder or addiction. In addition it was also estimated that, for this cohort of children, 26,000 children under the age of 18 year had at least one parent who was hospitalised because of mental illness or addiction [13]. These numbers do not include parents with less severe mental health or addiction problems who did not receive hospital care. In the United Kingdom a large national survey found that around $9-10 \%$ of women and $5-6 \%$ of men are parents currently experiencing mental illness. The same national survey found that as many as one in four children aged 5-15 years had mothers who were at increased risk of developing a mental illness [14]. Comparable numbers of prevalence have also been published elsewhere. In Canada a national survey found that one in six children 
under the age of 12 years, $(570,000$ children) were living in households where one member (most likely a parent) met the criteria for one or more mood, anxiety or substance use disorders. This corresponds to $21.1 \%$ of children in this age group [15]. In Canada the proportion of older children with a parent suffering from mental health or addiction problems has not been calculated. In Australia population estimates indicate that $23.3 \%(1,082,403$ children) of all children have a parent with mental illness, excluding substance use disorders [16]. In most countries the surveys show how pressing the problem of parental mental illness is, given that the prevalence is very likely to be underestimated due to restrictive criteria for the type and severity (whether or not inpatient treatment is required) of parental illness and age of children included in the statistics. Even these conservative estimates show that a large number of children have a parent with a mental illness or an addiction.

\section{Risk, exposure and impact}

There is a vast body of evidence showing that a child who is exposed to parental psychiatric disorders or addiction is at increased risk (genetic, biological and psychological) of developing the same condition as the parent, or another mental illness or addiction problems [4, 5, 17-19]. It is reported that children of parents with mental illness or addiction are not only at higher risk of developing psychiatric disorders, but also at higher risk of developing behavioural problems or other related problems [4-6, 17, 2027]. At all ages these youngsters are at risk; in younger years they are at risk of neglect or abuse, which may lead to emotional or behavioural problems. At later ages they are at risk of developing serious mental health problems (e.g. depression or eating disorders). The children of mentally ill parents are more likely to use professional mental healthcare services and suffer more physical illness than their peers [28]. Monitoring and providing help for these children during adolescence, may be particularly important as this is a transitional period during which many physical, mental and socioaffective changes take place and many disorders first manifest at this time [29].

\section{Interventions for children of mentally ill and/ or addicted parents}

Evidence of the increased genetic, biological and psychological risks faced by children of parents with mental health or addiction problems has resulted in the development of several intervention programmes in the Netherlands and other countries. Preventive interventions are intended to prevent the onset of a mental illness, whereas curative interventions are intended to treat problems once they have manifested or been diagnosed [30, 31]. There are several types of preventive interventions; universal interventions targeting the whole population, selective interventions targeting at risk groups, populations whose risks of becoming ill is above average and indicated interventions which target individuals who a had manifestation of specific risk factors [32].This thesis focuses on an intervention classified as a selective preventive interven- 
tion, targeted at children at risk of developing mental illness or addiction because of parental mental illness or addiction.

Several educational websites have been developed to enable the children of mentally ill or addicted parents to find information, seek help or find peers in a similar situation. In addition, there are courses and programmes (interventions) targeting this population up to the age of 25 years old. For the youngest group there are family interventions and for adolescents there are face-to-face support groups. A complete list of Dutch interventions for children with mentally ill or addicted parents is provided in Appendix 1. This thesis focuses on the population of adolescents (defined as those aged 16-25 years old) with parents with mental illness and/ or addiction and on the online Kopstoring course. Kopstoring has recently attracted the attention of experts in other countries such as Sweden and United Stated of America.

\section{Kopstoring: an online preventive course}

In 2006 the Kopstoring course was launched for adolescents from 16 to 25 years old with a parent with a mental illness or addiction. Kopstoring was developed at the Trimbos Institute and is embedded in an informative website, www.Kopstoring.nl, which provides several other services as well as the Kopstoring programme. Kopstoring consists of eight consecutive weekly online meetings using a 'chatbox' and a ninth meeting intended for evaluation. The aim of the intervention is to strengthen protective factors, such as self-management skills and psychological wellbeing, and thus prevent the development of psychological disorders. The experts (prevention workers and psychologists from mental health care institutions) involved in the delivery of the intervention are specialists in problems related to having a mentally ill or addicted parent and in prevention of mental health problems in young people. The intervention is protocol-driven and highly structured. The themes of the eight meetings are as follows: getting acquainted with the home situation, roles in families, thoughts and feelings, questions about addiction and mental health problems, different styles of behaviour, social networks and leading your own life: social networks and the future. Participants are required to carry out homework assignments every week [33]. 


\section{Data used in this thesis}

Data was collected from an online multi-centre, randomized controlled trial, performed in the Netherlands. The first steps taken, were recruitment and enrolment of offspring (in the 16-25 years age group) of parents with psychological or addiction problems. Recruitment has been carried out by the Trimbos Institute (Netherlands Institute of Mental Health and Addiction) and Maastricht University in close collaboration with and nine mental health services providers ${ }^{1}$. Several online and offline recruitment strategies have been used to reach this vulnerable population. After recruitment, enrolment has taken place and screening was carried out by professionals of the nine mental health services providers. The second step was random allocation of the participants to either the Kopstoring course or a six-month waiting list control group. After these steps, baseline and follow-up measurements were conducted to collect data for the evaluation in this thesis. Additional qualitative data about service utilisation on youngsters in need of mental health care was collected through in-depth interviews with experts from the Netherlands, the United Kingdom and the United States of America.

\section{Research context}

The context in which the studies included in this thesis were performed, was very complex. Data was collected in a period of financial pressure on the youth mental health care system and on the mental health services providers in the Netherlands. In addition, the online evaluation study and the online context of Kopstoring interfered with the requirement of obtaining written consent of participants, a statutory provision of the Dutch Medical Research Involving Human Subjects Act (WMO). Third, obtaining parental (in this case from the patient) written consent to approve their child's participation in the evaluation study influenced the recruitment and enrolment of participants in the evaluation study.

\section{Financial pressure}

In 2010, the year before the start of the randomized controlled trial, two Kopstoring courses were provided (with 7 participants in total). Provision of the courses was funded by the mental health services providers. At the start of the research project, there was no funding available to reimburse provision of the Kopstoring course. Mental health service provides were under so much pressure that two mental institutions ${ }^{2}$ were forced to quit providing Kopstoring courses. At the same time (2011) the number of participants for the Kopstoring courses ascended to 31. Therefore, the need to attract new mental health service providers and funding for provision of the course was deemed necessary. During the course of the randomized controlled trial courses were

\footnotetext{
${ }^{1}$ GGNet, Dimence (recently mindfit), GGZ Oost Brabant, Reinier van Arkel (recently Ypse), GGZ NML (recently VVGI) \& Riagg Zuid, Context, Brijder, Optiment (recently Eleos)

${ }^{2}$ Gelderse Roos (recently Indigo) \& De Grote Rivieren
} 
funded by the Innovatiefonds zorgverzekeraars [34], which allowed the research team and the providers to search and recruit additional mental health services providers to provide the Kopstoring course. Nevertheless, the financial pressure on the professionals and managers was enormous and there was a continuous risk of losing professionals due to this financial pressure. The financial burden caused a lot of turmoil for the professional providers and complicated the recruitment process of the evaluation study.

\section{Online intervention and evaluation}

The evaluation study was designed in such a way that interference with the provision of the Kopstoring course was avoided. Data for the evaluation study was, in accordance with the intervention, collected entirely online. Registration, screening, enrolment, randomisation and measurements were all performed online. In the case of the Kopstoring evaluation, the study was subject to the Dutch Medical Research Involving Human Subjects Act (WMO), which required a written signed informed consent from participants [18-25] and in addition for minors (16 and 17 year old) a written informed consent from the parents or legal guardians. Obtaining written informed consent instead of online informed consent for the evaluation study, and not for the Kopstoring course itself, caused a situation in which participating in the Kopstoring course (not the evaluation study) was preferable over participating in both the course and evaluation. Participating in both would force them to send written forms for the evaluation study while for the Kopstoring course online consent was sufficient for participation. In addition, participants aged 16 and 17 year old, able to give legally binding (online) consent for participation in the Kopstoring course, needed to send back their written informed consent forms and written informed consent forms of their parents or legal guardian. The requirement of obtaining written informed consent in an entire online health setting, created a situation in which participating in the evaluation study of Kopstoring would actually yield many disadvantages. This complicated the enrolment of participants in the randomized trial.

These contextual problems led the research team to make modifications to the trial design during the trial period. 


\section{Objectives of this thesis}

The overall objective of this thesis is to evaluate the clinical, methodological and judicial perspectives and participants and providers perspectives of the online Kopstoring course for adolescent children of parents with mental illness and/ or addiction.

\section{Outline of this thesis}

The first chapter of this thesis (Chapter 2) describes the design of this online, multicentre trial. Chapter 3 reports a process evaluation of participants' and providers' experience and perspectives. Chapter 4 describes the short term clinical effectiveness of the Kopstoring course, comparing the course with a waiting list control condition. Chapter 5 and 6 elaborate on problems encountered with the context of online interventions and evaluation. Chapter 5 assesses problems with identifying and measuring service use of children and youngsters and other factors relevant to economic evaluations for young people with mental health problems. Chapter 6 describes important legal and ethical problems which make it nearly impossible to carry out online evaluation research with vulnerable young people or on sensitive health problems. Chapter 7 provides a discussion of the main findings and elaborates on the most important strengths and limitations of the research followed by recommendations for policy- and decision makers and future research. 


\section{REFERENCES}

1. Whiteford HA, Degenhardt L, Rehm J, Baxter AJ, Ferrari AJ, Erskine HE, et al. Global burden of disease attributable to mental and substance use disorders: findings from the Global Burden of Disease Study 2010. Lancet (London, England). 2013;382(9904):1575-86.

2. Degenhardt L, Whiteford HA, Ferrari AJ, Baxter AJ, Charlson FJ, Hall WD, et al. Global burden of disease attributable to illicit drug use and dependence: findings from the Global Burden of Disease Study 2010. Lancet (London, England). 2013;382(9904):1564-74.

3. Maurin JT, Boyd CB. Burden of mental illness on the family: a critical review. Archives of psychiatric nursing. 1990;4(2):99-107.

4. Weissman MM, Fendrich M, Warner V, Wickramaratne P. Incidence of psychiatric disorder in offspring at high and low risk for depression. Journal of the American Academy of Child and Adolescent Psychiatry. 1992;31(4):640-8.

5. Weissman MM, Wickramaratne P, Nomura Y, Warner V, Pilowsky D, Verdeli H. Offspring of depressed parents: 20 years later. The American journal of psychiatry. 2006;163(6):1001-8.

6. Downey G, Coyne JC. Children of depressed parents: an integrative review. Psychological bulletin. 1990;108(1):50-76.

7. van Loon L, van de Ven MOM, van Doesum KTM, Hosman CMH, Witteman CLM. Negative thoughts and feelings about parental mental illness and the relation with problem behavior in adolesence: an exploratory study. Journal of Child and Family Studies; Epub 2013.

8. Rasic D, Hajek T, Alda M, Uher R. Risk of mental illness in offspring of parents with schizophrenia, bipolar disorder, and major depressive disorder: a meta-analysis of family high-risk studies. Schizophrenia Bulletin. 2014;40(1):28-38.

9. van der Zanden R, Meeuwissen J, Havinga P, Meije D, Konijn C, Beentjes M, et al. Kinderen van ouders met psychische problemen (KOPP) in de jeugdhulp. Utrecht: Trimbos Insituut, 2014.

10. de Graaf R, ten Have M, van Dorsselaer S. De psychische gezondheid van de Nederlandse bevolking. Nemesis-2: Opzet en eerste resultaten. Utrecht: Trimbos-Instituut, 2010.

11. Goossens F, van der Zanden R. Factsheet KOPP/KVO (Prevention factsheet: Children of mentally ill or addicted parents). In: Trimbos-Instituut, editor. Utrecht2012.

12. Rognmo K, Torvik FA, Ask H, Roysamb E, Tambs K. Paternal and maternal alcohol abuse and offspring mental distress in the general population: the Nord-Trondelag health study. BMC Public Health. 2012;12:448.

13. Hjern A, Machica H. Barn som anhöriga till patienter i vården - hur många är de? - Rapport 1 från projektet "Barn som anhöriga" - en kartläggning. 2014.

14. Parker G, Beresford B, Clarke S, Pitman R, Gridley K, Pitman, et al. Research Reviews on prevalence, detection and interventions in parental mental health and child welfare: Summary report. York: Social Policy Research Unit, University of York, 2008.

15. Bassani DG, Padoin CV, Philipp D, Veldhuizen S. Estimating the number of children exposed to parental psychiatric disorders through a national health survey. Child and adolescent psychiatry and mental health. 2009;3(1):6.

16. Maybery D, Reupert A, Patrick K, Goodyear M, Crase L. Prevalence of parental mental illness in Australian families. Psyciatric bulletin. 2009;33:4.

17. Barnow S, Spitzer C, Grabe HJ, Kessler C, Freyberger HJ. Individual characteristics, familial experience, and psychopathology in children of mothers with borderline personality disorder. Journal of the American Academy of Child and Adolescent Psychiatry. 2006;45(8):965-72.

18. Hosman $\mathrm{CMH}$, van Doesum KTM, van Santvoort F. Prevention of emotional problems and psychiatric risks in children of parents with a mental illness in the Netherlands: I. The scientific basis to a comprehensive approach. Australian e-Journal for the Advancement of Mental Health. 2009;8. 
19. Maybery D, Ling L, Szakacs E, Reupert A. Children of a parent with a mental illness: perspectives on need. Australian e-Journal for the Advancement of Mental Health. 2005;4:11.

20. Beardslee WR, Gladstone TR, O'Connor EE. Transmission and prevention of mood disorders among children of affectively ill parents: a review. Journal of the American Academy of Child and Adolescent Psychiatry. 2011;50(11):1098-109.

21. Chassin L, Curran PJ, Hussong AM, Colder CR. The relation of parent alcoholism to adolescent substance use: a longitudinal follow-up study. Journal of abnormal psychology. 1996;105(1):70-80.

22. Dean K, Stevens H, Mortensen PB, Murray RM, Walsh E, Pedersen CB. Full spectrum of psychiatric outcomes among offspring with parental history of mental disorder. Archives of general psychiatry. 2010;67(8):822-9.

23. Hans SL, Auerbach JG, Styr B, Marcus J. Offspring of parents with schizophrenia: mental disorders during childhood and adolescence. Schizophrenia Bulletin. 2004;30(2):303-15.

24. Leverton TJ. Parental psychiatric illness: The implications for children. Current Opinion in Psychiatry. 2003;16:7.

25. Schreier A, Wittchen HU, Hofler M, Lieb R. Anxiety disorders in mothers and their children: prospective longitudinal community study. British Journal of Psychiatry. 2008;192(4):308-9.

26. Singh MK, DelBello MP, Stanford KE, Soutullo C, McDonough-Ryan P, McElroy SL, et al. Psychopathology in children of bipolar parents. Journal of Affective Disorders. 2007;102(1-3):131-6.

27. Stein A, Woolley H, Cooper S, Winterbottom J, Fairburn CG, Cortina-Borja M. Eating habits and attitudes among 10-year-old children of mothers with eating disorders: longitudinal study. British Journal of Psychiatry. 2006;189:324-9.

28. Olfson M, Marcus SC, Druss B, Alan Pincus H, Weissman MM. Parental depression, child mental health problems, and health care utilization. Medical Care. 2003;41(6):716-21.

29. Costello EJ, Mustillo S, Erkanli A, Keeler G, Angold A. Prevalence and development of psychiatric disorders in childhood and adolescence. Archives of general psychiatry. 2003;60(8):837-44.

30. van den Berg M, Schoemaker CG. Effecten van Preventie- Deelrapport vn de VolksgezondheisToekomst Verkenning 2010; van gezond naar beter. Houten: 2010.

31. Ministerie van Volksgezondheid. Nationaal Kompas [cited 2015 07-09-2015]. Available from: www.nationaalkompas.nl.

32. Mrazek PJ, Haggerty RJ. New directions in definitions. 1994.

33. van de Verdoold S, van Dijke, van der Zanden, R. Opgroeien bij ouders met psychische of verslavingsproblemen. Kopp-cursus 16 t/m 25 jaar. Utrecht: Trimbos Instituut, 2007.

34. Innovatiefonds Zorgverzekeraars. http://www.innovatiefondszorgverzekeraars.nl/ Zeist2016 [cited 2016 12-02-2016]. 


\section{CHAPTER 2}

Design of an internet-based health economic evaluation of a preventive group-intervention for children of parents with mental illness or substance use disorders

Woolderink M, Smit F, van der Zanden R, Beecham J, Knapp M, Paulus A, Evers S (2010) Design of an internet-based health economic evaluation of a preventive groupintervention for children of parents with mental illness or substance use disorders. BMC Public Health. 2010 Aug 10;10:470. doi: 10.1186/1471-2458-10-470. 


\section{ABSTRACT}

Background: Preventive interventions are developed for children of parents with mental and substance use disorders (COPMI), because these children have a higher risk of developing a psychological or behavioural disorder in the future. Mental health and substance use disorders contribute significantly to the global burden of disease. Although the exact number of parents with a mental illness is unclear, the subject of mentally ill parents is gaining attention. Moreover, there is a lack of interventions for COPMI-children, as well of (cost-) effectiveness studies evaluating COPMI interventions. Innovative interventions such as e-health provide a new field for exploration. There is no knowledge about the opportunities for using the internet to prevent problems in children at risk. In the current study we will focus on the (cost-) effectiveness of an online health prevention program for COPMI-children.

Methods/Design: We designed a randomized controlled trial to examine the (cost-) effectiveness of the Kopstoring intervention. Kopstoring is an online intervention for COPMI-children to strengthen their coping skills and prevent behavioural and psychological problems. We will compare the Kopstoring intervention with (waiting list) care as usual. This trial will be conducted entirely over the internet. An economic evaluation, from a societal perspective will be conducted, to examine the trial's costeffectiveness. Power calculations show that 214 participants are needed, aged 16-25. Possible participants will be recruited via media announcements and banners on the internet. After screening and completing informed consent procedures, participants will be randomized. The main outcome is internalizing and externalizing symptoms as measured by the Youth Self Report. For the economic evaluation, healthcare costs and costs outside the healthcare sector will be measured at the same time as the clinical measures, at baseline, 3, 6 and 9 months. An extended measure for the intervention group will be provided at 12 months, to examine the long-term effects. In addition, a process evaluation will be conducted.

Discussion: Recent developments, such as international conferences and policy discussions, show the pressing need to study the (cost-) effectiveness of interventions for vulnerable groups of children. This study will shed light on the (cost-) effectiveness of an online preventive intervention. 


\section{BACKGROUND}

This article describes the design and methods of a randomized controlled trial (RCT) evaluating the (cost-) effectiveness of an online digital preventive intervention for children of parents with a mental disorder or a substance use disorder. In Dutch this group is known as KOPP-children (Kinderen van Ouders met Psychiatrische Problemen). In English these children are often referred to as COPMI (Children of Parents with Mental Illness). Even in their adulthood, COPMI-children are regarded as vulnerable.

The prevalence of mental illness is increasing, worldwide [1]. Accordingly, some of the newly diagnosed patients are parents. Although international statistics about global prevalence are not yet available, the subject of mentally ill parents is gaining attention [1]. In the Netherlands every year about 864,000 parents meet the criteria for a DSM III (axis one) mental illness diagnosis [2]. In 2006 about 1.6 million COPMI-children, younger than 22 years, were living in the Netherlands [3], among a total Dutch population of 16 million. More than 700,000 of these children are adolescents between 16 and 25 years old, living with at least one parent who suffers from a mental health or substance use disorder. Internationally, the prevalence and incidence of COPMI is still unknown. The problems encountered by COPMI-children have a large impact on their lives, their social environment and on society as a whole. COPMI-children have, overall, a lower quality of life, and are likely to use health care and social care facilities frequently. In addition participation in school and work can be a problem [4]. Previous studies in this field showed that COPMI-children have $50 \%$ likelihood of developing mental health problems themselves throughout life [5]. If a child has two (biological) parents with mental health or substance use disorders, the likelihood rises to $66 \%$ [3]. During their adolescence, two out of three COPMI-children experience serious problems in coping with a parental manifestation of mental or substance use disorder [4]. There is a growing interest in COPMI children, both nationally, as well as internationally. Recently, an international conference about the forgotten child, stressed the importance of children of parents with mental illness [1]. COPMI have also come to the attention of politicians. In May 2009, for example, members of the Dutch parliament asked formal questions in the House of Representatives about the increasing prevalence of COPMI-children, the consequences of their situation and what solutions were needed. Thus, societal impact and political interest is growing nationally and internationally.

To date, only two studies have examined the effectiveness of COPMI interventions. As a consequence most of the interventions developed for this group have not yet been evaluated and there is no evidence relating their cost-effectiveness [6]. 
Two COPMI interventions used on a large scale in the Netherlands and elsewhere are based on face-to face psycho-educative family intervention. Literature shows that these psycho-educative interventions are effective in terms of protective factors $[7,8]$.

In spite the elevated risks, only a few prevention programs are offered for COPMIchildren and these reach only part of the target population. One of the options for improving accessibility is providing internet-based interventions (E-programs). In the Netherlands an E-program called Kopstoring has recently been developed.

In brief the Kopstoring intervention is an online 8-week educative course which strengthens the coping skills of COPMI-children, and provides knowledge about their parents' mental illness. In a secure chat box COPMI-children work through the themes of the course. Kopstoring is an online intervention for COPMI children aged 16 to 25 years.

In this study we will examine the (cost-) effectiveness of the Kopstoring E-program for COPMI-children. To our knowledge this is the first study to assess the (cost-) effectiveness of E-programs for COPMI-children. Besides the information regarding costeffectiveness, this study will also yield information about COPMI-children's use of healthcare resources.

This study builds on an existing COPMI pilot study which assesses the effects of the online intervention [9]. E-mental health is a relatively new method for delivering interventions in the health care sector at affordable costs. A series of recent studies [10] have demonstrated the positive effects of E-mental health interventions and highlighted features such as therapists being able to delegate routine aspects of their work to computers, thus freeing scarce resources for those patients most in need of it, and delivering interventions in a well-structured way based on the best available evidence [11]. Several systematic reviews $[12,13]$ have identified the effectiveness of E-mental health interventions. Despite a promising trend several authors stresses that both the effectiveness as well as the costs-effectiveness of E-mental health interventions need to be assessed $[12,14]$

In brief, the current study aims to examine the beneficial effects of online intervention for COPMI-children, weighing these against health and societal costs. Potentially, a preventive Kopstoring program will reduce symptoms, behavioural and serious psychological problems, strengthen the COPMI-children's emotional and social functioning and coping skills, and improve their relationship with the ill parent. A decrease in the consumption of health care may follow. Production losses, leaving school prematurely, vandalism and costs related to service use outside the health care sector (social care) are also expected to be lower. This study will lead to more solid evidence-based information about the Kopstoring program. This study will be the first to provide insight into the cost-effectiveness of a preventive E-program for COPMI-children. 


\section{METHODS/DESIGN}

The design will be a multi-centre RCT. The project will consist of an effectiveness study, an economic evaluation, and a process evaluation.

\section{Objective and research questions}

The main study objective is to evaluate the effects, cost-effectiveness and the process of providing an internet-based preventive intervention for COPMI-children from 16-25 years old.

The main research question is as follows: From a societal perspective, is the online psycho-educative prevention program Kopstoring a cost-effective program for reducing the symptoms of children of parents with mental or substance use disorders? In addition, is the intervention delivered in time and according to prevailing standards, according to the protocol and does it meet the expectations of caregivers and participants involved?

The following sub-questions can be identified for the effectiveness study, the economic evaluation study and process evaluation:

\section{Effectiveness study}

1. Does the preventive online intervention reduce the child's internalizing and externalizing symptoms and prevent behavioural and coping problems which are due to the parent's mental illness?

2. Does the preventive online intervention strengthen the child's emotional and social functioning and coping skills?

3. Does the preventive online intervention establish a higher quality of life for COPMI-children?

\section{Economic evaluation study}

1. From a societal perspective, is the delivery of the Kopstoring intervention, in comparison with waiting-list care as usual, preferable in terms of costs, effects and utilities? 


\section{Process evaluation}

1. Is the intervention delivered in time and does it meet accepted standards, according protocol?

2. Is the protocol being followed?

3. What do participants expect from the intervention and what do they believe about the effect of the intervention (credibility and expectancy)?

\section{Design}

This study will consist of an effectiveness study, an economic evaluation study and a process evaluation.

The basis of the effectiveness study will be a pragmatic randomized controlled trial (RCT), in which the Kopstoring program will be compared with a waiting list control condition which reflects care as usual (CAU). The interventions last 8 consecutive weeks with a ninth meeting after a month. Figure 1 presents the flow chart for the study. Cost and outcome measures will be taken at baseline and at 3 months and 6 months after baseline in both conditions. An extended follow-up at 12 months will be conducted in the intervention arm of the trial, to monitor the long-term costs and effects of the intervention.

In the economic evaluation we will compare incremental costs and incremental outcomes of the Kopstoring intervention in relation to care as usual. This economic evaluation will involve both a cost-effectiveness analysis (CEA) and a cost-utility analysis (CUA). The primary outcome measure for the economic evaluation will be the prevention of the development of symptoms over the 6 months of follow-up. Within the costutility analysis, outcomes will be measured by means of the standard Dutch version of the EuroQol. In the CEA, the incremental cost-effectiveness ratio (ICER) will be expressed as the incremental costs per point improvement on the Youth Self Report Questionnaire. The primary outcomes measured for the cost-utility analysis will be QALYs, based on the EuroQol utility scores. $[15,16]$. In the CUA, the ICER will be expressed as the incremental cost per QALY. This economic evaluation will take a societal perspective, including all relevant costs and outcomes.

\section{Process evaluation}

During the study we will include a process evaluation in which we assess several aspects concerning the process of the execution of the intervention and how it is perceived by caregivers and the recipients. We want to identify whether the execution of the RCT is going according to protocol and if so, whether the intervention is meeting accepted standards [17]. We will use qualitative methods to derive this information. We will interview the experts (caregivers) who are involved in the delivery of the Kop- 
storing program to gain knowledge about the factors which harm or stimulate working according to protocol. The recipients will receive a short questionnaire with items about aspects of the intervention.

Credibility and expectations about internet interventions and specifically the Kopstoring intervention will be measured at baseline and after receiving the internet intervention. These results will be used for the process evaluation to gain knowledge about what respondents expect from this internet therapy and how they rate it in terms of credits. Caregivers will also be asked to fill in the Credibility and Expectancy Questionnaire. In addition, the statistics linked to the database system can be useful in determining whether the protocol was followed.

\section{Recruitment population}

Inclusion: Young people 16-25 years old of parents with mental disorder or substance use disorder and no diagnosed disease according DSM IV, able to use a computer and fluent in Dutch

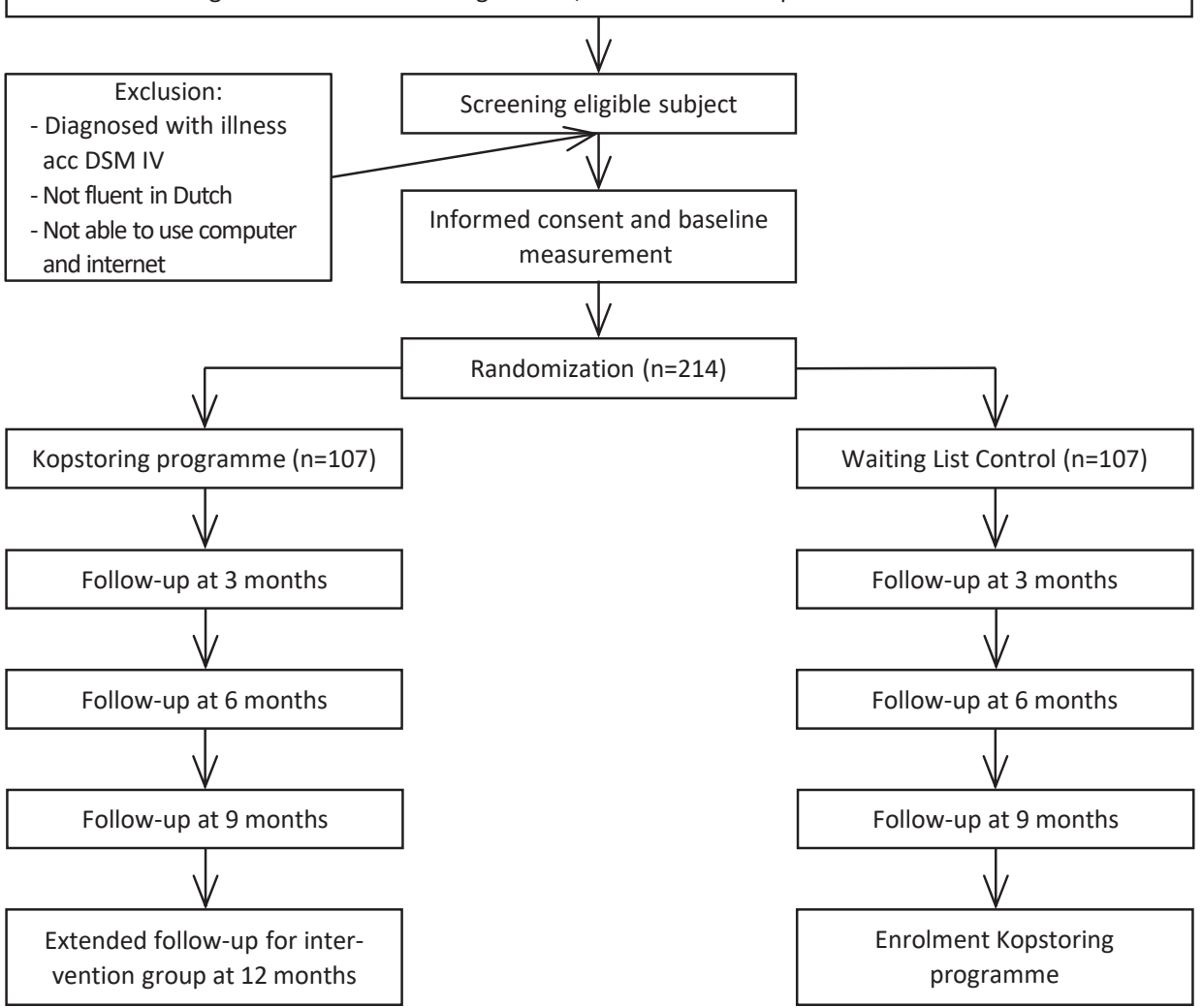

Figure 1: Flow chart study design 


\section{Target population}

The target group is defined as young people aged between 16 and 25 years who have at least one parent suffering from a psychiatric disorder, multiple psychological disorders or an addiction problem. For inclusion in our study, young people have no internalizing and externalising problems. This will be defined using the Youth Self Report List (YSR); the cutoff scores for girls will be a score of 21 and for boys a score of 16 . If the young person has a score above the cutoff score, they will be referred for treatment or other services. In addition, the young person has to be sufficiently fluent in the Dutch language, have access to the internet and be able to use a chat box, to participate actively and be able to listen to other participants. For young people aged 1617 informed consent from the parent is also required. For those aged 18 and over, informed consent of the adolescent is sufficient. Those who are younger than 16 years or older than 25 years are excluded from our study.

\section{Recruitment}

COPMI-children will be recruited in several ways. First, they can be recruited through the online Kopstoring programme (http://www.Kopstoring.nl). This open website has information about the study and its objectives. Second, the mental health institutions who are cooperating in this project and provide the online programme give information about the Kopstoring programme to potential respondents and can recruit young people through their websites, databases, networks and contacts. General practitioners (GPs) will be informed about the study so they can also preselect eligible respondents. There will also be banners and links created for the internet. Public Relations (PR) will be led by the Trimbos Institute which has experts and experience in the field of recruitment and PR.

\section{Intervention}

The Kopstoring programme consists of 8 consecutive online weekly chat group meetings and a ninth evaluation meeting in a chat box at the website http://www. Kopstoring.nl. The aim of the intervention is to strengthen protective factors, such as selfmanagement skills and psychological well-being, and prevent the development of psychological disorders. The experts (prevention workers and psychologists from mental health care institutions) involved in the delivery of the intervention are specialized in the field of COPMI problems and in the prevention of mental health problems in young people. The intervention is protocoldriven and well-structured. There are 8 themes, so each week there is a new theme. The themes are; getting acquainted with the home situation, roles in families, thoughts and feelings, questions about addiction and mental problems, different styles of behaviour, social networks, leading your own life in relation to social networks and what is coming up in the future. The participants are required to carry out homework assignments before entering the chat box (chat 
room) meeting [18]. The Kopstoring programme is part of an informative website which also provides an e-mail service and chat options, alongside the Kopstoring programme.

\section{Care as usual in the Netherlands}

The control condition in this trial is a waiting list with unrestricted access to care as usual. Usual care in the Netherlands is a mix of several services. In this study, care as usual is not standardized and there are no protocols. Young people can request a lot of services: for example, a general practitioner, a psychologist, or mental health or institutional services such as counselling sessions.

\section{Sample Size calculation}

The sample size calculation is based on the ability to detect a medium size or larger clinical effect. This corresponds to a standardized mean difference (Cohen's d) of $d$ > 0.45 [19]. We will test the hypotheses at a conventional power of ( 1 - beta) $=0.80$ and alpha $=0.05$, 2tailed (cf. Friedman et al., 1996). For this $n=78$ is required per condition, therefore a total of 156 participants. Finally, we want to compensate for possible dropout during the trial. The drop-out rate from the pilot study was $28 \%$ [9]. We therefore need to enrol $156 /(10.28)=214$ participants during the trial.

\section{Cost and outcome measurement}

\section{Effectiveness study}

Clinical outcomes will be measured using various questionnaires. The primary outcome of the clinical effect study is the symptoms, complaints and well-being of the young person measured with three questionnaires, each focussing on a different aspect of symptoms, complaints and well-being.

\section{Youth Self Report List (YSR) of the Children Behaviour Checklist}

The Youth Self Report List will be used to measure each subject's competencies and behavioural problems. The instrument is proven to be valid and has a good internal consistency $[20,21]$. The Youth Self Report List (YSR) is part of the Child Behaviour Checklist which is a questionnaire measuring internalizing and externalizing psychological problems in young people $[20,21]$. The Self-Report List has three versions: one each for parents, teachers and young people. For this study we use the young person's version. 


\section{Secondary clinical outcome}

The Effect Evaluation List (EEVL) is a Dutch questionnaire tailor-made for the online Kopstoring programme: it measures several aspects of coping skills and functioning. The instrument consists of 8 items in two main dimensions: biographical information about the respondent (19 items) and the effect of the COPMI intervention (61 Items). The EEVL has been proven to be valid and has good reliability [22].

\section{Quality of Life (EuroQol 5D)}

The EQ-5D is a self-administered questionnaire. Both generic quality of life, as well as utilities, will be derived by means of the EQ-5D, which will be completed by the young people. The EQ-5D has been chosen because it is a widely used quality of life instrument (nationally and internationally) and its reliability and validity is well established [23]. The EQ-5D contains 5 dimensions of health-related quality of life: mobility, selfcare, daily activities, pain/discomfort and depression/anxiety. Each dimension can be rated on three levels: no problems, some problems and major problems. In addition, the EQ-5D consists of a visual analogue scale (VAS) ranging from zero (worst imaginable health state) to 100 (best imaginable health state).

\section{Utilities}

Utilities will be derived from the EQ-5D using the following steps. The 5 dimensions will be summarized into a health state. Utility values will be calculated for these health states, using preferences elicited from a general population, the so-called algorithms. One algorithm has been established using a general population from the UK, the Dolan tariff [24]. For the sensitivity analysis the Dutch algorithm will be used [25]. The utility values derived will be used to compute Quality Adjusted Life Years (QALYs). A QALY combines survival and utilities. The utilities at the three time points will be used to compute the Quality Adjusted Life Years gained (QALY) using of the area under the curve method.

\section{Symptom Checklist (SCL-90)}

The Symptom Checklist has 90 items, each answered using 5 options covering 8 dimensions. These dimensions are: phobia, anxiety, depression, physical complaints, insufficient acting and thinking, distrust and interpersonal sensitivity, hostility and sleeping problems. The SCL-90 is a psychopathology-indicator and covers both somatic and psychological symptoms. It takes about 20 minutes to complete and has been tested and appears to be a valid instrument [26]. 


\section{Economic evaluation study}

In this economic evaluation we collect data on service use to derive the costs of using these services. Collecting data on service use:

Resource use questionnaire The use of the Kopstoring programme will be measured as well as the use of other resources both within and outside the healthcare sector. A questionnaire will be developed to measure the use of resources.

Productivity losses (PRODISQ) Production losses will be measured using the PROductivity losses and DISability Questionnaire (PRODISQ), a module-based questionnaire. This questionnaire will be used only for those with a paid job. Three modules will be used for this study to gather data on absenteeism and presenteeism. Production costs will be calculated by using the friction cost method $[27,28]$.

Productivity losses (PRODISQ) and School absenteeism For the young people who go to school, we adjusted the same three modules from the PRODISQ as we did to measure absentieesm and presenteeism among the working young persons.

\section{Process evaluation}

Qualitative Interviews Interviews will be conducted with the caregivers of the mental health services who provide the online Kopstoring programme. We will ask them questions about offering the programme as described in the protocol and about the process of enrolment. In addition to conducting these qualitative interviews with caregivers, we will send a short online questionnaire to the programme respondents.

Credibility/Expectations Questionnaire (CEQ) The credibility and expectations questionnaire, a 6-item list, is used to determine what the respondents expect from the online programme and how many credits they give the intervention.

Credibility and Expectations will be measured by the Credibility/Expectations Questionnaire (CEQ) which consists of 6 items using a 9- or sometimes 10-point Likert scale. This instrument is meant to measure the expectations and credibility a person has about the received intervention [29]. The questionnaire can be used at baseline and during follow-up to compute the different scores in expectation and credits one gives to a particular therapy. In their article Borkovec et al show that the instrument has proven to be valid and that the psychometric properties are of good quality [30]. It will take around five minutes to complete the 6 items of this instrument. 


\section{Analyses}

Data will be analysed according to the intention-to-treat principle, which means including data from all participants irregardless of whether they received the intervention or not. Missing data on the item level will be handled using SPSS missing values analysis. Completely missing measurements will be handled using multiple imputation. A baseline analysis will be performed to examine the comparability of groups at baseline for both costs and outcomes. Differences in baseline will be controlled using applied methods to correct these differences [31]. Losses to follow-up will be described.

The analysis consists of comparing the assessments and scores of the respondents on the questionnaires. Clinical outcomes after the intervention (8 weeks), at three months, six months and twelve months will be compared between groups and within groups, which means we want to compare the effect of the intervention in respondents in the intervention and control groups and assess the additional effects before and after receiving the intervention.

\section{Economic evaluation study}

The economic analysis will also be performed according to the intention-to-treat principle. Handling of missing data will be similar to the effectiveness study. A baseline analysis for the economic evaluation will also be performed to examine the comparability of groups at baseline for both costs and outcomes. If necessary methods will be applied to control for differences in baseline [31]. Despite the usual skewness in the distribution of costs, the arithmetic means are generally considered to be the most appropriate measures to describe cost data [32]. Therefore, arithmetic means (and standard deviations) will be presented. Non-parametric bootstrapping will be used to test for statistical differences in costs between the intervention and control group. Nonparametric bootstrapping is a method based on random sampling with replacement based on the participants' individual data [33]. The bootstrap replications will be used to calculate $95 \%$ confidence intervals around the costs $(95 \% \mathrm{Cl})$, based on the 2.5th and 97.5th percentiles.

The incremental cost-effectiveness ratio (ICER) will be determined on the basis of incremental costs and the effects of the Kopstoring programme in comparison with care as usual. The cost-effectiveness ratio will be stated in terms of costs per unit of outcome; the cost-utility ratio will focus on the incremental cost per QALY gained. The ICER will be calculated as follows. ICER $=(\mathrm{Ci}-\mathrm{Cc}) /(\mathrm{Ei}-\mathrm{Ec})$, where $\mathrm{Ci}$ is the annual total cost of the Kopstoring group, $\mathrm{Cc}$ is the annual total cost of the care as usual group, Ei is the effects at the 6-month follow-up for the Kopstoring group and Ec is the effect at the 6-month follow-up for the care as usual group. The robustness of the ICER will be checked by nonparametric bootstrapping. Bootstrap simulations will also be conducted in order to quantify the uncertainty around the ICER, yielding information about the 
joint distribution of cost and effect differences. The bootstrapped costeffectiveness ratios will be plotted subsequently in a cost effectiveness plane, in which the vertical line reflects the difference in costs and the horizontal line reflects the difference in effectiveness. The choice of treatment depends on the maximum amount of money that society is prepared to pay for a gain in effectiveness. Therefore, the bootstrapped ICERs will also be depicted in a cost-effectiveness acceptability curve, showing the probability that Kopstoring is cost-effective using a range of ceiling ratios. To demonstrate the robustness of our base-case findings a multi-way sensitivity analyses will be performed in which assumptions in the base case analysis will be recalculated to assess whether they have influenced the incremental cost-effectiveness ratio (ICER), for example by varying unit costs and volumes between minimum and maximum [33]. The bootstrap replications will be used to calculate $95 \%$ confidence intervals around the median costs, while the $95 \% \mathrm{Cl}$ will be based on the 2.5 th and 97.5 th percentiles.

\section{Process evaluation}

We will monitor whether certain aspects are going according to protocol and on schedule by qualitative interviews, data assessment and the use of the Credibility and Expectancy questionnaire. The analysis will consist of the following steps:

1. Analyzing the qualitative interviews

2. Comparing the results of the analysis with the standards and requirements in the protocol

3. Identifying factors where the delivery of the intervention deviates from protocol (factors that harm working according protocol) and factors that stimulate working according to protocol (success factors)

4. Analyzing the participants' expectations and beliefs in the effects of the intervention according the C\&E questionnaire.

\section{Collaboration}

The initiative for this study came from Maastricht University working together with Trimbos Institute. This study will be conducted with support from professionals from several disciplines. The London School of Economics will be involved in the academic part of the study. Mental health institutions in the Netherlands will be involved in the delivery of the online preventative intervention, including Dimence (Deventer), GGNet (Warnsveld), De Gelderse Roos (Wolfheze), GGNet Apeldoorn, Dimence Almelo, GGZ Oost Brabant, De Grote Rivieren and GGZ Zuid Holland. The Trimbos Institute will be responsible for developing a PR-strategy and will be involved with the recruitment of participants.

This study is registered in the Netherlands Trial Register, part of the Dutch Cochrane Centre (NTR1982). The ethical committee from Maastricht University Academic Hospital has given ethical approval for performing the experiment. 


\section{DISCUSSION}

The field of E-mental health and internet therapies is growing. Many new therapies have been developed, but few have been evaluated. A lot of them are preventative, so there will be no direct harm if effectiveness is not established. The implementation of evidence-based medicine means that the effectiveness and costs of new therapies and interventions should be established. This study will provide information about Einterventions in Dutch society in general, but will also yield specific information about a vulnerable group of young people in society. In the event that we can show effectiveness and cost-effectiveness, the aim will be to implement this intervention as standard care in the Netherlands.

\section{Methodological considerations}

Interventions in the field of COPMI-problems are relatively new and internet interventions have scarcely been evaluated. Internet-driven research has the advantage of being anonymous, but it can be hard to keep participants in the study because there is no face-to face contact with the participants. The participants in this study are 16-25 years old. When a minor is involved in scientific research, the informed consent of the parents as well as of the minor is needed according to Dutch Law (WMO art. 4). This requirement for extended consent might lead to the under representation of younger participants, as they might not want to inform their parents about participating in the study and therefore be unable to get their consent.

\section{Feasibility}

Feasibility was a very important key point in writing the proposal for the study. To establish effectiveness 214 respondents are needed. To ensure we could recruit sufficient young people we devised a PR-strategy including high quality advertisements. Links banners and other methods for reaching the target population, will be used. A special budget has been reserved for this purpose.

\section{CONCLUSION}

Recent developments show the pressing need for studying the effectiveness of interventions for children of parents with a mental illness or substance use disorder. In a meeting of the House of Representatives in May 2009, critical questions were asked about the growing prevalence of COPMI-children and the lack of good interventions [34]. While E-mental health interventions are likely to be easily accessed by young people, there is a pressing need to study the (cost)-effectiveness of these recent developments in health care. There is hardly any evidence, but what exists is promising [12]. This study builds on this evidence and provides a unique opportunity for develop- 
ing research techniques in this new area, as well as supporting the provision of better services for COPMI.

\section{Acknowledgements}

The study is financed by the ZonMw prevention programme. Extra funding is provided by the Innovatiefonds Zorgverzekeraars. 


\section{REFERENCES}

1. The forgotten child, EUFAMI. [http://eufami.org].

2. Association APA: Diagnostic and statistical manual of mental disorders, (DSM). 1994.

3. Factsheet Preventie 2: Kinderen van ouders met psychische problemen.[http://www.trimbos.nl].

4. Factsheet Online Groepcursus Kopstoring. [http://www.trimbos.nl].

5. Rutter M, Quinton D: Parental psychiatric disorders: effects on children. Psychological Medicine 1984, 14:853-880.

6. Fraser CE, Anderson K, Lloyd D, Judd F: Intervention programmes for children of parents with a mental illness: a critical review. International Journal of health Promotion 2006, 8:8-19.

7. Beardslee W, Gladstone T, Wright E, Cooper A: A Family-Based Approach to the Prevention of Depressive Symptoms in Children at Risk: Evidence of Parental and Child Change. Pediatrics 2003, 112:e119e131.

8. Beardslee W, Ellen M, Wright E, Salt P, Drezner K, Gladstone T, Versage E, Rothberg P: Examination of children's response to two preventive intervention strategies over time. Journal of the American Academy of Child and Adolescent psychiatry 1997, 36:196-204.

9. Veen C, van der Zanden R: Procesevaluatie Kopstoring. Utrecht: Trimbos Insituut 2007.

10. Beardslee WR, Keller MB, Lavori PW, Klerman GK, Dorer DJ, Samuelson H: Psychiatric disorder in adolescent offspring of parents with affective disorder in a non-referred sample. Journal of Affective Disorders 1988, 15(3):313-322.

11. Fact sheet I.COM. [http://www.trimbos.nl].

12. Kathenthaler E, Schackley P, Stevens K, Beverly C, Parry G, Chilcott J: A systematic review and economic evaluation of computerized cognitive behaviour therapy for depression and anxiety. Health Technology Assessment 2002, 6(22).

13. Marks IM, Cavanagh K, Gega L: Computer-aided Psychotherapy: Revolution or bubble? British Journal of Psychiatry 2001, 191:471-473.

14. Marks IM, Cavanagh K, Gega L: Hands-on help: computer-aided psychotherapy. New York: Psychology press 2007.

15. Brooks R, EuroQol Group: Euroqol: the current state of play. Health Policy 1996, 37:53-72.

16. The EuroQol Group: EuroQol - a new facility for the measurement of health-related quality of life. Health Policy 1990, 16(3):199-208.

17. WHO: Workbook 4 Process Evaluations. [http://www.unodc.org/docs/treatment//process_evalu ation.pdf].

18. Leunissen M, Rosenbrand R, Zanden van der AP: Online cursus Kopstoring Een preventief groepsaanbod via internet voor jongeren (16-25 jaar) van ouders met psychische en/of verslavingsproblematiek. Utrecht: Trimbos Institute 2007.

19. Lipsey MW, Wilson DB: The efficacy of psychological, educational, and behavioral treatment. American Psychologist 1993, 48:1181-1201.

20. Achenbach TM: Manual for the Child Behavior Checklist/4-18 and 1991 Profile. Burlington VUoV, Department of Psychiatry 1991.

21. Achenbach TM: Integrative Guide to the $1991 \mathrm{CBCL} / 4-18, \mathrm{YSR}$, and TRF Profiles. Burlington VUoV, Department of Psychology 1991.

22. Huijnen SEA, Valkenberg IMC: Handleiding bij de Effect Evaluatie Lijst voor KOPP/KVOpreventiegroepen voor adolescenten. Utrecht: Trimbos 2005.

23. Brooks R: The EuroQol: The current state of play. Health Policy 1996, 37(1):53-72.

24. Dolan P: Modeling valuations for EuroQol health states. Medical Care 1997, 35:1095-1108. 
25. Lamers LM, Stalmeier PFM, McDonnell J, Krabbe PFM, van Busschbach JJ: Kwaliteit van leven meten in economische evaluaties: het Nederlands EQ-5D-tarief. Nederlands Tijdschrift voor Geneeskunde 2005, 149:1574-1578.

26. Arrindell WA, Ettema JHM: Handleiding bij een multidemensionele psychopathologie-indicator. Lisse: Swets \& Zeitlinge 1986.

27. Koopmanschap M: PRODISQ: a modular questionnaire on productivity and disease for economic evaluation studies. Expert review of Pharmacoeconomics and Outcome research 2005, 5:23-28.

28. Koopmanschap M, Meerding W, Evers S, Severens J, Burdorf A, Brouwer W: Handleiding voor het gebruik van PRODISQ. Een modulaire vragenlijst over de relatie tussen ziekte en productiviteitskosten. Toepasbaar bij economische evaluaties van gezondheidszorgprogramma's voor patiënten en werknemers. Rotterdam/RMEU, Maastricht EMCU 2004.

29. Borkovec T, Nau S: Credibility and of analogue therapy rationales. Journal of Behaviour Therapy and Experimental Psychiatry 1972, 3:257-260.

30. Devilly GJ, Borkovec TD: Psychometric properties of the credibility/ expectancy questionnaire. Journal of Behavioral Therapy and Experimental Psychiatry 2000, 31:73-81.

31. Manca A, Hawkins N, Sculpher MJ: Estimating mean QALYs in trial based cost-effectiveness analysis: the importance of controlling for baseline utility. Health Economist 2005, 14(5):487-496.

32. Ramsey S, Willke R, Briggs AH, Brown R, Buxton M, Chawla A, Cook J, Glick H, Liljas B, Pettini D, et al: Good research for cost-effectiveness analasys alongside clinical trial: The ISPOR RCT-CEA Task Force report. Value in Health 2005, 8(5):821-833.

33. Briggs $\mathrm{AH}$, Wonderling $\mathrm{DE}$, Mooney CZ: Pulling cost-effectiveness analysis by bootstraps: a nonparametric approach to confidence interval estimation. Health Economist 1997, 6(4):327-340.

34. Ministerie van Volksgezondheid WeS: Kamervragen 18 mei 2009: Antwoorden op kamervragen van Bouwmeester en Bouchibiti over kinderen van ouders in de geestelijke gezondheidzorg (GGZ) en de forensische GGZ. Den Haag Ministerie van Volksgezonheid 2009. 



\section{CHAPTER 3}

\section{An online health prevention intervention for youth with addicted or mentally ill parents: \\ experiences and perspectives of participants and providers from a randomized controlled trial}

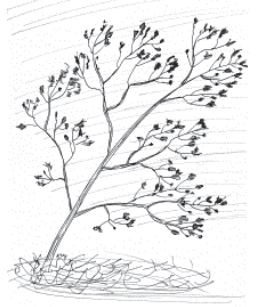

Woolderink M, Bindels J, Evers SM, Paulus AT, van Asselt AD, van Schayck CP (2015) An online health prevention intervention for youth with addicted or mentally ill parents: experiences and perspectives of participants and providers from a randomized controlled trial.

Journal of Medical Internet Research; 2015; Dec 2;17 (12) Doi: 10.2196/jmir. 4817. 


\section{ABSTRACT}

Background: Mental illnesses affect many people around the world, either directly or indirectly. Families of persons suffering from mental illness or addiction suffer too, especially their children. In the Netherlands, 864,000 parents meet the diagnostic criteria for a mental illness or addiction. Evidence shows that offspring of mentally ill or addicted parents are at risk for developing mental disorders or illnesses themselves. The Kopstoring course is an online 8-week group course with supervision by 2 trained psychologists or social workers, aimed to prevent behavioral and psychological problems for children (aged 16 to 25 years) of parents with mental health problems or addictions. The course addresses themes such as roles in the family and mastery skills. An online randomized controlled trial (RCT) was conducted to assess the effectiveness of the Kopstoring course.

Objective: The aim was to gain knowledge about expectations, experiences, and perspectives of participants and providers of the online Kopstoring course.

Methods: A process evaluation was performed to evaluate the online delivery of Kopstoring and the experiences and perspectives of participants and providers of Kopstoring. Interviews were performed with members from both groups. Participants were drawn from a sample from the Kopstoring RCT.

Results: Thirteen participants and 4 providers were interviewed. Five main themes emerged from these interviews: background, the requirements for the intervention, experience with the intervention, technical aspects, and research aspects. Overall, participants and providers found the intervention to be valuable because it was online; therefore, protecting their anonymity was considered a key component. Most barriers existed in the technical sphere. Additional barriers existed with conducting the RCT, namely gathering informed consent and gathering parental consent in the case of minors.

Conclusions: This study provides valuable insight into participants'and providers'experiences and expectations with the online preventive intervention Kopstoring. It also sheds light on the process of the online provision of Kopstoring and the accompanying RCT. The findings of this study may partly explain dropout rates when delivering online interventions. The change in the (financial) structure of the youth mental health care system in the Netherlands has financial implications for the delivery of prevention programs for youth. Lastly, there are few RCTs that assess the effectiveness and cost-effectiveness of online prevention programs in the field of (youth) mental health care and not many process evaluations of these programs exist. This hampers a good comparison between online interventions and the expectations and experiences of the participants and providers. 


\section{INTRODUCTION}

Mental illnesses affect many people around the world, either directly or through another person. The prevalence of mental illnesses, such as depression, is high [1] and many among those who suffer from mental illness or addiction are parents. The families of persons suffering from mental illness experience a degree of burden too $[2,3]$. Results of a Canadian survey show that 1 in every 10 children lives with a parent with a psychiatric disorder and 1 in every 6 children lives in a household with at least 1 person affected by a psychiatric disorder [4]. In these situations, mental illness is not only a problem for the patients, but also for their family and their children in particular. Various studies report that offspring of mentally ill/addicted parents are regarded at risk for developing mental illness (eg, depression and anxiety disorders) themselves [5-8].

Although preventive interventions for children of parents with mental illness or addiction are scarce, some interventions have been developed for this vulnerable group $[9,10]$. Most of these interventions are intended to be performed face-to-face and only a few are developed for online use. However, given the nature of the target group (eg, including minors, being at risk) and the problem being addressed, a face-to-face intervention is associated with numerous challenges regarding recruitment and inclusion. National Dutch data show that with current face-to-face interventions, the target population was not reached sufficiently and information did not find its way to the population [11]. The youth that were reached valued their anonymity and privacy, which makes face-to-face interventions less appealing. Therefore, online interventions seem to be a worthy alternative.

In the Netherlands, 864,000 parents meet the diagnostic criteria of a mental illness or addiction [12,13]. An online preventive course (Kopstoring) for children of these parents was developed. Kopstoring is one of the few online interventions for children of parents with mental illness or addiction disorders. The course is based on evidencebased theories and a face-to-face course developed for the same population. The Dutch Kopstoring course is designed for adolescents from ages 16 to 25 years. A pilot study assessing the effects of the course showed Kopstoring to be effective in improving participants' coping and mastery mechanisms [14].

The objective of this study is to gain knowledge about expectations, experiences, and perspectives of participants and providers of the online Kopstoring course. The research questions were how was the process of the delivery of the online Kopstoring course perceived by Kopstoring participants and providers and what were their expectations and experiences with this course? 


\section{METHODS}

A process evaluation was performed to evaluate the online delivery of Kopstoring and the experiences and perspectives of Kopstoring participants and Kopstoring providers. Interviews were performed with both groups. This section first describes the Kopstoring course and the accompanying randomized controlled trial (RCT; trial registration: NTR1982 [15] and subsequently the methods used in this process evaluation.

\section{Intervention}

The Kopstoring course aimed to prevent behavioral and psychological problems in offspring at risk and was offered to adolescents from ages 16 to 25 years. The Kopstoring course was an online 8-week group course with supervision by 2 trained psychologists or social workers from a participating mental health institution in the Netherlands. Every week a different theme was discussed and participants were expected to prepare for the weekly meetings by doing homework. The course had a preventive nature; therefore, adolescents were screened to ensure that they were not diagnosed with an illness as classified by the Diagnostic and Statistical Manual of Mental Disorders, Fourth Edition (DSM-IV) diagnoses. Screening was executed by the mental health institutions. In addition, participants needed to have access to a computer with an Internet connection and be able to participate weekly.

Alongside the process evaluation described in this paper, a RCT was conducted. The aim of the RCT was to examine the effectiveness and cost-effectiveness of the Kopstoring course. Participants were randomly allocated either to immediate enrollment in the Kopstoring course (intervention group) or enrollment after a 6-month waiting list (control group). Because the course was completely digitalized, the recruitment was done mainly, but not exclusively, through online recruitment, including banners, Facebook advertisements, links to the website, etc. In addition, articles were published in national and regional magazines and newspapers, school visits were performed, and an interview was broadcasted on a radio station.

At the start of the project, 9 Dutch mental health institutions participated and each institution trained 2 professionals for the provision of the Kopstoring course. All planned Kopstoring courses provided during this study were provided by 7 professionals from 4 different mental health institutions. These providers were invited to participate in interviews. In total, 4 providers agreed to participate in an interview.

The providers of the Kopstoring course were all female and approximately 30 years of age; all had a Master's degree and had experience working in this field for 5 to 7 years. 


\section{Sample}

In this process evaluation, 2 groups were included: participants $(n=13)$ and providers $(n=4)$ of the Kopstoring course.

The Kopstoring participants were selected from both the intervention and the control group of the RCT. Participants received an email in which they were invited to be interviewed.

To select participants for the interviews from the trial pool, a maximum variation strategy was used to gather information from a sample with as much variation as possible to collect as many different perspectives [16]. This was done by looking at several characteristics (eg, trial arm, age, sex, online and written consent for the trial, dropout). See Table 1 for the characteristics of the Kopstoring participants who were interviewed. The interview sample is fairly comparable to the RCT sample.

Table 1: Characteristics of interview participants versus trial participants

\begin{tabular}{lll}
\hline Characteristics & Interview participants, $\mathrm{n}(\%) \mathrm{n}=13$ & Trial sample, $\mathrm{n}(\%) \mathrm{n}=104$ \\
\hline Age $^{\mathrm{a}}$ (years) & $3(23)$ & $20(19.2)$ \\
$16-17$ & $10(77)$ & $84(80.7)$ \\
$>18$ & & \\
Sex & $12(92)$ & $93(89.4)$ \\
$\quad$ Female & $1(8)$ & $11(10.6)$ \\
$\quad$ Male & $8(62)$ & $55(52.9)$ \\
Treatment group & $5(38)$ & $49(47.1)$ \\
Intervention & & \\
Waiting list control & $11(85)$ & $97(93.3)$ \\
Adherence to Kopstoring course & & $7(6.7)$ \\
Completed & $2(15)$ & \\
Started but did not finish &
\end{tabular}

\footnotetext{
${ }^{\text {a }}$ Age at time of registration for the course

${ }^{b}$ To this point, data were checked up until 6 months after registration due to the pending follow-up assessments
} 


\section{Data Collection}

Data were collected through semistructured individual interviews with a list of topics to be discussed (Textbox 1). Interviews were held between November 2014 and February 2015. The topic list was made by the research team in collaboration with the national coordinator of Kopstoring and the team of course providers.

Textbox 1: Interview topic list for participants

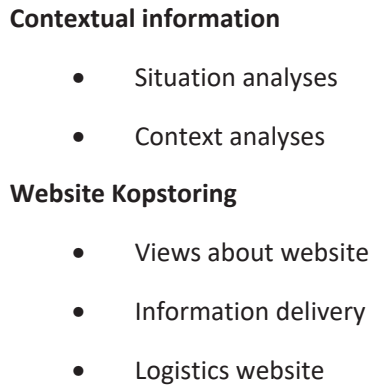

- Situation analyses

- $\quad$ Context analyses

Website Kopstoring

- Views about website

- Information delivery

- $\quad$ Logistics website

Effects of the course

- Views about the course

- $\quad$ Anticipated effects

- $\quad$ Experienced effects before, during, and after the course

- Barriers and success factors for completing the course

Process and content-related aspects of the course

- Components (themes) of the course

- Tailor-made health care

- Technical aspects of delivery online

Research

- Understanding study aspect

- Motivation

- Experience

Anonymity was very important for offspring of parents with mental illness or addiction problems. Therefore, interviews were conducted over the phone. Participants decided the time of the interview so they could be sure they were able to talk freely. Interviews with the providers were also conducted over the phone, but due to time constraints 
not because of anonymity. Interviews were held in Dutch. Textbox 2 displays the topic list for the providers.

\section{Analysis}

The interviews were audiotaped and transcribed and identifiable information was removed to ensure anonymity. The interviews were analyzed by using inductive qualitative content analysis, specifically conventional content analyses [17]. This method helped provide an in-depth understanding about underlying perspectives and qualitative methods are inductive and reflexive and it allowed the use of quotes [18]. As a first step, the interviews were read by 2 researchers separately to identify emerging themes and subthemes and then labels were attached to the parts related to these themes. Secondly, new themes were added to existing themes and labeled accordingly. After the 2 researchers reached consensus, the interview data were clustered into themes and subthemes. Finally, citations of the interviewees were identified per theme and visualized in a data matrix. After approximately 13 interviews, no new information emerged from the interviews with the participants.

\section{RESULTS}

Five main themes emerged from the interviews: (1) background, (2) the requirements for the intervention, (3) experience with the intervention, (4) technical aspects, and (5) research aspects. In this section, each theme and its subthemes will be discussed from the participants' and the providers' perspectives.

\section{Background}

For the participants, the background mainly related to the motivation and reason for participation, the route to registration, and expectations of the online course. The providers' background related to their experiences with the provision of similar faceto-face courses and online interventions. 
Textbox 2: Interview topic list for providers

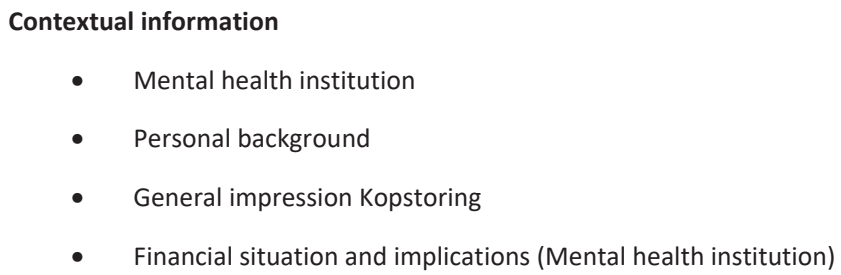

- Mental health institution

- Personal background

- $\quad$ General impression Kopstoring

- Financial situation and implications (Mental health institution)

Website Kopstoring

- Views about website

- Information delivery

- $\quad$ Logistics website

Provision, process, and content of the course

- Views about the content of course

- Advantages and disadvantages of online delivery

- $\quad$ Experiences with provision of Kopstoring

- $\quad$ Process from registration to allocation in group

- Barriers and success factors for provision of the course

- Technical aspects of delivery online

Research

- Motivation to participate

- $\quad$ Experience with the study

\section{Participants}

Participants from the Kopstoring course generally had 2 routes to arrive at the point of registration. Analyses showed that it was either a slow, lingering process in which the person already had the intention to change the situation for some time and was looking for a suitable way to address their needs or there was an acute situation which forced them to seek help right away. The following is an example of an acute situation that led to an immediate online registration:

"There was a real occasion leading to why I registered. It was September last year and my mother had a psychosis... and she attacked me that night." [Participant 12] 
A respondent for whom the situation was ongoing long before registration explained:

"I had a difficult time dealing with the situation and with the fact that my brother was placed into care (out of house placement). Well, I really could not handle it well, so they advised me to register for the course." [Participant 4]

There was no difference in results reported by participants who registered under pressure of an acute event or those who took their time to register for the course.

For both situations, there appeared to be several facilitators, for example, a psychologist, school mental health worker, or a family member pointing out the online course or participants who found the course through an Internet search. Despite the different problems and family situations of participants (eg, one person had an addicted mom, another had a mentally ill father, and both parents were mentally ill for a third person), the consequences, questions, and problems they were confronted with were very similar.

Motivations to participate could be divided into 4 categories: (1) sharing experiences with persons in the same situation, (2) learning how to cope with ill parents, (3) learning how to cope with their own problems, and (4) learning about mental illness or addiction:

"I hope it will be comforting for me to talk about my experiences with peers who went through the same experience." [Participant 11]

Most of the time, problems were not discussed with family members and friends. This explained their need for sharing with others who had been through the same experience. One participant explained this as:

"In a certain way, it provoked a sense of relief learning that other people were actually going through the exact same experience." [Participant 4]

All participants had easy access to the website and experienced no problems with the registration process. During this process, all prospective participants were asked about their expectations of the online course. The answers were concise for the most part and participants had clear expectations regarding the content and the anticipated effect of the course.

When asked about their goals, participants reported they expected to learn and understand more about their parent's illness or addiction, to learn how to cope with the illness or addiction, and to learn how to improve the situation at home and decrease problems themselves. 
One respondent explained that she hoped to find out how the situation got this "extreme" and to learn to deal with her mom so that they could improve their relationship and the situation. In accordance, other participants explained:

"I expect this to be very helpful, mainly that I have more understanding about the cause of the symptoms and how to deal better with my mother. I hope to learn ways and also to detach myself from my mother." [Participant 10]

Prospective participants, aged 16 to 25 years, had well-defined expectations of the online delivery and effects of the Kopstoring course.

\section{Providers}

Providers' general impression of the online delivery of Kopstoring was positive:

"It is just a very good program that does not require any change. That is, of course, very important." [Provider 1]

In some cases, the Kopstoring manual was considered theoretically so well written that providers used the same manual for face-to-face groups for children of mentally ill or addicted parents:

"Yes, I find that very good [the course manual/protocol]. I even use it as the manual in the face-to-face Kop-groups. This is because I consider it to be a very pleasant way how subjects are being discussed, which themes will be covered, like cognitive behavior therapy." [Provider 3]

\section{Requirements}

There are some requirements when providing online interventions. Firstly, participants and providers needed a computer connected to the Internet, log-in codes, and some privacy. For the providers, a budget was necessary to provide the Kopstoring course. Some barriers were encountered at the organizational level and regarding the financial structure.

\section{Participants}

In general, there were no barriers encountered to meet the requirements; however, one of the participants mentioned that when moving house she did not have access to the Internet, which made it impossible to log on to that session.

A second person explained that it was not always easy to find enough privacy in the house because there were always people around who did not know he was participating in the course: 
"The only thing that was difficult was finding a place to separate myself from others, and just having a moment for myself. That was difficult." [Participant 12]

\section{Providers}

Providers needed support from their managers within the mental health institution and adequate finances to provide the online course because online mental health interventions are not paid from public funds. In addition, providers of online anonymous interventions face the situation that costs will not be reimbursed by insurers due to the fact they cannot provide social security numbers or other personalized details. It is up to the management of a mental health institution to decide whether or not it is feasible to add Kopstoring in their portfolio. In addition, the financial situation and structure of mental health care divisions for minors (up to age 18 years) changed during the course of the RCT. In short, municipalities became responsible for the policy and execution of budgeting of prevention interventions in youth mental health care. This shift had tremendous consequences for the delivery of mental health care interventions for youth up to age 18 years. In some institutions, prevention and youth departments were declared redundant and, consequently, the institutions withdrew their consent to provide the Kopstoring course. There were many problems encountered with finding funding to provide the Kopstoring course. One provider explained:

"Health insurers are not paying for delivery of Kopstoring because it is provided anonymously and a health insurer only wants to reimburse when they have all details from the client. So that means you have to provide them with a health insurer registration number and social security number, everything, and we do not ask these details when providing Kopstoring because we want it to be anonymous. So the only remaining source is the municipality and...naturally the municipality actually only wants to pay for inhabitants of that municipality." [Provider 1]

Initially, the costs of the courses were reimbursed by additional funding obtained by the research team. This meant that when the study period ended, reimbursement of the courses also came to an end. One provider explained that once the research team did not fund provision of the courses anymore, their mental health institution stopped providing the Kopstoring course.

\section{Experiences with the Course}

\section{Participants}

Participants described many different effects on their daily life and their problems. The first and most emphasized effect of the course was peer contact. Speaking with youth 
in the same situation made participants feel less alone, relieved, and less guilty in some cases. The recognition of situations, problems, and decisions became something they could share with peers:

"My friends did not understand me. I have tried to explain, but then they would just say "awhhh it will be fine" and that was so nice to... with peers who might have a slightly different situation maybe, but that they also felt lonely and that you...share the same and have compassion for one another." [Participant 5]

A second component that was considered very effective was the psychoeducative part of the course. Learning about the illness or addiction of the parent gave insight into the behavior of the parent:

"I noticed that I experienced more peace with the fact that she has a drinking problem. It is the way it is and that will not change anymore." [Participant 3]

Furthermore, the participants learned tips and tricks on how to cope with the behavior and problem of the parent, which led to accepting the parent's problem and more peace in the family in some cases:

"Yes, I argued with my father pretty often if he had something going on. They said I should actually try to reduce these moments and I am able to do that now." [Participant 1]

Participants also reflected on the content of the Kopstoring course and all themes were deemed important for the course to be effective. Almost all interviewees pointed out that the "rate your week" component, which kicked off every session, was extremely valuable to them. "Rate your week" was a simple but effective way to share experiences about the past week and a platform for questions and peer contact. $A$ respondent explained the working mechanism of "rate your week":

"Rate your week was very interesting for me. For your self-reflection on whether or not the week went well and that you were able to look back later to see how it was in the beginning of the course and how am I doing now? I really liked that; what are the positive things that make you also feel very positive instead of focusing on the negative things." [Participant 9]

However, one of the sessions in which the educational component was key was mentioned to be a bit repetitive.

Experiences with the online program also translated into barriers. Barriers that existed were lack of time to discuss the homework assignments, some participants mentioned 
the course focused too much on the younger participants (students), and a couple of participants mentioned the homework was complicated.

Facilitating factors to adhere to the intervention and study were also mentioned. The online delivery of the intervention was mentioned as a major facilitating factor to start and finish the course. Firstly, online delivery was found to be convenient and ideal for participation in a safe and self-chosen environment. Secondly, online delivery protected the anonymity and privacy of the participant, which encouraged the participants to be more open:

"Openness, yes...because it is online you do not have the feeling that everyone was looking at you. Then you can just write and maybe if you had to cry or so...no one was able to see that." [Participant 8]

An often-mentioned stimulating factor was the attitude of the provider. Most of them were easy to access and always available (by email) to answer questions and monitor the participant:

"I noticed that whenever the trainer tells us that she is still available to answer questions after the end of the course, or emails or these kind of things, that felt incredibly nice, that someone is still there who takes time, yeah where you can lean on. So that I consider to be very pleasant." [Participant 6]

Only one participant mentioned that the attitude of the provider was not meeting her expectations. This participant stopped participating in the course after session 3 and was not included in the RCT.

\section{Providers}

All providers were satisfied with the content and agreed that all the important concepts were covered. The most important aspect was considered to be the online delivery, which ensured anonymity for the participant:

"Within the Kopstoring course, they [participants] can, of course, tell their story very anonymously. Nobody knows that you participate in the group and what is bothering you. That is a huge advantage; that it becomes easily accessible for youngsters, but that they nevertheless can benefit and become more aware of what is going on and get answers to their questions." [Provider 2]

For the content, the most important part was considered to be the exchange of experiences during "rate your week" at the beginning of each session. 
All providers were asked several questions to check adherence to the protocol. They indicated they followed protocol except for one rule: the protocol described delivery of the course should be done by 2 professionals together. In practice, all providers delivered the course individually due to cost reductions. This was, however, not considered to be a barrier because providing the course for a group of up to 6 participants was highly manageable for one provider. There were some (technical) barriers experienced for the online delivery, but for providers the main barriers were experienced in the financial administrative field.

Positive factors were described as the feasibility of the online delivery and the possibility to deliver the course from home, the interactive group process, and the growing number of participants:

"That every time again I am so surprised how close a group can become online and that as quick as in the first session they are already so open. And that is due to the anonymity that participants are just so open and what they think or experience...Yes, I think that this is very special and that stimulates me to provide the course over and over again and just getting back from them that they appreciate being heard." [Provider 1]

\section{Technical Aspects}

The technical component was found to be extremely important by both the participants and providers of the Kopstoring course. Not only were the technical aspects (eg, the website or the chat box) considered positive factors, the same technical aspects were mentioned as barriers for participating in or providing the course. Almost every interviewee mentioned technical problems of some degree (from having a slow system to being thrown out of the chat box) and providers also mentioned these disturbances interrupting the courses. However, there were no major incidents mentioned that fully hindered participation in the long run. 


\section{Participants and Providers}

The delivery of Kopstoring online was considered very positive; however, it seemed to also cause problems. Online delivery can be a double-edged sword; the convenience of the online aspect can be a pitfall at the same time.

Technical aspects were the design of the website and the chat box. Both were considered well designed and suitable for the target population. The website was described as complete, clear, colorful, and cheerful, which for participants is important. Providers shared this opinion about the website:

"Just by clicking on the website, I consider it quite clear...I think it is convenient when registering for the course that the data when courses are starting is visible." [Provider 2]

"I considered it [chat box] very well done that different persons were indicated with different colors, so you were able to see who...to increase visibility." [Participant 3]

\section{Barriers}

The technical problems described by both groups ranged from technical hiccups to some more prominent problems, such as not being able to log on to group sessions or being locked out by the system.

Minor technical problems involved a slow system, not being able to see when someone was typing, unable to see homework assignments on the screen, and double messaging occurring. These problems were mentioned, but were interpreted as minor problems and a consequence of an online working environment:

"Sometimes it took like a minute or so before the text would be displayed or then it got stuck or we were removed from the chat box. Yeah every now and then we would struggle a bit." [Participant 12]

Providers also described experiencing the same minor problems. The technical platform and responsibility related to the technical aspects were more numerous for the providers. They were responsible for all requirements to be met even before groups started online sessions. 


\section{Research Aspects}

Participants were confronted with aspects such as a 6-month waiting list, randomization, extended follow-up, and questionnaires. It appeared that most participants understood there was a study linked to the Kopstoring course, but none of them could describe what the consequences were for them; regardless, patient information sheets were given to them by email, mail, and online:

"I did know there were more groups where you could be allocated to, but I did not know that there was a chance you would have to wait half a year." [Participant 12]

Participants who were allocated to the waiting list believed they had to wait because the group was full. This explained why most participants expressed no strong negative experiences toward the research components. In some cases, the waiting list was experienced as problematic, although most participants accepted the waiting period:

"Yes, there was one group and I was hoping I could start right away, but unfortunately no. I had to wait half a year. That was really annoying. I needed help at that particular moment." [Participant 8]

Most of the participants indicated altruism as the main reason for participating in the study, although others participated because "it is part of the course":

"I just hope that there are more young adults who get this opportunity to participate in this kind of course...that here is research, because yeah I feel that there is too little for Kopp? (children of mentally ill or addicted parents, for those groups)." [Participant 9]

Participants provided feedback on the length of the questionnaires and some technical problems related to not being able to open links or links expiring due to waiting too long to fill out the questionnaire. From the interviewed Kopstoring participants, 2 persons had incomplete data; when asked why, there was no specific reason, but they said they forgot. In addition, some of the respondents mentioned the phrasing of some of the questions. They disliked the questions because they were too focused on the younger participants (students) living with their parent(s).

During the interviews, a couple reasons for the poor response rates were mentioned: laziness or forgetfulness and problems with parental informed consent in case of a minor.

Participants were asked to give online consent and written consent sent by post. Only one participant did not send back the informed consent papers and, therefore, was not 
a participant in the RCT. She explained that she forgot to send the forms back, whereas the other participants had clear motivations for participation in the study. Some minors sent back their informed consent papers, but not those of their parents, stating they did not wish their parents to know let alone sign a consent paper for participation in the study. Some minors found a way to let their parents sign.

\section{Providers}

For providers, the research aspects were proper barriers. The back office and technical interface changed so much with the migration to the study environment that the coordinator was forced to invest time into teaching herself the new system. Providers also pointed out that the inclusion of participants in the study would have been smoother if the research team had listened to their advice concerning some of the requirements for study inclusion:

"What I consider to be a real pity is that not enough weight is given to the advice of the professionals from the mental health institutions. ...Yeah, youngsters score incredibly high...if you are shocked by that as a researcher...It doesn't surprise or shock me anymore because I know that they score high, and I think it is very important to just reach out to these youngsters for participation in Kopstoring and motivate them to seek help." [Provider 1]

One other barrier mentioned was the frustration they had to deal with when a person was allocated to the waiting list control group. Providers explained, "It is the way it is when doing research but it remained sad you had to disappoint a person who needs help right there and right then."

Every 3 weeks, a new group was started. In addition, due to the allocation of participants to the waiting list, the number of people in the groups was reduced. Starting with smaller groups was considered a disadvantage because it was difficult to reach the full potential of the course.

Regardless of these barriers, providers were determined to reach the target number of participants for inclusion in the RCT. Their motivation was based on several stimulating factors. One provider explained that her opinion about the value of the product made her enthusiastic to provide the course and help with inclusion in the study: 
"I think you are enthusiastic if you see the value of research. That will lead to results and, surely, I hope the results are good. You also notice that participants have very positive experiences and so you feel you are working/providing a good product, so I think it matters a lot and the fact that it is studied, I only cheer for that." [Provider 4]

Also, the interest and engagement of the researcher played a role in the delivery of the Kopstoring course and the willingness to help:

"I enjoyed that you (researcher) were present at all meetings and gave an update on how the situation was and, yes, then we had an idea of what the situation was and that is what you are working towards." [Provider 2]

Providers explained there were barriers; despite these, they were able to work within the study parameters.

\section{DISCUSSION}

\section{Principal Results}

To our knowledge, this study is the first evaluation of the experience of providers and participants with an online-delivered prevention course for offspring at risk. Therefore, this study differentiates itself from existing international literature and provides new information. The few process evaluations performed to assess experiences with online programs focus on online treatment, programs for somatic diseases, or and/or an adult patient population. The findings of this study give insight into the experiences of participants and providers of an online prevention course called Kopstoring. It sheds some light on experience with as well as barriers and facilitating factors of online delivery. It elaborates on the expectations and experiences of both participants and providers. Analyses showed similar experiences for the 2 groups despite their different perspectives.

The main lesson learned from participants lies in their assessment of the course content and the barriers and facilitating factors for participating and adhering to an online course. The online aspect and anonymity proved to be important as well as their autonomy to decide to participate without interference from anyone else. In the Netherlands and some other countries, minors (participants younger than 18 years) need to provide the research team with written consent and their parent's consent for participating in a scientific study. This ignores the fact that minors can receive treatment (which is being assessed) of any kind from the age of 16 years without parental consent. Youth are considered capable of making an informed decision about treatment; however, for a scientific study we doubt their ability to make an independent and informed choice [19]. This subject was brought up even by participants older than 18 
years who said that if they had to provide parental consent, they would probably not have participated. They explained that we cannot expect them to ask their parents for consent when they are the root of their problems. There is a strong need to rethink the policy concerning consent in the case of interventions for vulnerable populations and interventions with a high level of anonymity (mostly online interventions). This statement endorses the debate in the literature questioning when a minor should be considered capable to give informed consent and therefore protect his/her anonymity $[20,21]$. Despite the differences in consent procedure for minors, no substantial differences between minors and participants older than 18 years are reported in this study.

The lesson learned from the providers of the Kopstoring course lies partly in their professional assessment of the content of the course, but mainly in the experience with providing online courses and the barriers and facilitating factors to provide the Kopstoring course. Analyses showed that providers of online interventions in RCTs might feel ignored and may experience a gap between the research team and providers, even though the provider has many years of experience with providing online interventions in this target population. This implies that there is a need for closer collaboration with providers, and perhaps even with the target population, when designing such interventions and accompanying studies [22,23]. Collaborating with stakeholders could have led to other research questions, methods, and the use of other questionnaires more suitable for the target population.

The current situation in the Netherlands for children in need of mental health care is unsettling. This study could not have taken place in a more inconvenient time and political setting than it actually did. In the same period the RCT was running, political decisions forced youth mental health care out of the hands of mental health institutions and made it subsequently a part of the local municipalities. Even if the results of cost-effectiveness studies, such as the Kopstoring RCT, show positive results, online interventions may not be provided due to the complex financial structure and lack of responsible bodies to finance online interventions. This also shows that implementation and implementation research in the Netherlands, but very likely in other similar countries, is nearly impossible for these types of intervention.

\section{Limitations of the Study}

There are several factors that could be considered to influence the findings of this study. The first is the number of interviews performed. One can question whether the small number of providers interviewed is sufficient to provide a complete overview of the ongoing issues. However, we do believe that providers who cooperated gave a lot of information about the Kopstoring course and the delivery of the course. We remained with only 9 providers who provided more than one course and were totally informed about every research detail. Therefore, we believe 4 providers were a good reflection of the 9 remaining providers and the group appeared to be homogeneous. 
For the participants, there is a different reason for the low response rate (42 people were invited, 17 responded, and 13 were eventually interviewed; response rate: $13 / 42,31 \%$ ) for participating in this study. The target population appears to be extremely difficult to reach. As shown in the analyses, they wish their anonymity to be respected and feel "safe" in an online environment and not face-to-face or on the phone. In addition, a feeling of shame and guilt regarding their problems blocks them from sharing their experiences with a researcher. Despite this, the majority of the participants were enthusiastic about the online prevention course and potential bias might occur with this. It is possible that youth with negative experiences with the course or research were not willing to be interviewed. Additionally, due to the sensitivity of this problem and the fact that the parents are involved, youth might find it difficult to speak about this with a third party (ie, might feel like "airing their dirty laundry" in public). However, for both participants and providers, repetition in the interviews showed a level of saturation.

A second limitation relates to the generalizability of the findings. It is noticeable that an overwhelming majority of Kopstoring participants, participating in the underlying RCT and this process evaluation, were female. This is probably not a good reflection of an open population, assuming there are an almost equal number of boys who have a mentally ill parent as there are girls. This leaves questions about generalizability unanswered. In addition, questions have been raised such as "are the findings useful in a similar online context, but with a different underlying intervention?" and "are the findings the same when comparing the online course to a similar face-to-face group?" It appeared that several factors added up; the online aspect, age, anonymity, and sensitive problems and anonymity lead to barriers doing research within this vulnerable group. The results of this study focus on youth with parents with mental illness or addiction problems. Despite these factors, some general elements can be identified that are useful in other online settings, such as the aspect of anonymity, consent, and practical issues.

\section{CONCLUSIONS}

Online support for offspring of parents with mental illness or addiction problems is considered effective by the participants. There are not many RCTs performed to assess the effectiveness and cost-effectiveness of online prevention programs in the field of mental health care [24]. Consequently, there are not many process evaluations of these online prevention programs performed. This hampers comparison between online programs and process of delivery and expectations. In addition, a face-to-face group is set up differently in structure and has fewer participants; therefore, it is difficult to use it in comparison to an online program. In this respect, this study is unique and sheds some light on experiences and barriers for online provision of a prevention course in the field of mental health care. 
The barriers for online provision of this health intervention are minimal, but the ones that exist lie in the technical sphere. Barriers for online research are multiple and touch on different aspects, such as informed consent, anonymity, lack of time, or just lack of interest. The findings of this study may explain partly why there are substantial dropout rates when delivering online interventions. The experiences of participants and providers of the Kopstoring course give valuable insights into the process of the online provision and study of Kopstoring.

\section{Acknowledgements}

The authors would like to thank all participants from the Kopstoring RCT and the ones who were willing to be interviewed for the process evaluation. In addition, the authors would like to thank Karlijn Arntz who has done an outstanding job as coordinator of the participating mental health institutions. We would also like to thank the participating mental health insitutions: Eleos, Dimence, Mindfit, GGNet, Vincent van Gogh Instelling \& Riagg Zuid, and Ypse Dixet for providing the Kopstoring course. This study was an addition to the Kopstoring RCT which is financed by ZonMw (The Netherlands Organisation for Health Research and Development grant number: 200210002) and provision of the Kopstoring course was funded by the Dutch Innovatiefonds Zorgverzekeraars. 


\section{REFERENCES}

1. Ustün T, Ayuso-Mateos J, Chatterji S, Mathers C, Murray CJ. Global burden of depressive disorders in the year 2000. Br J Psychiatry 2004 May;184:386-392

2. Maurin J, Boyd C. Burden of mental illness on the family: a critical review. Arch Psychiatr Nurs 1990;4(2):99-107.

3. Bijl R, Ravelli A, van Zessen G. Prevalence of psychiatric disorder in the general population: results of The Netherlands Mental Health Survey and Incidence Study (NEMESIS). Soc Psychiatry Psychiatr Epidemiol 1998;33(12):587-595.

4. Bassani DG, Padoin CV, Philipp D, Veldhuizen S. Estimating the number of children exposed to parental psychiatric disorders through a national health survey. Child Adolesc Psychiatry Ment Health 2009;3(1):6.

5. Beardslee WR, Gladstone TR, O'Connor EE. Transmission and prevention of mood disorders among children of affectively ill parents: a review. J Am Acad Child Adolesc Psychiatry 2011 Nov;50 (11):10981109. [doi: 10.1016/j.jaac.2011.07.020]

6. Weissman M, Wickramaratne P, Nomura Y, Warner V, Pilowsky D, Verdeli H. Offspring of depressed parents 20 years later. Am J Psychiatry 2006;163(6):20-28.

7. Weissman M, Fendrich M, Warner V, Wickramaratne P. Incidence of psychiatric disorder in offspring at high and low risk for depression. J Am Acad Child Adolesc Psychiatry 1992 Jul;31(4):640-648. [doi: 10.1097/00004583-199207000-00010]

8. Downey GJ, Coyne JC. Children of depressed parents: an integrative review. Psychol Bull 1990;108(1):50-76.

9. Siegenthaler E, Munder T, Egger M. Effect of preventive interventions in mentally ill parents on the mental health of the offspring: systematic review and meta-analysis. J Am Acad Child Adolesc Psychiatry 2012 Jan;51(1):8-17.e8. [doi: 10.1016/j.jaac.2011.10.018]

10. Fraser C, James E, Anderson K, Lloyd DF, Judd F. Intervention programs for children of parents with a mental illness: a critical review. International Journal of Mental Health Promotion 2006 Feb;8(1):9-20. [doi: 10.1080/14623730.2006.9721897]

11. De Jonge M, Ruiter M. Aard en Omvang GGZ- en Verslavingspreventie 2008- Ontwikkelingen in de inhoud, organisatie en omvang van de GGZ- en verslavingspreventie. Netherlands: Trimbos Instituut; 2010.

12. van Dorsselaer S, Zeijl E, van den Eeckhout S, ter Bogt T, Vollebergh W. HBSC 2005: Gezondheid en welzijn van jongeren in Nederland. Utrecht: Trimbos Instituut; 2007.

13. Bool M, van der Zanden R, Smit F. Factsheet preventie: Kinderen van ouders met psychische problemen (Prevention factsheet: Children with mentally ill parents), T Instituut, Editor. Utrecht: Trimbos Insituut; 2007.

14. van Veen C, van der Zanden R. Procesevaluatie Kopstoring; Een onderzoek naar de preventieve online groepscursus voor jongeren van 16-25 jaar. Utrecht: Trimbos Instituut; 2007.

15. Woolderink M, Smit F, van der Zanden R, Beecham J, Knapp M, Paulus A, et al. Design of an internetbased health economic evaluation of a preventive group-intervention for children of parents with mental illness or substance use disorders. BMC Public Health 2010;10:470 [doi: 10.1186/1471-2458-10-470]

16. Coyne IT. Sampling in qualitative research. Purposeful and theoretical sampling; merging or clear boundaries? J Adv Nurs 1997 Sep;26(3):623-630.

17. Hsieh HS, Shannon SE. Three approaches to qualitative content analysis. Qual Health Res 2005;15(9):1277-1288.]

18. Coast J, McDonald R, Baker R. Issues arising from the use of qualitative methods in health economics. $J$ Health Serv Res Policy 2004 Jul;9(3):171-176. [doi: 10.1258/1355819041403286]

19. Dickens BM, Cook RJ. Adolescents and consent to treatment. Int J Gynaecol and Obstet 2005;89:5.] 
20. Alderson P. Competent children? Minors' consent to health care treatment and research. Soc Sci Med 2007 Dec;65(11):2272-2283. [doi: 10.1016/j.socscimed.2007.08.005]

21. Woolderink $M$, van Asselt $A D$, van Schayck $C P$, van Wijmen FB. E-research: problems with anonymity and consent. Ned Tijdschr Geneeskd 2013;157(51):A6828.

22. Abma T, Nierse CJ, Widdershoven GA. Patients as partners in responsive research: methodological notions for collaborations in mixed research teams. Qual Health Res 2009;19(3):401-415.

23. Bindels J, Baur V, Cox K, Heijing ST. Older people as co-researchers: a collaborative journey. Ageing \& Society 2014;34(06):951-973.

24. Calear $\mathrm{AH}, \mathrm{Christensen} \mathrm{H}$. Review of internet-based prevention and treatment programs for anxiety and depression in children and adolescents. Med J Aust 2010;192(11 Suppl):S12-S14. 



\section{CHAPTER 4}

Clinical effectiveness of an online preventive programme for adolescents with mentally ill or addicted parents: an online randomized controlled trial

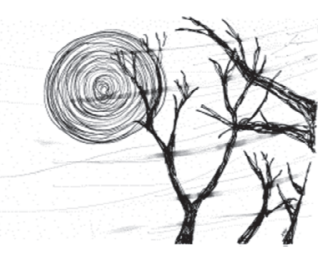

Woolderink M, van Asselt A, van Kuijk S, Evers S, van der Zanden R, Paulus A, Smit F, van Schayck CP (2016).

Clinical effectiveness of an online preventive programme for adolescents with mentally ill or addicted parents: an online randomized controlled trial.

Manuscript submitted for publication. 


\section{ABSTRACT}

Background: Preventive online interventions for youth dealing with parental mental illness are promising, as they provide an anonymous low-threshold alternative to faceto-face interventions.

Aim: The aim of this study is to evaluate the short-term clinical effectiveness of the online Kopstoring course in a randomized controlled trial.

Method: A group of 104 adolescents with parents suffering from mental illness or addiction were randomly allocated to the Kopstoring course or a wait-listed control group. The experimental condition consisted of usual care plus the online preventive Kopstoring intervention. This was compared to a control condition consisting of a waiting list with unrestricted access to usual care. Measurements were conducted at baseline and 3 months, with the Youth Self-Report (YSR) as its main outcome and the Centre for Epidemiologic Studies Depression Scale as its secondary outcome measure. Missing data were imputed.

Results: No significant between-group differences were found on the YSR internalising problems scale (depression and anxiety problems) (difference score $=-2.45 ; p=0.159$ ). However, there is some evidence that internalising problems decreased in the intervention group, whereas the control group showed few signs of improvement over the first 3 months. These differences were, however, not statistically significant. Analysis of complete cases (without imputation) showed a treatment effect on internalising problems.

Discussion: Despite the statistically non-significant between-group differences we observed a trend in the within-group change scores in favour of the intervention group. The results have been obtained under several methodological, medical-ethical and financial difficulties. Low power was a limitation in this study.

Conclusion: We found reductions in internalising symptoms scores favouring the intervention group. However, none of the outcomes reached statistical significance. Due to effects of methodological, medical-ethical and organisational problems, the results of this study have to be interpreted with utmost care. It is recommended to enrich the Kopstoring course with effective methods to address internalising problems. Future effectiveness research, including clinical outcomes as well as coping skills, are needed on this and similar interventions for this target group. 


\section{BACKGROUND}

Worldwide, mental illnesses are highly prevalent [1]. A mental illness is not only a burden for the patient but also for the family of the patient and those around them [2,3]. Parents are among those diagnosed with mental or substance abuse disorders. Furthermore, there is a body of evidence indicating that when a child is exposed to maternal or paternal disorders, he or she is at elevated risk of developing the same condition as the parent or developing another mental illness or addiction problem [4-9]. Bassini and his team estimated the number of children exposed to parental psychiatric disorders by means of a national survey [10]. The results of this survey show that in Canada, one in every ten children under the age of 12 years lives with a parent with a psychiatric disorder, and one in every six children lives in a household where at least one person is affected with a psychiatric disorder [10]. The prevalence of parents meeting criteria of mental illness or substance use disorder in the Netherlands is about 405,000 parents annually. In total, these parents have 577,000 children younger than 18 years (minors), and of these children, 423,000 are younger than 12 years [11]. These children represent $17 \%$ of the total child population $[12,13]$, that is, approximately one in every six children in the Netherlands.

Appropriate help and support for the children of these parents is important and beneficial [5,14-16]. Although preventive interventions for at-risk offspring are provided only rarely, these interventions have been developed and have proven to be effective $[17,18]$. The vast majority of these interventions, however, are face-to-face interventions, and few have been developed for online use. It is immensely difficult to recruit these youngsters for face-to-face interventions because this means they cannot participate anonymously; participation in face-to-face groups might endanger their privacy and would increase the probability that their parents learn of their attendance. In addition, participation in face-to-face interventions would include travel time. Online interventions carry the promise to overcome these obstacles. Online participation is anonymous, and users can take part from home without anyone knowing.

The Kopstoring course, provided at www.Kopstoring. $\mathrm{nl}$, is an online alternative to faceto-face interventions for children of mentally ill or addicted parents (further referred to as COPMI, children of parents with mental illness, including addiction). This course is a an adapted version of the Dutch face-to-face course for COPMI adolescents and contains elements of the online Grip Op Je Dip course for depressed adolescents [19]. Developed by four mental health institutions and the Trimbos Institute, the Netherlands Institute of Mental Health and Addiction, the Kopstoring course is qualified as 'theoretically sound' by the Dutch Committee for Youth Mental Health Interventions. The course has been designed for COPMI adolescents in the age range of 16 to 25, and results from a pilot study performed in 2007 were promising in terms of strengthening coping strategies and decreasing depression symptoms [20]. Thus, the course has been shown to be a potentially worthwhile and efficient alternative to individual face-to- 
face preventive interventions. Although the pilot study shows that the online course has potential, robust evidence for its effectiveness was needed.

The aim of this study was to assess the short-term effectiveness of the Kopstoring course over three months using data obtained from a multicentre randomized controlled trial.

\section{METHOD}

We conducted an online multicentre randomized controlled trial in the Netherlands. Details of the study's design are provided below and can be found in the design paper of this study [21].

\section{Changes to the trial design}

After start of the trial, a number of modifications in the trial design had to be implemented, causing deviations from the original design protocol [21]. The changes concerned the power calculation, the screening procedure and the choice of secondary outcome measures. All modifications were communicated with and approved by the Medical Research Ethics Committee (MREC).

Initially, screening for existing emotional and behavioural problems was performed by means of the Youth Self Report. The concept was that potential participants with existing problems would be excluded from the trial, as the Kopstoring intervention aims to prevent these problems, not cure them. However, after start of the trial the majority of potential participants scored above the cut-off-point, which led to their exclusion. Meanwhile, in clinical practice, the Kopstoring course had been successfully offered to many comparable participants without applying the YSR in the screening procedure. Therefore, applying the YSR score intended as a screening instrument, turned out to be unfortunate, as the majority of potential participants had to be excluded because they scored above the cut-off point. In addition, the a-priori choice of using the Symptom Checklist-90 (SCL-90) as a secondary outcome measure appeared to be ill-suited. The SCL-90 was too long and captured too many of the same complaints of the target population as the YSR. Therefore, the Centre for Epidemiologic Studies-Depression Scale (CES-D) was used instead, which is a much shorter instrument and focuses on capturing internalising problems. In addition, the recruitment problems and the availability of new information about the correlation of YSR measures, were reasons to reconsider the initial (conservative) power calculation. The changes are described in more detail below.

\section{Participants}

Dutch-speaking adolescents aged 16 to 25 years with at least one parent suffering from a mental disorder or substance use disorder were eligible to enrol in the online 
preventive course. Prevention is the aim of the Kopstoring course. Therefore, we excluded people meeting the diagnostic criteria of DSM-IV disorders and those who showed any indication of suicidal ideation. Professionals from 9 mental health services who offered the online intervention checked and monitored this. In addition, participants were excluded if they had no access to the internet via a computer. Written informed consent was obtained, as was additional parental consent in case of 16- and 17-year-old participants. Participants received an incentive (70 euro) for completion of all questionnaires.

As mentioned, this screening procedure is a deviation from the screening procedure described in the protocol [21] and was implemented 9 months after trial commencement. Initially, the YSR was used as a screening instrument, as per protocol, whereas after 9 months, the Effect Evaluatie Lijst (EEVL) was used as screening instrument instead of as secondary outcome measure. In addition to administering the EEVL, the potential participant was asked if they were diagnosed with a DSM IV classified disease and a check for suicidal ideation took place. In case of any doubt regarding the severity of symptoms the procedure allowed for 'extra screening questions' to determine the eligibility of the person. All other in- and exclusion criteria remained unchanged.

\section{Interventions}

In this multicentre study, we compared two groups: a group that received the intervention (named Kopstoring) and a group that was placed on a waiting list for 6 months (as explained below).

\section{Kopstoring:}

The Kopstoring program is an online course program consisting of eight weekly chat group meetings in a secure chat box. The aim of the intervention was to strengthen protective factors, such as self-management skills and psychological wellbeing, and prevent the development of psychological disorders [21]. Prevention workers and psychologists from mental health services trained in the delivery of online preventive interventions provided the Kopstoring program. The intervention was protocol driven and well structured. The course had eight themes, so each week there was a new theme. The themes were: home situation, roles in families, thoughts and feelings, questions about addiction and mental problems, different coping styles, social networks, leading your own life in relation to others, and what was coming up in the future. The participants were required to carry out homework assignments prior to every online meeting.

Waiting list (care as usual):

Participants allocated to the control condition had to wait 6 months before they could participate in the Kopstoring course. They had unrestricted access to care as usual 
while wait-listed. Participants allocated to the waiting list were monitored by the mental health service, which screened them in case symptoms increased or began manifesting.

\section{Data collection procedure}

Participants were screened for eligibility. Informed consent forms were sent out to the eligible participants, along with an invitation to fill in the baseline questionnaires. Participants were then allocated to either the intervention or the waiting list condition using a permuted block randomisation procedure $[22,23]$. At 3 and 6 months after baseline, participants were invited to fill in a set of questionnaires. Participants allocated to the intervention group had an extended follow-up with measurements at 9 and 12 months after baseline. For this paper, we analysed the effects at the 3-month follow-up. The number of respondents who filled out questionnaires at baseline, 3 months and 6 months was very low (31 respondents (29.8\%)). Due to this amount of missing data we only considered the short term effects of Kopstoring for this paper. (The high amount of missing data is further described in the discussion section).

\section{Outcomes}

Primary outcome measure:

The primary outcome measure was the Youth Self Report (YSR), which is part of the Achenbach's Child/Adolescent Behaviour Checklist from T. M. Achenbach. We used the Dutch translation [24]. This questionnaire measures internalising and externalising psychological problems in adolescents. The total score of internalising measures is the combination of three scales: anxious/depressed scale, withdrawn/depressed scale, and somatic complaints. The total score of the externalising scale is the combination of two scales: rule-breaking behaviour and aggressive behaviour. Due to a technical problem with administering the online questionnaires, one item in the externalising scale was not correctly processed. Therefore, all participants were assigned a 'missing' value on that item. We do not expect this to have caused any relevant bias, as all patients received the same score. The total score of the YSR is the sum of all nine scales. Clinical cut-off scores for the internalising scales were scores of 16 and higher for men and 21 or higher for women, and borderline clinical scores on the internalising scales were scores of 14 or higher for men and 18 or higher for women. For externalising scales, clinical cut-off scores for men were 18 or higher and for women 20 or higher [24]. The YSR was measured at baseline and at 3 months after baseline. The smallest clinically relevant difference for the YSR is 0.45 [25].

Secondary outcome measures:

The Centre for Epidemiologic Studies-Depression Scale (CES-D) was used to measure depressive symptoms [26-28]. This questionnaire contains 20 items that measure the frequency of 20 symptoms of depression on a 4-point Likert scale. The higher the score 
(range 0-60), the higher the indication of an existing depressed state. The Dutch webbased CES-D questionnaire is a reliable instrument to use in a Dutch population [29]. The CES-D was measured at baseline and at 3 months after baseline.

The initial design protocol described the use of the Symptom Checklist-90 as secondary outcome measure. The first YSR data observed during the screening phase revealed that symptoms and complaints were concentrated around the internalising problem scales of the YSR. This scale measures depression and anxiety. The SCL-90 is considered to capture these problems of the target population less accurately as opposed to the Epidemiologic Studies-Depression Scale (CES-D).

All outcomes were self-reported by the participants. We used a service-use questionnaire specifically developed for this study, which measured healthcare use and services use outside the healthcare sector.

\section{Sample size}

For the initial power calculation, as mentioned in the published protocol [21], we computed that 78 participants were required per condition, hence a total of 156 participants. This calculation was based on the ability to detect a clinical medium effect size or a larger effect size, which corresponds to a standardised mean difference (Cohen's d) of $d>0.45$ [25]. We will test the hypotheses at a conventional power of $(1-$ beta $)=$ 0.80 and alpha $=0.05,2$-tailed. Then we compensated for a loss of $28 \%$ drop-out based on the dropout rate observed in the pilot study [20] and this required a total of 214 participants at baseline.

The initial power calculation is based on the hypothesis that groups would be tested with an independent T test which appeared to be conservative. During the RCT period information became available about the correlation between the baseline and followup measurement of the YSR. Incorporating this correlation in a modified power calculation resulted in a lower sample size to be recruited.

The trial was designed to detect a clinically relevant standardised mean difference of $d=0.45$ on the YSR [25]. Setting the probability of a type-I error at 0.05 , the power at 0.80 , and assuming a correlation between pre and post-test of $r=0.7$, we computed that 40 participants were needed in each condition, thus 80 in total. We compensated for a loss of $28 \%$ drop-out based on the dropout rate observed in the pilot study [20]. This required a total of 112 participants at baseline (Appendix 2 contains the detailed sample size calculations).

\section{Data analyses}

Before analysing the data, missing data were imputed using predictive mean matching, which assures no values out of range can be provided and is robust against misspecifi- 
cation of the distribution of the data and misspecification of the imputation model [30]. We assumed data were missing at random, conditional on other covariates (see below). To test the clinical effectiveness of Kopstoring, we computed change-frombaseline scores for both groups for all major clinical outcome variables. Betweengroup effects were estimated using analysis of covariance in which we corrected for differences in potentially confounding factors between groups at baseline. As gender and age could have confounded the outcomes, we have corrected for these variables in the covariance analyses. In addition, we have corrected for the baseline differences by including the baseline clinical measures (YSR and the CES-D) as covariates. The adjusted effects were compared to crude estimates in which no correction was applied. We also determined the proportion of participants who made clinically meaningful changes on the YSR and the CES-D [31]. Cohens $d$ scores were calculated by subtracting the mean of the control group by the mean of the intervention group, divided by the pooled standard deviation from the entire sample [32]. All analyses were carried out using SPSS (IBM SPSS Statistics for Windows, Version 22).

\section{RESULTS}

\section{Participant flow}

Recruitment was conducted both online and offline, with the emphasis on online recruitment through advertisements on Facebook and Twitter as well as banners and links on websites related to the problems occurring. Offline recruitment focussed on public relation (PR) activities and consisted of articles in newspapers, local radio interviews, and so forth. PR activities of mental health services were focussed on creating awareness of the existence of Kopstoring. These activities focussed on the general population (schools etc.). Mental health providers did not recruit possible participants by using files of families dealing with parental mental illness. Recruitment began on 1 February 2011 and lasted until 31 October 2014.

Figure 1 presents the participant flow throughout the trial. In total, 299 potential participants registered for Kopstoring. After a first screening, 46 participants were excluded due to an incomplete registration procedure. An incomplete registration procedure meant that the participant failed to complete to fill in the required registration questions and 3 screening questionnaires prior to participation. In addition, one double registration was removed. From the remaining potential participants, 252 participants were randomly allocated to one of the conditions of the trial. From these 252 randomly allocated participants, 26 participants withdrew from participation and 122 participants failed to return their written consent. The remaining 104 participants, who provided both online and written informed consent were allocated to the conditions as follows: Kopstoring ( $n=55)$ or the waiting list control $(n=49)$. 


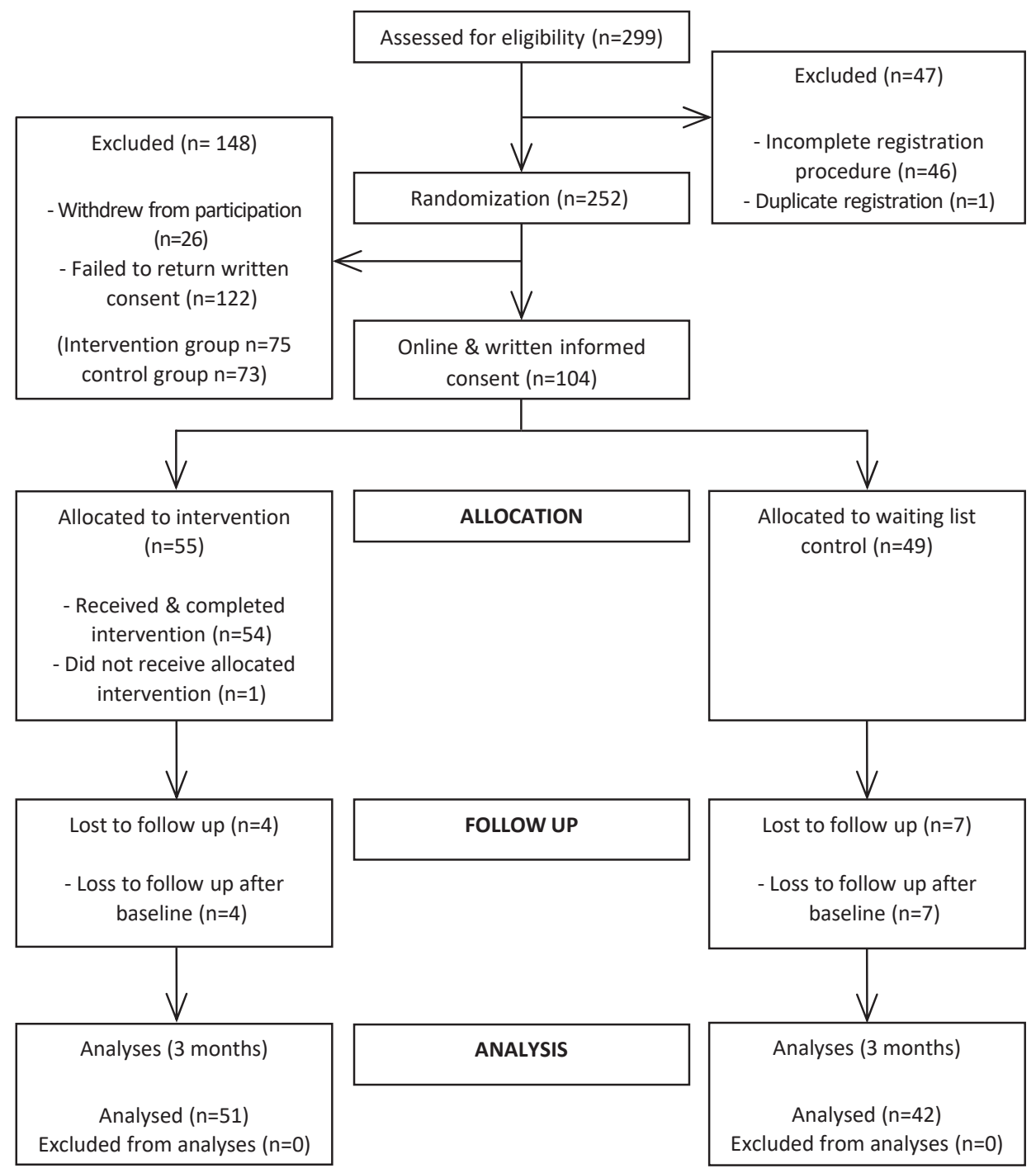

Figure 1: Participant flow chart 
As mentioned, the screening procedure has been adapted after trial commencement. Using the initial screening procedure 34 (73.9\%) out of 46 potential participants were excluded based on the exclusion criteria of scoring higher than the YSR cut-off score. No potential participant has been excluded based on the new screening procedure, however the 'extra screening questions' were used in the majority of cases to make sure participants were included based on complete information about the complaints and their situation. Participants included based on the initial screening procedure, fulfilled the inclusion criteria of the new screening procedure and were therefore eligible to participate.

\section{Baseline data}

Table 1 provides an overview of the baseline characteristics of the trial population. Despite randomisation, there was imbalance in the two groups with respect to both the nature of the problem (mental illness or addiction) and the number of parents with problems involved.

Table 1: Baseline characteristics of the total sample

\begin{tabular}{|c|c|c|c|}
\hline Variable & $\begin{array}{l}\text { Kopstoring } \\
(\mathrm{N}=55)\end{array}$ & $\begin{array}{l}\text { Control } \\
(\mathrm{N}=49)\end{array}$ & $\begin{array}{l}\text { Total } \\
(\mathrm{N}=104)\end{array}$ \\
\hline Gender Female, nr (\%) & $46(83.6 \%)$ & $47(95.9 \%)$ & $93(89.4 \%)$ \\
\hline Age (Sd) & $20.6(2.6)$ & $20.1(2.8)$ & $20.4(2.7)$ \\
\hline 16-17 year, n (\%) & $9(16.4 \%)$ & $11(22.4 \%)$ & $20(19.2 \%)$ \\
\hline 18 years and older, $\mathrm{n}(\%)$ & $46(83.6 \%)$ & $38(77.6 \%)$ & $84(80.8 \%)$ \\
\hline \multicolumn{4}{|l|}{ Nature of problem/Parent with problem } \\
\hline Mental illness, n (\%) & $29(52.7 \%)$ & $33(67.3 \%)$ & $62(59.6 \%)$ \\
\hline Maternal & $23(41.8 \%)$ & $23(46.9 \%)$ & $46(44.2 \%)$ \\
\hline Paternal & $6(10.9 \%)$ & $10(20.4 \%)$ & $16(15.4 \%)$ \\
\hline Substance abuse/dependency, n (\%) & $7(12.7 \%)$ & $2(4.1 \%)$ & $9(8.7 \%)$ \\
\hline Maternal & $2(3.6 \%)$ & $0(\%)$ & $2(1.9 \%)$ \\
\hline Paternal & $5(9.1 \%)$ & $2(4.1 \%)$ & $7(6.7 \%)$ \\
\hline Any combination of disorder \& parent ${ }^{a}$ & $19(34.5 \%)$ & $14(28.6 \%)$ & $33(31.7 \%)$ \\
\hline \multicolumn{4}{|l|}{ Nationality parent(s) } \\
\hline Both parents Dutch, n (\%) & $45(81.8 \%)$ & $38(77.6 \%)$ & $83(79.8 \%)$ \\
\hline One parent not born in NL, $\mathrm{n}(\%)$ & $5(9.1 \%)$ & $6(12.2 \%)$ & $11(10.6 \%)$ \\
\hline Both parents born outside NL, n (\%) & $5(9.1 \%)$ & $5(10.2 \%)$ & $10(9.6 \%)$ \\
\hline
\end{tabular}

${ }^{a}$ Both parents same problem, both parents different problems, both parents multiple problems, one parent multiple problems

\section{Attrition and loss to follow-up}

Attrition: Of the participants in the intervention group, one person did not begin the course directly; of the remaining 54 people, 47 attended the 8 sessions and the ninth 
(evaluation session) missing one or two sessions, and seven attended the course sessions, missing more than two sessions. We considered missing one or two sessions acceptable; therefore, the completion rate of the intervention (85.5\%) was considered high.

Loss to follow-up: From the 104 participants who were randomly allocated to one of the conditions, 11 (10.6\%) provided no data at any time (included the baseline measurement) and were therefore excluded for any further analyses. Of the remaining 93 participants, 16 (17\%) did not complete baseline questionnaires. The reason for noncompletion of questionnaires is unknown. Thirty-seven (40\%) participants had incomplete data at 3 months. There were no significant differences in completion of questionnaires between the two groups $(\chi 2=0.80 ; p=0.778 ; d f=1)$. This indicates the loss to follow-up was possibly random.

\section{Outcomes of the intervention}

Table 2 presents the clinical measures at baseline. One of the baseline imbalances we observed was that the control group scored lower on the clinical measures compared to the intervention group. This implies that the control group had an overall better state of health than the intervention group at the beginning of the study (a lower score means a better health state). To adjust for these baseline differences, we used the YSR and CES-D scores at baseline as covariates (in addition to age and gender) in further analyses.

\section{Youth Self Report (YSR)}

Table 2 shows the change generated by the intervention in terms of the mean raw scores on the YSR. The YSR scores were divided into two categories: internalising and externalising problems. The total score of the YSR is also included in the table. The emphasis of the effect of the intervention is on the difference in effect measured between groups before and after (at 3 months) receiving the intervention. 


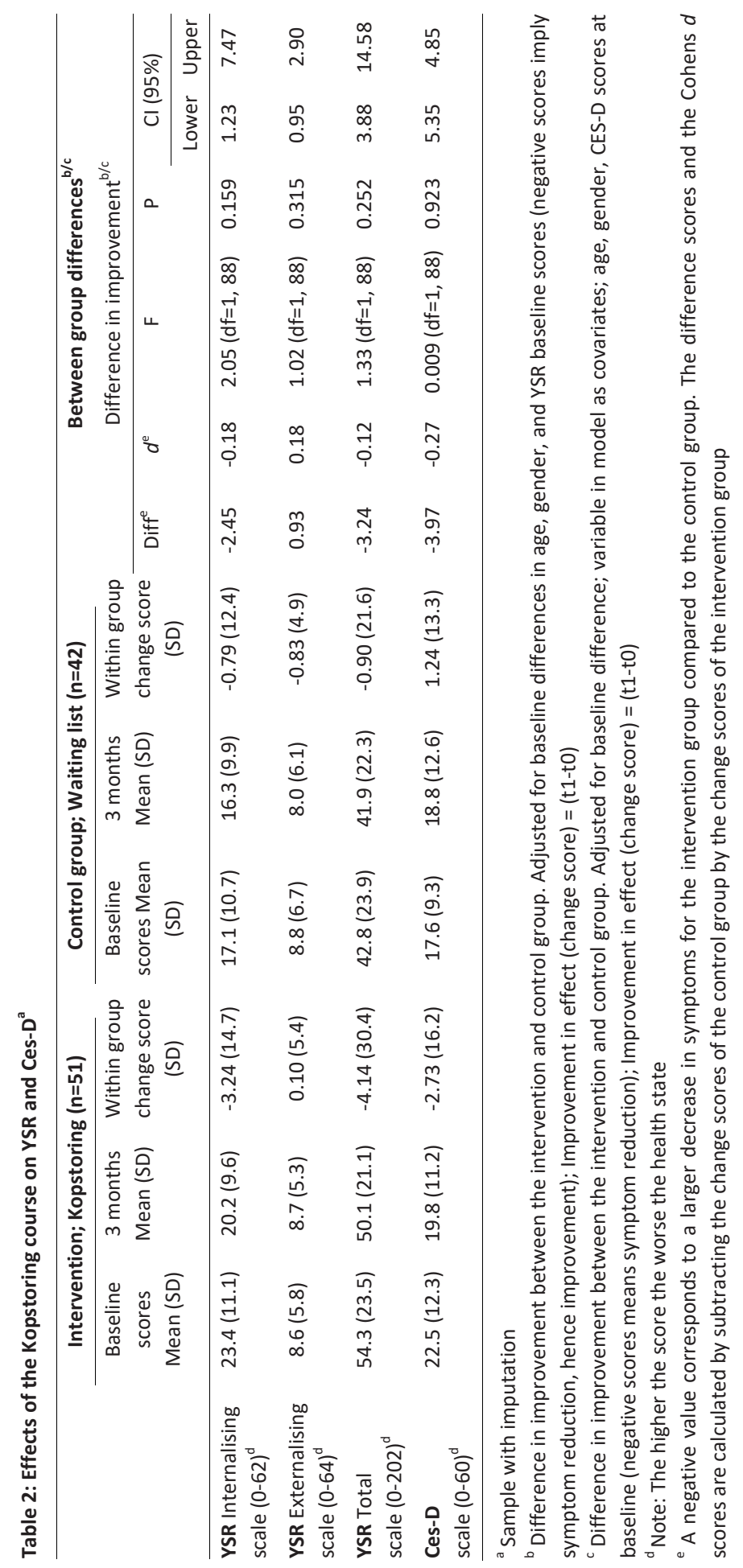


In the adjusted (covariance) analyses the outcomes show the same direction of change. The intervention group improved after receiving the intervention, as did the control group (a negative score means symptom reduction) for the internalising and total scores. For the internalising scale of the YSR as well as the total outcome on the YSR, the intervention group improved more than the control group (reflected in the negative difference and Cohens $d$ score). As mentioned, for this target group, evidence shows the focus should be on internalising problems. This is the spectrum in which also our respondents score high compared to the externalising problems dimension. Despite the absolute clinical changes observed, the improvement of the intervention group is not statistically significant compared to the changes of the control group.

\section{Centre for Epidemiologic Studies- Depression Scale (CES-D)}

In addition, table 2 shows the clinical effects of the intervention measured on the CESD. Analyses of covariance show improvement for the intervention group after uptake of the intervention while the control group shows a positive score, indicating a worse state at 3 months as compared with the scores at baseline. The between-group differences at 3 months are not statistically significant.

\section{Reliable change}

For the Youth Self Report internalising scales we calculated the reliable change scores at 3 months after baseline [31]. For the YSR internalising scales a reliable significant improvement corresponds with a negative score $(\mathrm{RC})$ and the sum score must cross the subclinical clinical threshold (male: 14; female: 18) [24]. In the intervention group, 19 of the 32 participants who showed reliable improvement, were also recovered according to the YSR cut of score. In the control group, 17 of the 21 participants who showed reliable improvement, were recovered according to the YSR cut-off score. Analyses (adjusted) show the differences between the groups were not statistically significant (OR=0.93; $p=0.881 ; 95 \% \mathrm{Cl}=0.36-2.42$ ).

For the CES-D a reliable significant improvement corresponds with a positive change score of 5 points or more and the sum score must cross the clinical threshold of 22 points to a lower score (based on Cuijpers, 2008 [33]). At 3 months follow-up, 19 (37.3\%) participants from the intervention group and 13 (31.0\%) participants of the control group showed a reliable change on the CES-D. These differences between the groups were not significant ( $O R=0.94 ; p=0.902 ; 95 \% \mathrm{Cl}=0.36-2.44)$.

\section{Complete case analyses and ancillary analyses}

In addition to the analyses we performed an (adjusted for covariates) analysis using complete cases only (i.e. without imputed score values). This showed no differences in sign of the treatment effect on the YSR internalising (diff $=-9.37 ; p=0.000 ; F=22.82 ; d f=(1.26) ; d=-$ 1.54 ) and total score of the YSR (diff=-17.64; $p=0.002 ; F=11.98 ; d f=(1.26) ; d=-1.29$ ) neither 
for the Ces-D (diff=-5.13; $p=0.232 ; F=1.499 ; d f=(1.26) ; d=-0.54)$ except for the YSR externalising scales (diff=-1.30; $p=0.430 ; F=0.644 ; d f=(1.26) ; d=-0.38)$. In the complete case analyses all differences scores (and Cohens $d$ scores) are negative indicating the intervention group shows a better improvement than the control group, however only for the YSR internalising scales and YSR total scores the effects show significant improvement in favour of the intervention group.Covariance analyses, with adjustment for baseline differences, show a significant difference between the intervention and control group at 3 months after baseline on the Youth Self report. The complete case analysis indicates that the score of the intervention group is worse at baseline than the analyses where we imputed data (23.4 (SD: 11.1) compared to complete case mean 27.7 (SD: 7.5)). The scores of the control group are slightly different (17.1 (SD: 10.7) compared to complete cases mean of 18.6 (SD: 10.1)). This analysis shows that there is an effect of the intervention after 3 months in favour of the intervention group on the internalising and total scales of the YSR

\section{DISCUSSION}

\section{Main results}

In contrast to the main hypothesis, the findings of this study show there was no significant difference between Kopstoring and care as usual during a 3-month follow-up period in terms of depressive symptoms and internalising and externalising problems. Complete case analyses (i.e., without imputed score values) replicate the same direction of effect for the clinical outcome scores but with a significant improvement in the intervention group on the internalising and total problems scale of the YSR.

\section{Comparison with other work}

This randomized trial examined the effects of an online intervention for adolescents dealing with parental mental illness or addiction. A straightforward comparison with existing effectiveness studies is hampered by the differences in target population and unit of analysis (mainly entire families instead of children). For populations in early adulthood, the effectiveness of depression prevention in the Netherlands has been demonstrated by van der Zanden et al.[34]. However, the studies assessing interventions for online depression prevention focus on the patient and not on the person's offspring or surroundings. Although some studies performed in the Netherlands focus on the COPMI, they use different study designs; therefore, a comparison of study results would be difficult [35-38]. Only one Dutch study, performed by van Santvoort et al. [39], investigated the effectiveness of a support group for COPMI, but it concerned a family intervention provided face-to-face and therefore this is not a good comparator either. 


\section{Limitations}

This study has several limitations. The randomized trial performed encountered several problems that might have influenced the outcomes of this evaluation. First, we have to conclude that this trial is underpowered. This methodological problem was partly introduced by the complicated informed consent procedure with the legal obligation to obtain written consent instead of online consent. The medical-ethical committee interpreted written consent as providing consent using a pen-and-paper medium [40]. The requirement of obtaining written consent led to several problems. Paper consent forms had to be sent out to a population that wishes to remain anonymous. These adolescents did not want their parents to know about their wish for online help for the symptoms or problems they experience because of their parents' problems. As many of the adolescents ( $51.9 \%$ of the included sample) still live with their parents, we had to send the consent forms to their home addresses. From the 226 participants who gave online informed consent, only 104 (46\%) returned their written consent for study participation. In addition, in the Netherlands, a person aged 16 and older is legally allowed to make decisions about medical treatment and is treated as an adult in the healthcare system. In contrast, for participating in scientific studies investigating the effectiveness of these medical treatments or interventions, informed consent is needed from parents until the age of 18. For adolescents aged 16 and 17, this legal inconsistency means they can decide to have treatment but need parental consent to participate in a scientific study assessing the effectiveness of that same treatment [40]. Participants aged 16 and 17 were therefore likely to be underrepresented in this trial, and it is uncertain whether results can be generalised to this age group.

A second limitation is a high amount of missing data due to incomplete questionnaires. The number of respondents who filled out questionnaires at baseline, 3 months and 6 months was very low (31 respondents $(29.8 \%)$ ). We therefore focussed on the short term effects of Kopstoring. A consequence is that we have not analysed longer term effects, which is a limitation. The reason for the high amount of missing data might be the low threshold of online evaluation studies.In contrast to what is hypothesised to be the case for online studies (online delivery is appreciated because it can be accessed whenever participants like, as many times as they like, etc. [41, 42]) we found no indication that adolescents are more responsive to or appreciative of invitations to fill in questionnaires through e-mail or online. The advantages of online data collection, for example, filling out the questionnaire in one's own time, anonymity, and speed, also seem to be its disadvantages in this case.

Thirdly, changing the screening procedure resulted in a population with moderate to severe clinical complaints, while it was expected that symptoms in the study population would be largely subclinical. This raises the question whether the Kopstoring course, with its preventive nature, is 'intense' enough to help the adolescent offspring 
of parents with mental health or addiction problems. A mismatch between course and population may have caused the absence of any effect.

In addition to this signs of a possible selection bias have been noted. The baseline scores for the internalising scales of the YSR suggest that 28 participants in the intervention group and 12 participants in the control group (total of 40 participants (43\%)) had more severe complaints than expected initially, scoring in the borderline clinical or even clinical range. This implies that the control group scored better on most of the clinical measures, especially the internalising problem scales of the YSR (which express depression, anxiety, and somatic complaints), indicating that the control group was in a better health than the intervention group at baseline. Taking this reasoning one step further could explain why the intervention group seems to have better difference scores after the intervention than the control group: naturally, if you have lower (worse) scores at baseline, the improvement is bigger. It is imaginable that in this study we have observed a natural course of events, however slightly influenced by the differences between groups at the beginning of the study.

Another limitation was the random allocation procedure, which may have been biased due to the requirement to obtain written informed consent. Random allocation took place after online informed consent, but before written informed consent. As a consequence, participants who were allocated to the waiting list condition might have been less motivated to return their written informed consent. Also, one could imagine that those in the waiting list group that experienced more problems were particularly reluctant to return the consent forms. This might have caused the imbalance in the groups at baseline.

All participants were informed before randomisation that for participants allocated to the control group the course would be available after 6 months. It could have influenced participants' expectations and feelings knowing that, in time, they too would receive the course. On the other hand, being allocated to the control condition is not an active decision of the participant, which might negatively affect mood and feelings of control.

A final limitation concerns the generalisation of results. In general, extrapolation of these trial results to another (online) preventive setting is problematic due to the level of existing clinical symptoms measured in this COPMI population. In addition, participants aged 16 and 17 were underrepresented due to the parental informed consent procedure mentioned previously. Similarly, male participants and children of parents with a foreign background were underrepresented.

\section{Implications/interpretation}

The findings of this study did not confirm the hypothesised clinical effects of Kopstoring. Although results have to be interpreted with great caution because of the limita- 
tions mentioned, the outcome of the study raises serious questions about the effectiveness of the course for clinical outcomes. A recent cross-sectional and longitudinal study by van Loon and colleagues (2015) among offspring of parents with mental illness indicated that low use of passive coping, use of active coping, self-disclosure, and parental monitoring were protective factors against developing internalising problems. Given these results and the negative outcome of the current study, it seems important to re-evaluate the methods used in the Kopstoring course to address internalising problems and depression symptoms.

Furthermore, initially the Kopstoring course was designed to strengthen coping strategies to decrease psychological problems in COPMI adolescents. Coping strategies, as an intermediary factor in the prevention of psychological problems, were not included as outcome parameter, in our study due to necessary changes to the original protocol. It might be that coping is enhanced by the course, as the pilot study on Kopstoring suggested it might be [20], and it could be that in the long term, enhanced coping results in fewer psychological problems. Therefore, future research in which coping is included is highly recommended.

Finally, the sample consisted partly of COPMI without clinical problems (scoring above the YSR cut-off points) and partly of COPMI with clinical problems (scoring below the YSR cut-off points). The mean scores of the respondents were higher than clinical cutoff points. For the adolescents without clinical problems the necessity to improve on clinical measures, as the YSR and CES-D, seems to be less obvious than for adolescents with clinical problems. Subgroup analyses could have clarified possible course effects for the clinical subgroup; however, the small sample size did not allow such analyses.

\section{CONCLUSION}

This study shows no effect of the online Kopstoring course compared to care as usual over a 3-month time span. Despite a trend in favour of the intervention group, we were unable to confirm our hypothesis that Kopstoring would lead to statistically significant results in favour of the intervention. However, this study has met a train of obstacles that translated into limitations. It is entirely possible that we have been unable to observe the true effects of the Kopstoring course due to these limitations. We therefore need to interpret the results with utmost caution.

It is recommended to complement the Kopstoring course with effective methods for dealing with internalising problems, as these symptoms are most prevalent in COPMI. For further studies examining COPMI interventions, coping skills need to be included as a outcome measure in the evaluations. A longer follow-up period is also recommended, as it could yield more information about a possible positive effect of the course, which so far remains unconfirmed statistically. 


\section{Other information}

Trial registration number: 1982 (Dutch Trial Register). This project is funded by the Netherlands Organisation for Health Research and Development, grant number 200210002. Additional funding for course provision was provided by Innovatiefonds Zorgverzekeraars grant number, 2065. The Kopstoring course was developed by the Trimbos Instituut in collaboration with mental health institutions.

\section{Acknowledgements}

The authors would like to thank he Netherlands Organisation for Health Research and Development (ZonMw) and the Innovatiefonds Zorgverzekeraars for funding the Kopstoring project. The authors would also like to thank all participants from the Kopstoring RCT. In addition, the authors would like to thank Karlijn Arntz who has done an outstanding job as National Kopstoring coordinator. We would like to thank the providers of participating mental health institutions: Eleos, Dimence, Mindfit, GGNet, Vincent van Gogh Instelling \& Riagg Zuid and Ypse Dixet for the provision of the course. 


\section{REFERENCES}

1. Bijl RV, Ravelli A, van Zessen G. Prevalence of psychiatric disorder in the general population: results of The Netherlands Mental Health Survey and Incidence Study (NEMESIS). Social psychiatry and psychiatric epidemiology. 1998;33(12):587-95.

2. Maurin JT, Boyd CB. Burden of mental illness on the family: a critical review. Archives of psychiatric nursing. 1990;4(2):99-107.

3. Tsang HW, Tam PK, Chan F, Cheung WM. Sources of burdens on families of individuals with mental illness. International journal of rehabilitation research Internationale Zeitschrift fur Rehabilitationsforschung Revue internationale de recherches de readaptation. 2003;26(2):123-30.

4. Clarke GN, Hornbrook M, Lynch F, Polen M, Gale J, Beardslee W, et al. A randomized trial of a group cognitive intervention for preventing depression in adolescent offspring of depressed parents. Archives of general psychiatry. 2001;58(12):1127-34.

5. Beardslee WR, Gladstone TRG, O'Connor EE. Transmission and Prevention of Mood Disorders Among Children of Affectively III Parents: A Review. Journal of the American Academy of Child \& Adolescent Psychiatry. 2011;50(11):1098-109.

6. Downey G, Coyne JC. Children of depressed parents: an integrative review. Psychological bulletin. 1990;108(1):50-76.

7. Rutter M, Quinton D. Parental psychiatric disorder: effects on children. Psychological medicine. 1984;14(4):853-80.

8. Weissman MM, Fendrich M, Warner V, Wickramaratne P. Incidence of psychiatric disorder in offspring at high and low risk for depression. Journal of the American Academy of Child and Adolescent Psychiatry. 1992;31(4):640-8.

9. Weissman MM, Wickramaratne P, Nomura Y, Warner V, Pilowsky D, Verdeli H. Offspring of depressed parents: 20 years later. The American journal of psychiatry. 2006;163(6):1001-8.

10. Bassani DG, Padoin CV, Philipp D, Veldhuizen S. Estimating the number of children exposed to parental psychiatric disorders through a national health survey. Child and adolescent psychiatry and mental health. 2009;3(1):6.

11. Goossens FX, van der Zanden AP. Factsheet; KOPP/KVO. Utrecht: Trimbos Insituut, 2012.

12. de Graaf R, ten Have M, Dorsselear S. De psychische gezondheid van de Nederlandse bevolking. NEMESIS-2: Opzet en eerste resultaten. 2010.

13. van Dorsselaer S, Zeijl E, van den Eeckhout S, ter Bogt T, Vollebergh W. HBSC 2005: Gezondheid en welzijn van jongeren in Nederland. Utrecht: Trimbos Insituut, 2007.

14. Olds DL, Robinson J, Pettitt L, Luckey DW, Holmberg J, Ng RK, et al. Effects of home visits by paraprofessionals and by nurses: age 4 follow-up results of a randomized trial. Pediatrics. 2004;114(6):1560-8.

15. Scott S, Spender Q, Doolan M, Jacobs B, Aspland H. Multicentre controlled trial of parenting groups for childhood antisocial behaviour in clinical practice. BMJ (Clinical research ed). 2001;323(7306):194-8.

16. Botvin GJ, Kantor LW. Preventing alcohol and tobacco use through life skills training. Alcohol Research \& Health. 2000;24(4):250-7.

17. Siegenthaler E, Munder T, Egger M. Effect of preventive interventions in mentally ill parents on the mental health of the offspring: systematic review and meta-analysis. Journal of the American Academy of Child and Adolescent Psychiatry. 2012;51(1):8-17 e8.

18. Reupert AE, Cuff R, Drost L, Foster K, van Doesum KT, van Santvoort F. Intervention programs for children whose parents have a mental illness: a review. Medical Journal of Australia. 2013;199(3 Suppl):S18-22.

19. Verdoold S, Dijke V, van der Zanden R. Opgroeien bij ouders met psychische of verslavingsproblemen. Kopp-cursus 16 t/m 25 jaar. Utrecht: Trimbos Instituut, 2007. 
20. van Veen C, van der Zanden R. Procesevaluatie Kopstoring; Een onderzoek naar de preventieve online groepscursus voor jongeren van 16-25 jaar Utrecht: Trimbos Instituut, 2007.

21. Woolderink M, Smit F, van der Zanden R, Beecham J, Knapp M, Paulus A, et al. Design of an internetbased health economic evaluation of a preventive group-intervention for children of parents with mental illness or substance use disorders. BMC public health. 2010;10:470.

22. Zelen M. The randomization and stratification of patients to clinical trials. Journal of chronic diseases. 1974;27(7-8):365-75.

23. Lipchick LG, Nicholson RA, B. Penzien D. Allocation of patients to conditions in headache clinical trials: randomization, stratification, and treatment matching. Headache. 2005;45(5):419-28.

24. Verhulst CF, van den Ende E, Koot HM. Handleiding voor de youth self-report (YSR). Rotterdam EU, editor. Rotterdam1997.

25. Lipsey MW, Wilson DB. The efficacy of psychological, educational, and behavioral treatment. Confirmation from meta-analysis. American Psychologist. 1993;48(12):1181-209.

26. Bouma J, Ranchor A, Sanderman R, Van Sonderen E. Het meten van depressie met de CES-D. Een handleiding. Groningen, the Netherlands: Noordelijk Centrum voor Gezondheidsvraagstukken, Rijksuniversiteit Groningen. 1995.

27. Knight RG, Williams S, McGee R, Olaman S. Psychometric properties of the Centre for Epidemiologic Studies Depression Scale (CES-D) in a sample of women in middle life. Behaviour research and therapy. 1997;35(4):373-80.

28. Radloff LS. The CES-D scale a self-report depression scale for research in the general population. $A p$ plied psychological measurement. 1977;1(3):385-401.

29. Cuijpers P, Boluijt P, Van Straten A. Screening of depression in adolescents through the Internet. European child \& adolescent psychiatry. 2008;17(1):32-8.

30. van Buren S. Flexible Imputation of Missing Data: Chapman \& Hall/CRC; 2012.

31. Jacobson NS, Truax P. Clinical significance: a statistical approach to defining meaningful change in psychotherapy research. Journal of Consulting and Clinical Psychology. 1991;59(1):12-9.

32. McGough, J. J., \& Faraone, S. V. (2009). Estimating the Size of Treatment Effects: Moving Beyond $P$ Values. Psychiatry (Edgmont), 6(10), 21-29

33. Cuijpers P, Smit F. Subklinische depressie: een klinische relevante conditie? Tijdschrift Psychiatry. 2008;50(8):9.

34. van der Zanden R, Kramer J, Gerrits R, Cuijpers P. Effectiveness of an online group course for depression in adolescents and young adults: a randomized trial. Journal of Medical Internet Research. 2012;14(3).

35. van Loon L, van de Ven MO, van Doesum K, Hosman C, Witteman C, editors. Factors Promoting Mental Health of Adolescents Who Have a Parent with Mental Illness: A Longitudinal Study. Child \& Youth Care Forum; 2015: Springer.

36. van Loon L, van de Ven MO, van Doesum K, Hosman CM, Witteman CL. Parentification, stress, and problem behavior of adolescents who have a parent with mental health problems. Family process. 2015, July 24; DOI 10.111 famp/12165.

37. van Loon $\mathrm{LM}$, van de Ven MO, van Doesum KT, Witteman CL, Hosman CM. The relation between parental mental illness and adolescent mental health: The role of family factors. Journal of Child and Family Studies. 2014;23(7):1201-14.

38. van Santvoort F, Hosman CM, van Doesum KT, Janssens JM. Children of mentally ill parents participating in preventive support groups: Parental diagnoses and child risk. Journal of Child and Family Studies. 2014;23(1):67-75.

39. van Santvoort F, Hosman CM, van Doesum KT, Janssens JM. Effectiveness of preventive support groups for children of mentally ill or addicted parents: a randomized controlled trial. European child \& adolescent psychiatry. 2014;23(6):473-84. 
40. Woolderink $M$, van Asselt AD, van Schayck CP, van Wijmen FC. [E-research: problems with anonymity and consent]. Nederlands Tijdschrift voor Geneeskunde. 2013;157(51):A6828.

41. Finn J, Barak A. A descriptive study of e-counsellor attitudes, ethics, and practice. Counselling and Psychotherapy Research. 2010;10(4):268-77.

42. Wat werkt bij online hulpverlening? [http://www.nji.nl/nl/Download-NJi/Wat-werkt-publicatie/Wat_ werkt_Online_hulp.pdf]. Nederlands Jeugdinstituut. 2014 [cited 23-11-2015]. 



\section{CHAPTER 5}

\section{Methodological considerations in service use as- sessment for children and youth with mental health conditions; issues for economic evaluation}

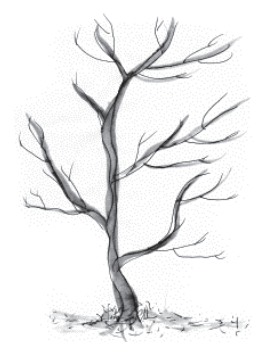

Woolderink M, Lynch FL, van Asselt AD, Beecham J, Evers SM, Paulus AT, van Schayck CP (2015) Methodological considerations in service use assessment for children and youth with mental health conditions; issues for economic evaluation.

Administration and Policy in Mental Health and Mental Health Services Research. 2015;42(3):296-308. doi: 10.1007/s10488-014-0570-4. 


\section{ABSTRACT}

Economic evaluations are increasingly used in decision-making. Accurate measurement of service use is critical to economic evaluation. This qualitative study, based on expert interviews, aims to identify best approaches to service use measurement for child mental health conditions, and to identify problems in current methods. Results suggest considerable agreement on strengths (e.g., availability of accurate instruments to measure service use) and weaknesses, (e.g., lack of unit prices for services outside the health sector) or alternative approaches to service use measurement. Experts also identified some unresolved problems, for example the lack of uniform definitions for some mental health services. 


\section{INTRODUCTION}

Mental illnesses lead to a high burden for patients and society. As more interventions, such as psychological therapies and medication become available and appear to be effective, decisions about allocation of resources within the health care sector become more difficult. Policy-makers must still operate within scarce resources, but they have a much wider range of options than 20 years ago. At the same time there is an increasing emphasis on incorporating research evidence about clinical effectiveness, efficiency and costs into decision-making. Economic evaluations are a powerful tool and can provide insights into the balance between costs and effects which can be helpful in these decisions [1]. For the measurement of cost in an economic evaluation, key steps are to identify, measure and value service use related to the illness and intervention being evaluated. Within each of these steps researchers make multiple decisions about how to operationalize these concepts. For example, in measuring the types of services used by a participant, researchers choose which services to collect data on, and which to leave out of the analysis. These decisions can have an important influence on the study results, yet there is little explicit discussion about how these decisions are made or about the pros and cons of different approaches.

Most economic evaluations of mental health interventions have focused on adult services and there are relatively few cost-effectiveness studies of interventions for children and young people in need of mental health care [2-4]. In general, the quality of these studies has been limited by narrow conceptualization of costs and perspectives $[2,4]$, perhaps reflecting the divergent opinions or approaches to service use assessment [5]. Experts recommend that economic evaluations are performed from a "societal perspective", in which all costs are included, regardless of who bears the cost [6-8]. For children with social, emotional, and behavioral problems, this societal perspective would include a broad range of services including not only health care services, but services provided in other sectors such as special education, social work, and criminal and justice systems. Adopting this "societal perspective increases the complexity of measuring service use". Investigation of the methodologies that researchers use to identify, measure, and value utilization of services can help identify problems in measuring service use and can help to guide collection of service use in future studies.

This study sought to explore the following questions about collection of service use data for use in economic evaluations of interventions for child mental health conditions. What are the best ways to collect service use data for economic evaluations? What are the strengths and weaknesses of alternative approaches? What are unresolved methodological problems in service use research that impact economic evaluations? 


\section{METHODS}

In this study, a qualitative method was employed to analyze interview data about researching the service utilization use of children in need of mental health care. Experts were defined by their expertise in the field of health services use research, and their related scientific output. Fields of expertise were deemed relevant when pertaining to (health) economics, social care and mental health care research and psychology. All experts had at least 5 years of experience in one of the related fields. Initial experts were identified through a literature search. In addition, a snowball sampling method was used. Specifically, the first interviewed experts were asked to name other experts in the field.

Experts were invited by e-mail to participate in the study. Interviews were held at the offices of the experts or over the telephone whenever a face-to-face meeting was not possible. The interviews were conducted by the lead author and were held in English or Dutch and on average lasted 1 hour. All experts gave consent for participation in the study. These qualitative expert interviews were considered exempt from review by an institutional review board.

\section{The sample}

The experts were identified in two stages. In 2009, 17 experts from the United Kingdom and the Netherlands were invited to participate in semi-structured exploratory interviews on issues related to service use research. In 2011 there was a second round of more in-depth interviews with experts from the United Kingdom, the Netherlands and the United States of America who had been identified by the 'snowball' method used in the first round. Of the 23 experts invited to participate in the 2011 interviews, six persons (14.3\%) did not respond to our invitation; one person was lost during the planning process, and one did not consider herself to be an expert related to service use of children or youth with mental health conditions.

In total, we conducted 17 semi-structured interviews in 2009 followed by 17 more structured, in-depth interviews in 2011. Five experts were interviewed in both 2009 and 2011. The total number of persons interviewed was 29 and in total there were 34 interviews (see Table 4 in Appendix 3 for an overview of sample's characteristics).

\section{The interviews}

The 2009 and 2011 interviews covered similar topics. The 2009 interviews included open-ended questions on topics related to measurement of service use research in the context of economic evaluation (see Table 5 in Appendix 4). The interviewer made written notes of participants' responses. The 2011 interviews used an interview guide which covered the same topics more systematically (see Table 6 in Appendix 5). The questions in the 2011 interviews were related to four central themes; (1) identification 
of service use, (2) measurement of service use data, (3) the valuation of services identified and measured, and (4) other problems related to service use assessment for children with mental health conditions. We recorded the participants' responses with a voice recorder and interviews were then transcribed for analysis. The transcripts were sent to the interviewees for review and the interviewee was asked to let the interviewer know if they wished to change any of their responses or if they had any additional comments they wished to provide. All respondents gave their consent for the transcript to be analyzed at the time of the interview.

\section{Data analytical procedures}

We used qualitative content analysis (QCA), which is a technique to quantify and analyze the presence, meanings and relationships of words and concepts about an area of inquiry. QCA can be used in exploratory research to describe concepts as perceived by respondents and is a common technique for analyzing semi-structured interviews [9]. QCA has been used in numerous research fields [10] including nursing sciences and allied health research [11]. Qualitative methods are increasingly used in health services research [12-15]. We chose QCA because this method helps to provide an in-depth understanding about underlying perspectives which cannot be easily obtained through most quantitative tools. Qualitative methods are inductive and reflexive and allow use of quotes [16]. These features enabled us to gain a deeper understanding of the methodological approaches and problems associated with identifying, measuring, and valuing mental health service use by youth for use in economic evaluation. We were primarily interested in describing researchers' experiences and perspectives about collection of service use data. Based on this goal, we chose to use a QCA approach similar to that described by Zhang and Wildemuth 2009. However, we also wanted to capture any new information that might have been discussed in addition to defined topics. Thus we also followed the procedure suggested by other experts in QCA [17] and concluded our analysis by looking for new more interpretive themes.

We applied the approach to QCA as described by Zhang and Wildemuth (2009). After collecting the interview data, we prepared the transcripts and materials from the interviews for analysis. The unit of analysis was the individual researcher. We developed our coding categories using previously developed categories (e.g. use of the societal perspective), and later we defined new codes based on themes emerging from the data. We tested the coding scheme on several transcripts, and then coded all text. We explored the validity of our findings using triangulation between multiple coders. Specifically, each interview was coded by 2 interviewers and we achieved researcher triangulation by discussing the key concepts, the coding scheme, and other related outcomes (MW, FL, TVA). We reviewed the analyses and conclusions to obtain final agreement. In addition, we conducted a "member check" where we asked several respondents to review the results to see whether or not the results were consistent with the participant's experience and understanding. 


\section{RESULTS}

In order to answer the questions "What are the best ways to collect service use data for economic evaluations?" and "What are the strengths and weaknesses of alternative approaches to collecting service use data?" we examined participants' responses to questions related to three subjects: identification of service use, measurement of service use and valuation of service use. The results relating to these categories are reported in "Identification of Service Use, Measurement of Service Use, and Valuation of Service Use" section below. However, overarching themes also emerged and we discuss these in "Absence of Standardized Method, Lack of Time and Resources, No Clear Typology Exists for Services, Research Perspectives are Limited, Reflecting a Gap Between Theory and Practice and Scope of Assessment" section.

\section{Identification of service use}

First we asked experts to define "identification of service use". Most experts had similar definitions, for example one participant said the following: "identification of service use" is determining "what services and support services your particular patient population use". Respondents suggested that this meant deciding on which services to include or exclude from a study. The methods fell into three approaches: (1) studying the literature to explore current practice; (2) using formal or informal surveys or interviews to ask the target population or their proxies; and (3) using focus groups or surveys to gather information from professionals, such as clinicians, who work with the target population. Respondents said they used methods interchangeably because there is no guideline in this area and often used a method that was either familiar or simple to implement. Some participants reported using only one method while others reported using all three methods. Respondents indicated that their choice of how to identify the services likely to be used was often driven by time and money constraints. They agreed that there was a lack of a gold standard about how to identify the range of services that might be used. A lack of gold standard means that there might not be a consistent way in what is included in a services assessment and this in its turn makes reports of different studies harder to compare.

A subset of respondents questioned who the unit of observation should be (the person from whom data is gathered) and who the unit of analysis should be (persons to whom findings are generalized) when studying young populations. Most agreed that the individual child/youth should be the unit of observation and analysis but some also thought that service use and costs for others, such as parents, should be included in the assessment. Differences in the unit of observation or analysis adopted may lead to (or be the result of) variation in study design and the costs measured will be different depending on whether or not the unit of observation is limited to the child or includes 
other family members; if this is not explicitly stated it can cause difficulties when comparing findings across studies.

\section{Measurement of service use}

Participants were asked to define "measurement of service use" and describe available service use data collection methods and then identify problems related to these methods. Participants agreed on the definition of "measurement of service use". One participant defined it as follows:

"Measurement is the process by which you go about assessing the duration and frequency of resource use and support. So for all elements you have actually identified as possible impacts on the patient group you need now go about actually defining what that means in terms of the intensity and frequency of access."

When asked about problems measuring service use, participants agreed there was no clearly preferred approach or instrument to measure service use.

Most participants mentioned the importance of measuring service use broadly, which entailed including all costs relevant to "services across the whole range of sectors". But they also raised issues related to broad measurement of service use, including time constraints and lack of good assessment tools for service use in some sectors, such as social welfare services.

Participants discussed two forms of data collection; direct measurement through interview instruments or selfreport questionnaires, completed by either the youth or their proxies, and indirect measurement through secondary data sources such as hospital records or electronic medical records. Figure 1 presents this information. 


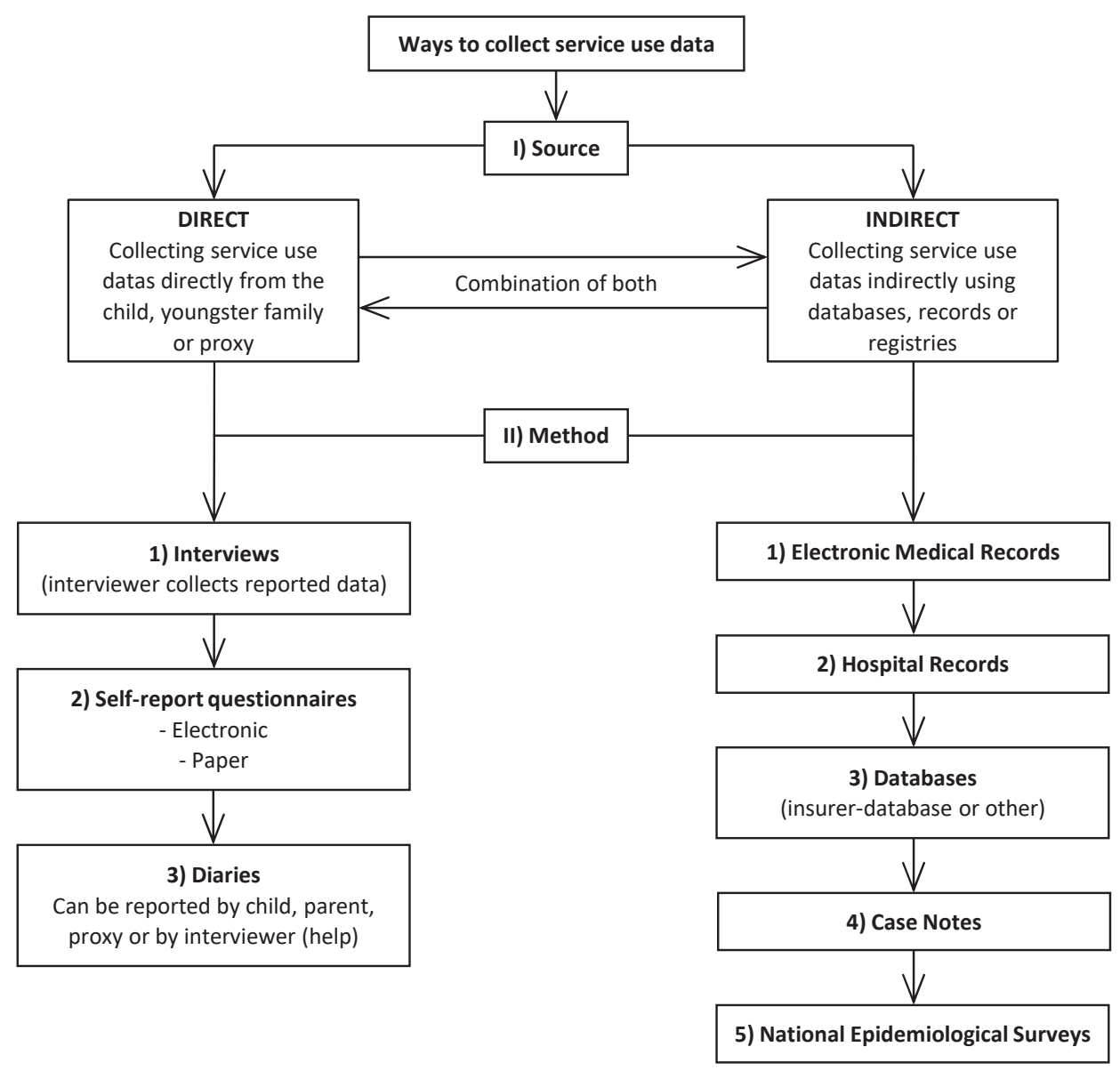

Figure 1: Direct and indirect methods of service use data collection

Table 1 lists the strengths and weaknesses of direct and indirect methods. Direct data collection was identified as the most comprehensive and strongest approach by most respondents. However, experts identified several important challenges to this approach. For instance, experts expressed concern about participant recall, particularly if there was a long time between data collection points. In addition, the decision about whether or not to use proxy respondents was raised. For instance, in younger children having the parent report about which services the child used is likely more accurate than having the child report services. Another concern most experts noted was that there was no one measurement instrument that was preferred and that instruments varied considerably, with differences in comprehensiveness, degree of testing prior to use, and method of delivery (e.g. interviews, mail surveys, or Internet surveys). Experts also noted that funding is often limited so direct measurement, such as comprehensive interviews with participants or their parents may not be feasible. For example, one 
respondent discussed the process for selecting a method for collecting service use and noted the importance of funding being a key factor in the decision:

"But those kinds of decisions typically are made in light of the other concerns and resources of the study..."

Experts also identified several advantages of indirect methods of obtaining service use data (e.g. hospital records), including reducing participant burden. However, respondents reported challenges to this method as well. In particular, obtaining records from a broad array of agencies or organizations was described as difficult or even impossible, given the time and financial constraints in most research studies.

"There is not just one file that relates to a single child where there would be records of every time they saw a social worker or their GP or a psychiatrist... All these records are kept in all sorts of different places, another responsibility of different agencies as well, so actually the reality is actually having to go in and trying to access them might be so labourintensive that it is not worth doing..."

Participants said that obtaining permission to access databases from agencies was often difficult. Discussing negotiations with agencies to get access to databases, one participant described the following issues:

"Trying to get permission from multiple sources of service use.... (It would be health in each area, and probably several different health and mental health and primary care and then also education, individual schools, social services...). It's only doable if you are doing... a project that involves services in one area. But even then it can take months to get through... And they quite often insist every single patient has to give consent. And even when you have ethical permission not to do that." 
Table 1: Strengths and weaknesses of direct and indirect data collection sources

Strengths Weaknesses

Direct sources

Able to collect data on many types of service use at once

Able to collect data on issues related to service use such as indirect costs (e.g., travel costs), productivity losses, quality of life etc.

Direct sources are considered to be the strongest level of evidence

When broad perspective is used and societal costs are measured direct sources are most practical to use
Recall problems (time, frequencies and type of service)

Possible Issue with the completeness, reliability and validity of specific instruments

Whom do we ask? Parent? Child?

Issues of reconciling parents and child reports if they differ What is the minimum age for a child to report own data?

Clinical investigators have already defined how a service is defined and measured when economist completes the team. At this point hard to introduce new measures

Indirect sources

Already collected, therefore potential cheaper and less time consuming

If registers are filled out well, they can be reliable

No issue with respondents trying to report in a socially desirable way

No issue with respondent recall problems

Not time consuming for the patient

Useful for deriving long term effects of a condition which are unlikely to be observed in a research study
Impractical to use when a broad perspective is used (impossible to get administrative data from multiple agencies for all relevant services)

Ethical considerations (e.g. informed consent and privacy issues) make it difficult to access records

Issue with the completeness, reliability and validity of data systems

Very unlikely to have records on indirect costs (e.g. travel costs, waiting time etc)

Systematically missing data in administrative records

Makes it hard to be part of the decision-making process at the start of the study about what is collected

Interpretation issues

If participants heave different health insurers, several databases may be needed, and these may not hold comparable data

Databases in general not open for researchers (used for organizations own internal affairs) 


\section{Valuation of service use}

The third set of questions concerned the valuation of services. There was no one uniform or common definition given by respondents for what "valuation of services" means. This may have been due to the open-ended way in which the question was asked. Clinician respondents seemed to focus on valuing the clinical quality of the service, and economists and health services research respondents focused more on the monetary valuation of a service. We clarified that the focus was on economic or monetary valuation of services. Not all respondents felt comfortable discussing the monetary valuation of services, as they did not consider themselves experts in this aspect of service use research. One economist provided this definition:

"Valuation is about applying costs to each unit of service use that has been identified and measured."

Respondents who discussed monetary valuation mentioned three methods to obtain a unit price for services: (1) standardized unit cost book (e.g. Handleiding voor kostenonderzoek $[18,19]$ (2) literature-based unit costs; and (3) micro costing to derive unit costs. Most of the respondents who answered the valuationquestions werefamiliarwiththese methodsandhad used all three of them at some time in their career. Table 2 reports the main problems that respondents identified with respect to alternative valuation approaches. Respondents reported that in general they were able to use each valuation method without major problems; however, they stressed that having a standardized unit cost list/book greatly increases transparency in the cost estimation process and helps ensure consistency of cost figures within a country or state. In the absence of a standardized unit cost list or book, researchers often create their own costing handbook, which makes it more difficult to compare cost estimates from different studies even if they are related to the same mental illness. Respondents also noted that valuation for some services (e.g. medical care) is easier because there are reliable sources for unit costs. In comparison, there are few or no good sources for unit costs for other services, such as juvenile justice. 
Table 2: Common problems with valuation of service utilization of children and youth

Valuation of services

Uniformity (or lack) of valua-

tion/national applicable unit cost

Lack of transparency and nontrans-

ferability of unit prices
Is this an issue in researching youth service utilization?

\author{
Yes $(92 \%, 11 \text { out of } 12)^{a}$
}

"For medical costs there are certain costs and figures attached to certain unit of services, but for human services, school services and criminal justice there are no figures attached to it so we had to develop those ourselves"

$$
\text { Yes }(66 \%, 8 \text { out of } 12)^{a}
$$

"And then there are issues in terms of... if it is you are doing multisite studies and you are looking at values across countries..."

${ }^{a}$ Not all respondents spoke about valuation (not all respondents felt comfortable discussing the monetary valuation of
services, as they did not consider themselves experts in this aspect of service use research

\section{Overarching Themes}

The overarching themes in service utilization research identified by the experts include lack of a consistent method to identify and measure service use, lack of time and resources, lack of typology for services, limited perspectives, and an undetermined scope of service to include in assessments (Table 3). Some of these issues are problematic in both adult and youth research populations, but we discuss them only in the context of youth populations.

Table 3 Themes which are important in all aspects of service utilization research in children and youth (not limited to one step of the process, therefore called overarching)

Theme

Brought by \# Words used to identify (Transcripts are fully read and

and \% experts these words are searched in order to make sure no codes were left out)

General

No methodological gold standard to

identify and measure service utilization

Lack of time and resources hamper

study methodology

No clear typology for services (not

for patient nor professional)

Theory versus practice

Limited perspectives of research

$16(94 \%)$

Undetermined scope of service use assessment

$16(94 \%)$

$10(58 \%)$

$7(41 \%)$

perspective, practice, theory

$16(94 \%) \quad$ Extent, broad measurement and identification, inclusion services in assessment, sectors, service use 


\section{Absence of standardized method}

Respondents agreed that there was no gold standard for determining what should be included in an assessment of service use in children and youth in need of mental healthcare. Moreover, the experts were unable to identify any one particular instrument or approach that could clearly identify and measure service use, most had designed or adapted their own. "There is no uniform and standardized source for these assessments," one respondent said.

\section{Lack of time and resources}

Many experts noted that research resources (e.g. time, funding) are limited and as a result, cost assessments are often designed to fit within these constraints, rather than using the best approach from a scientific point of view. Several participants noted that it was common for a cost assessment to be an add-on to an already designed clinical study. Several participants noted that many decisions regarding the design and the conduct of an economic evaluation were often influenced by these practical constraints.

\section{No clear typology exists for services}

A few experts noted that there were no clear, consistent definitions for many common services. As a result, services are inconsistently labeled across studies, making comparison of patterns of service use difficult.

"One of the big issues is that we don't have a good typology for what constitute(s) a service. They are defined differently across our different services sectors. So within the specialty mental health we will have a service which is called intensive case management that will be defined in terms of who will be providing it, how often, what they do with the families...But that could have the same name and yet be offered in the child welfare system or juvenile justice system to basically the same kind of kids and yet be defined very differently."

\section{Research perspectives are limited, reflecting a gap between theory and Practice}

All respondents commented on the various perspectives that could be used in service use research related to children with mental health conditions. All experts endorsed the societal perspective in economic evaluations. The societal perspective includes all costs of services related to the patient, family, or society $[7,8]$ regardless who pays for or receives them [20]. Experts preferred the societal perspective because it provides the most comprehensive view of costs related to interventions [1]. In the words of one expert: 
"I think you should use a societal perspective... From an economics and academic point of view that should be the perspective we would take."

However, many respondents explained that, although the societal perspective is preferred in theory, in practice it is often not used in their studies. Other factors - including the goals of the funding organization, the objectives of the clinical study, and time and resources available - determine the perspective and scope of a study.

"In many instances... there is a policymaker that is quite interested in this study and wants to know how it affects their particular agency or that kind of thing."

Even within the same setting, different perspectives lead to different estimates of service use and costs.

\section{Scope of assessment}

Linked to the issue of perspective, but focusing on services, the experts also discussed how broadly researchers should look when measuring service use among youth with mental health conditions. They agreed that services outside the regular health care sector, such as juvenile justice and school services, should be assessed, citing literature that links mental illness with the need for juvenile justice, police, and education services $[21,22]$. Most respondents said that, in theory, they preferred to collect data on a broad range of services in the assessment.

"I'm interested in all mental health problems whether or not they reach the criteria for psychiatric disorder. And all services, not only mental health services."

However, in practice, this is not always done:

"Fundamentally, it depends on the design of the clinical study, on whether they have the resources to do anything very sophisticated."

\section{DISCUSSION}

Health care decision-makers face growing pressure to incorporate research-based evidence about clinical outcomes and costs when choosing which interventions to provide to children and youth with mental health conditions [2]. One critical component of researchbased evidence is accurate data on the use of various services used by these children. Our study revealed that researchers use multiple approaches and methods to measure service use, yet there are few guidelines to help researchers de- 
cide which methods may be most accurate for a given decision or condition. Future research on some of the methodological issues raised by the experts could improve assessment of service use.

There is a clear need of research to help clarify and define services more consistently. Some services, such as medical services, are relatively clearly defined, however, other services have much less consistent definitions (particularly social or school-based services). Research to improve understanding differences in definitions could help future studies collecting service use data. For instance, when clinicians, other service providers, and researchers talk about a services, such as "case management", are they talking about the same service? Or are they using different definitions? If services are not defined and categorized consistently, costs are likely to be inconsistent as well, since different unit costs would be attributed to different services or components. These differences could lead to different conclusions for economic analyses and the decisionmaking process. To some extent problems in definitions of services could be mitigated by explicit discussion about each type of service by clinicians and researchers at the time of the study. However, in some cases, particularly when secondary data is used to examine service use, this might not be possible. Research to improve understanding of differences in definitions could help future studies collecting service use data.

Another area where future research could be useful is research to improve the instruments and methods used to collect service use. Experts indicated that projects often developed a service use data collection tool for the individual study, and these often lack information on reliability and validity of the approaches or instruments to measure service use. Greater emphasis on use of validated instruments, when applicable, would improve comparability of studies. There is now a website where many resource use questionnaires are gathered together (http://www.dirum.org/), but only a few have published data on reliability and validity [23-25]. Further, many instruments are validated or standardized for the specific populations for which they were developed, and too often they are used for other clinical groups and circumstances without prior testing [26]. Additional research on the reliability and validity of common service use measures could improve both the quality and comparability of studies collecting service use for children with mental health conditions.

The experts also identified a number of issues that could be addressed in future research to help improve the quality of service use data collected in interviews. Research on patient recall and the timing of data collection for different types of services, to what degree the structure of the questionnaire influences response, whether the setting for the interview is important, and how much training is necessary for accurate administration of interviews would be very useful. Work is also needed to identify the best method for combining and interpreting service use data that comes from multiple sources, for instance combining data from parents and children, or direct and indirect sources. 
Finally, we note a specific issue related to unit costs. Although respondents did not raise many methodological issues associated with the monetary valuation of services they indicated a clear need for development of uniform definitions of what exactly should be included in the calculation of a unit cost. If the building blocks of these cost calculations were standardized and uniform across studies, results would be more comparable and cost figures might be transferrable to other contexts. For example, there is no agreement nationally or internationally about how indirect costs such as waiting time, travel time etc., should be estimated. Standardized unit cost lists and books provide much greater comparability across studies, but these are available for only a subset of services in some countries, and commonly focus on public sector costs only. Future research should also consider the disadvantages of creating standardized unit costs. For example, some experts have suggested that using standardized costs might result in a loss of information about variability in cost between different services/providers/locations $[26,27,5]$. Thus in studies seeking to evaluate expanding services to a setting that is very different than the one in which it was originally developed, local unit costs may be appropriate. On the other hand, when comparing different services to one another within an agency, having standardized unit costs may be more appropriate.

\section{Limitations}

We interviewed only American, British, and Dutch experts, which may have influenced the results of the study. However, these experts have contributed a significant number of articles to the evidence based on mental health care service use and costs for children and youth with mental health conditions. A second limitation of the study was that both face-to-face and telephone interviews were used. It is possible that, for example, the face-to-face interviews may have led to a deeper discussion of some issues compared to the telephone interviews. To overcome this, clarification of some issues were requested by email from telephone informants and all participants were offered the opportunity to read the transcript of their interview and provide additional comments.

\section{CONCLUSION}

The aim of this study was to investigate the problems in service use research focusing on children and youth with mental health conditions. This study represents a first step in a larger discussion about how to improve methods for service use research. Our findings suggest that there are a number of unresolved issues in collecting service use data. Addressing these will help improve the quality and consistency of the data that underpin economic evaluations of services for children and youth in need of mental health care and thus also improve the quality of information that is available to decision-makers. 


\section{Acknowledgements}

The authors would like to thank Ceilidh Nichols (USA) and Inge Derhaag (NL) for their help transcribing the interviews. This project is indirectly funded by the Netherlands Organisation for Health Research and Development (ZonMw). Grant Number 200210002. 


\section{REFERENCES}

1. Drummond, M., McGuire, A. (2001). Economic evaluation in health care; Mergig theory with practice. Oxford. Oxford Universit Press

2. Beecham, J. (2014). Annual research review: Child and adolescent mental health interventions: A review of progress in economic studies across different disorders. Journal of Child Psychology and Psychiatry, 55(6), 714-732. doi:10.1111/jcpp.12216.

3. Knapp, M. (1997). Economic evaluations and interventions for children and adolescents with mental health problems. Journal of Child Psychology and Psychiatry, 38(1), 3-25.

4. Romeo, R., Byford, S., \& Knapp, M. (2005). Economic evaluations of child and adolescent mental health interventions: A systematic review. The Journal of Child Psychology and Psychiatry, 46(9), 919-930.

5. Beecham, J., \& Knapp, M. (2001). Costing psychiatric interventions. In G. Thornicroft, C. Brewin, \& J. Wing (Eds.), Measuring mental health needs. Oxford: Oxford University Press.

6. Anderson, R., \& Newman, J. F. (1973). Societal and individual determinants of medical care utilization in the United States. Milbank Memorial Fund Quarterly, 51(1), 95-124.

7. Drummond, M., Sculper, M. J., Torrance, G. W., O’ Brein, B. J., \& Stoddart, G. L. (2005). Methods for the economic evaluation of health care programmes (3rd ed.) Oxford: Oxford Universit Press.

8. Gold, M., Siegel, J., Russel, R., \& Weinstein, M. (1996). Costeffectiveness in health and medicine. New York: Oxford University Press.

9. Krippendorf, C. (Ed.). (1980). Content analysis: An introduction to its methodology. Beverly Hills: Sage.

10. Zhang, Y., \& Wildemuth, B. (2009). Applications of social research methods to questions in information and library science. Library and Information Science Research, 35, 159-170.

11. Hsieh, H. F., \& Shannon, S. E. (2005). Three approaches to qualitative content analysis. Qualitative Health Research, 15(9), 1277-1288. doi:10.1177/1049732305276687.

12. Weiner, J., Amick, H., Lund, J., Daniel Lee, S., \& Hoff, T. (2010). Review: Use of qualitative methods in published health services and management research: A 10-year review. Medical Care Research and Review, 68(3), 3-33.

13. Obermann, K., Scheppe, J., \& Glazinski, B. (2013). More than figures? Qualitative research in health economics. Health Economics, 3, 253-257.

14. Eddama, O., \& Coast, J. (2009). Use of economic evaluation in local health care decision-making in England: A qualitative investigation. Health Policy, 89(3), 261-270.

15. Bryan, S., Williams, I., \& Mclver, S. (2007). Seeing the NICE side of cost-effectiveness analysis: A qualitative investigation of the use of CEA in NICE technology appraisals. Health Economics, 16(2), 179-193.

16. Coast, J., McDonald, R., \& Baker, R. (2004). Issues arising from the use of qualitative methods in health economics. The Journal of Health Services Research and Policy, 9(3), 171-177.

17. Graneheim, U., \& Lundman, B. (2004). Qualitative content analysis in nursing research: Concepts, procedures and measures to achieve trustworthiness. Nurse Education Today, 24(2), 105-112.

18. Oostenbrink, J. B., Bouwmans, C. A. M., Koopmanschap, M. A., \& Rutten, F. F. H. (2004). Handleiding voor kostenonderzoekmethoden en standaard kostprijzen voor economische evaluaties in de gezondheidszorg. Diemen: College voor zorgverzekeringen.

19. Hakkaart-van Roijen, L., Tan, S. S., \& Bouwmans, C. A. M. (2010). Handleiding voor kostenonderzoek Methoden en standaard kostprijzen voor economische evaluaties in de gezondheidszorg.

20. Fox-Rushby, J., \& Cairns, J. (2005). Economic evaluation. New York: Open University Press.

21. Burns, B., Costello, E., Angold, A., Tweed, D., Stangl, D., Farmer, E., et al. (1995). Children's mental health services use across service sectors. Health Affairs, 14(3), 12.

22. Hoagwood, K. (1994). Introduction to the special section: Issues in designing and implementing studies in non-mental health care sectors. Journal of Clinical Child Psychology, 23(2), 114-120. 
23. Farmer, E., Angold, A., Burns, B., \& Costello, E. (1994). Reliability of self reported service use: Testretest consistency of children's responses to the child and adolescent service assessment (CASA). Journal of Child and Family Studies, 3(3), 18.

24. Jensen, P. S., Hoagwood, K. E., Roper, M., Arnold, L. E., Odbert, C., Crowe, M., et al. (2004). The services for children and adolescents-parent interview: Development and performance characteristics. Journal of the American Academy of Child and Adolescent Psychiatry, 43(11), 10.

25. Hoagwood, K., Jensen, P., Arnold, E., Roper, M., Severe, J., Odbert, C., et al. (2004). Reliability of the services for children and adolescents-parent interview. Journal of the American Academy of Child and Adolescent Psychiatry, 43(11), 1345-1354.

26. Byford, S., Leese, M., Seivewright, H., Cameron, S., Jones, V., Davidson, K., et al. (2007). Health economics letters comparison of alternative methods of collection of service use data for the economic evaluation of health care interventions. Health Economics, 16, 6.

27. Patel, A., Rendu, A., Moran, P., Leese, M., Mann, A., \& Knapp, M. (2005). A comparison of two methods of collecting economic data in primary care. Family Practice, 22(3), 323-327. 



\section{CHAPTER 6}

\section{E-research: problems relating to anonymity and consent}

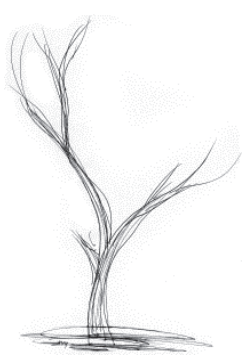

Woolderink M, van Asselt AD, van Schayck CP, van Wijmen FC (2014)

E-research: problemen rond anonimiteit en toestemming.

Nederlands Tijdschrift voor Geneeskunde. 2013;157(51): A6828. 
The Dutch Medical Research Involving Human Subjects Act (WMO) requires the written consent of participants involved in medical research. This makes online research - e-research - unnecessarily complicated and, at times, impossible. A great deal of e-research involves online interventions focusing on sensitive issues, such as sexual dysfunction, addiction and child abuse. The assurance of anonymity is an important precondition for participation in this type of research. However, the word 'written' in WMO protocols is interpreted strictly as 'on paper', and participants are not permitted to give consent through other means (e.g. email). A second requirement, which complicates research among minors aged 12 to 18 in particular, is that both parents or the guardian must also give written consent. This undermines the principle of anonymity to such an extent that finding participants is virtually impossible. While participants in medical research must be identifiable to the researcher, their anonymity must otherwise be guaranteed as far as possible. The law must be amended to keep pace with the demands of our time.

Online (e-health) interventions are on the increase [1]. These involve not only cognitive behavioural therapy, quit-smoking courses and strategies for coping with depression, but also interventions for sensitive issues such as sexual dysfunction, child abuse, or dealing with addicted or mentally ill parents. As with all health interventions, research on the effectiveness and costs of e-health interventions is important. This research itself often takes the form of e-research, enabling the researcher to track care practices online without the need for physical intervention.

Like other medical research involving human participants in the Netherlands, eresearch is subject to the WMO. This law requires that research involving human participants - including all experiments and a great deal of observational research is of sound design, that the experimental subjects are not exposed to unacceptable risks, and that they give written consent on the basis of adequate information. Proposals for e-research projects are reviewed by 1 of the 25 recognised medical research ethics committees (MRECS) in the Netherlands. If a proposal fails this review, the researcher can appeal to the Central Committee on Research Involving Human Subjects (CCMO).

An example of online research into an online intervention was the evaluation of the cost-effectiveness of Kopstoring, a course for children of mentally ill or addicted parents [2]. Some of the participants in this study live at home with one or both parents, and purposefully sought help online due to the anonymity offered by the internet. This anonymity would be jeopardised if the participants were required to sign a written consent form, particularly in the case of minors, whose parents or guardian would also have to sign. This high threshold for participation, in turn, would render the research impossible. The researchers proposed to inform the participants in advance about both the Kopstoring intervention and the cost-effectiveness research, after which they could give informed consent online. However, both the MREC and the CCMO rejected 
this proposal. Despite acknowledging the importance and usefulness of the research, both committees required that the participant and, in the case of minors, both parents were to give written consent (i.e. on paper).

This example illustrates the problems that arise from the implementation of the WMO for e-health interventions and e-research.

\section{Written consent}

The procedure for preventive review of medical experiments developed in the 1960s in the United States, and was introduced in the Netherlands in the 1970s. At the time, it was a matter of self-regulation, involving voluntary participation on the basis of sound information, as laid down in the Declaration of Helsinki of 1964. The internet did not yet exist.

In the Netherlands, the WMO came into effect on 1 December 1999 after a number of modifications. The purpose of this law is to protect the human subjects of medical research and to ensure the soundness of the research in which they are involved [3]. There has been debate about the scope of the law, especially about the definition of scientific research as research 'in which persons are subjected to treatment or are required to behave in a certain manner [4].' This type of research requires the written consent of the participants; Article 6 of the WMO expressly prohibits medical research involving subjects who have not given written consent. This is unequivocally echoed in the CCMO manual, which adds to the WMO definition the 'Guidelines for Good Clinical Practice' specifying that written consent means a signature from the subject or his/her legal representative.

Given the advent of the internet and its ensuing transactions and interventions, including e-health, this strict interpretation of the term 'written' in the WMO has become problematic. Article 6:227a of the Dutch Civil Code (BW) recognises email as written communication [5]. Moreover, digital consent is now accepted as a legal form of written consent in other areas, such as online shopping. The question therefore arises as to whether written consent in the form of an online signature, a click of a button or a scan is also acceptable in medical research.

This was the key question at a hearing of the CCMO ensuing from the case of the Kopstoring project. The CCMO followed the reasoning of the relevant MREC, adhering to the definition of written consent as '... a signature of consent on a paper medium. A broader interpretation is not permissible [6,7].' Nevertheless, the CCMO's conclusion was ambivalent: 'On the one hand, it is important that all MRECs interpret the term written in the same way. Different views should not be permitted to lead to different outcomes on such a fundamental aspect of the protection of experimental subjects. On the other hand, societal developments should be taken into account. The committee shall therefore assess the degree to which electronic consent should be possible in 
particular circumstances, and what conditions should be attached to this [7].' This suggests that the CCMO recognises the importance of broadening the conception of written consent, but considers this a task for lawmakers.

\section{Identifiability}

The requirement that consent must be given in writing relates to the identifiability of the participant. The CCMO and the MREC argue that a signature involving pen on paper is necessary to ensure that the participant is a real person. In contrast, other laws requiring consent, such as the Medical Treatment Contracts Act (WGBO) and the Data Protection Act (WBP), leave open the form of this consent.

The brochure Medical Research: General Information for Research Participants, published by the Dutch Ministry of Health, Welfare and Sport, states the following: 'Any personal data gathered by the researcher during the course of the research remains confidential. The researcher stores your data under a code. This code is used in any reports on the research. Only the researcher knows the code given to your data [8].' This means the participant's data are processed and presented anonymously, but participation in the research as such is not anonymous. That would be impossible, if only because the researcher needs to able to ascertain that the participant meets the inclusion criteria. In addition, the researcher must be able to verify data with the participant if needed. Medical researchers are required to adhere to the Dutch Code of Conduct for Health Research in doing so $[9,10]$.

In short, participants in medical research, including online research, must be identifiable for the researchers. However, the requirement of anonymity that the researcher observes - just as a medical practitioner does - ensures that participants can remain anonymous as far as possible.

\section{Legal age of consent}

For medical research involving minors, the WMO follows the 'no, unless' principle: Article 4 stipulates that such research is prohibited unless it may be personally beneficial to the minor involved. This type of research is often referred to as therapeutic research. The research is further prohibited if the underage subject is opposed to the research or parts thereof. Article 6 of the WMO regulates the consent requirement for minors: inclusion of minors under the age of 12 is prohibited without the prior written consent of the parents or guardian, and inclusion of minors aged between 12 and 18 is prohibited without the prior written consent of both the minor and his/her parents or guardian. The lower threshold of 12 years is comparable to those in other laws regulating patients' rights, such as the WGBO and the Organ Donation Act (WOD). However, the WGBO puts the upper threshold not at 18 but at 16 . The Doek Commission, which evaluated the WMO, called for the upper age threshold in the WMO to be identical to that in the WGBO [11]. 
In our view, the WMO and the WGBO could be aligned even more closely. If a minor aged 16 years or older wants a medical treatment that the doctor is willing to provide, the treatment can go ahead even in the absence of parental consent. Further, if the minor does not want the parents to be informed, the doctor must respect this wish. If this were to apply not only to medical treatment but also to medical research, evaluation research into medical treatments would face far fewer problems. At present, an individual aged 16 to 18 can give personal consent for a medical treatment, yet needs the permission of both parents for the accompanying research. This jeopardises not only the anonymity but also the autonomy of the minor. As a result, patients in this age group are notoriously difficult to engage in evaluation research.

Even if the consent required for evaluation research among underage patients were brought into line with that for medical treatment, the stipulation 'on paper' would remain problematic. Consider interventions focused on the children of addicted or mentally ill parents, or on helplines such as the General Helpline for Child Abuse (AMK). Gaining parental consent to participate in research on the effects or costs of such interventions is virtually impossible.

\section{Conclusion}

The restrictive interpretation of the term 'written consent' in the WMO hampers the performance of e-research in the Netherlands. For minors, there is the additional problem that both parents also need to give consent. This makes research on sensitive online interventions among young people, for example with regard to problematic parents or child abuse, practically impossible. With their anonymity compromised, young people will simply refrain from participating in research.

These problems can largely be solved by way of two interventions. First, consent given online must be recognised as equal to written consent, as is already the case in other segments of society. Second, the legal age of consent for medical research must be reduced to 16 years, in line with the age of consent for medical treatment. This would allow young people aged 16 and older to make independent decisions on participating in e-research, and enable them to remain anonymous. In this way, the lawmakers and welfare organisations can move with the times. 


\section{REFERENCES}

1. Riper H, Smit F, van der Zanden R, Conijn B, Kramer J, Mutsaers K. High tech, high touch, high trust: Programmeringsstudie E-Mental Health. Utrecht: Trimbos-instituut; 2007.

2. Woolderink M, Smit F, van der Zanden R, et al. Design of an internetbased health economic evaluation of a preventive group-intervention for children of parents with mental illness or substance use disorders. BMC Public Health. 2010;10:470.

3. Wet medisch-wetenschappelijk onderzoek met mensen. Stb. 1998, 161, laatstelijk gewijzigd in Stb. 2013, 306. http://wetten.overheid.nl/ BWBR0009408/geldigheidsdatum_19-11-2013.

4. Dute JCJ. De reikwijdte van de Wet medisch-wetenschappelijk onderzoek met mensen. Tijdschrift voor Gezondheidsrecht 2009;33:427-37.

5. Burgerlijk Wetboek Boek 6, artikel 227a. Stb. 1980, 432, laatstelijk gewijzigd in Stb. $2012,647$. http://wetten.overheid.nl/BWBR0005289/Boek6/Titel5/Afdeling2/Artikel227a/geldigheidsdatum_2011-2013.

6. Besluit METC, Maastricht, afgegeven dd. 20 juli 2011, dossiernummer NL31321.068.10, METC-nummer 10-3-025.

7. Besluit CCMO, Den Haag, afgegeven dd. 20 maart 2012 (besluit hoorzitting, van januari 2012).

8. CCMO/Ministerie van VWS. Medisch-wetenschappelijk onderzoek: Algemene informatie voor de proefpersoon. Den Haag: Ministerie van VWS; 2008.

9. Hooghiemstra TFM. Privacy bij wetenschappelijk onderzoek en statistiek: Kader voor een gedragscode. Den Haag: College bescherming persoonsgegevens; 2002.

10. Federatie Medisch Wetenschappelijke Verenigingen. Gedragscode gezondheidszorgonderzoek. Den Haag/Rotterdam: Medlawconsult/ Stichting FMWK, 2004.

11. Commissie Doek. Advies medisch-wetenschappelijk onderzoek met kinderen. Den Haag: Ministerie van VWS; 2009. 


\section{CHAPTER 7}

General discussion

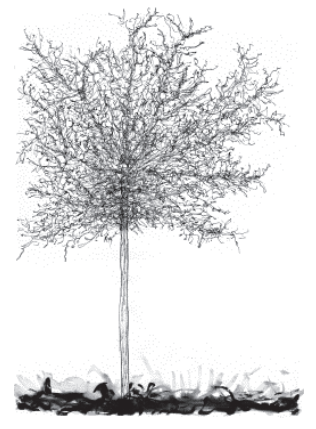



This thesis aims to evaluate several aspects of Kopstoring, one of the first and most frequently offered standardized online courses for adolescents (16-25 years old) dealing with parental mental illness and addiction. Since 2006, the Kopstoring course has been offered online, on www.Kopstoring.nl. It aims to improve the skills of adolescents for coping with the above parental problems. Besides skills training, an objective of the course is to educate adolescents about mental illnesses and addictions and the consequences thereof. The Kopstoring course is provided entirely online; it was initially provided by 9 mental health institutions based in the Netherlands.

In this study, several aspects of this online preventive programme are evaluated: clinical effectiveness, participants' and providers' perspectives, methodology of service use measurement and the. ethical and judicial aspects of online programmes and online scientific studies. The first part of the thesis evaluates the clinical effectiveness of Kopstoring and looks at the content of the programme and the process of delivery. The second part focuses on legal aspects related to provision of online prevention interventions. In addition, more general issues related to service use assessment for youth in need of mental health care are explored in this part.

\section{Main findings: exposing the gap}

From the perspectives of participants and providers, Kopstoring has proven to be a valuable alternative to similar face-to-face interventions, especially for adolescents. This group already experiences a lot of life changes in the transition to adulthood [1, 2]. The anonymous character of Kopstoring is therefore considered very valuable, pragmatic and safe. Being well informed about mental illness and addiction helps with dealing with parental mental illness or addiction problems at home and with improving the relationship with the parents. Peer contact appears to be very valuable as many adolescents dealing with parental mental illness think they are the only ones experiencing it because it is not a topic adolescents talk openly about with their support system or in their everyday environment. In addition, the fact that Kopstoring is provided for free was a big advantage for the participants and was emphasized as very positive by the participants in the process evaluation.

The positive results from the process evaluation were not endorsed in the clinical effectiveness study. The results of the latter study showed a slight improvement in effect, but these were proven to be statistically non-significant. No significant differences were found in effect scores of internalising and externalising symptoms and depression problems between the group that started the course immediately and the group that started the course after 6 months.

Methodological issues arose around topics of measuring and valuating costs related to the adoption of prevention programmes and services used by adolescents in need of mental health care. Speaking to experts in the field of children and adolescent mental 
health and service utilization confirmed the feeling of distance between theory and practice and the existing problems for research.

In addition, problems related to medical ethics and medical law were extremely challenging. The requirements described in Chapter 6, such as having respondents return signed informed consent forms in paper format for online research, make assessments like these included in this study virtually impossible. The consequences of the afore mentioned requirement were devastating for the Kopstoring study. Medical acts and legislation need to be in place to protect the patients or respondents who participate in scientific studies; however, these acts need to be up to date and aid the patient in their participation rather than create obstacles

\section{Interpretation of the main findings}

The outcomes of the clinical effectiveness of Kopstoring speak for themselves; however, they need to be interpreted with caution due to factors such as major organisational restructuring of the youth mental health care system [3] and the mentioned tension between medical ethics and current practice in medical or health-related research. Both of these factors influenced the planning and execution of the randomised controlled trial (RCT) as well as the outcomes of the clinical evaluation, and it is in this light that we need interpret the outcomes of this evaluation of Kopstoring.

Medical ethics: online versus written informed consent

Medical research ethics committees (MRECs) are tasked with judging whether research is practised in conformity with the Dutch Medical Research Involving Human Subjects Act (WMO) and international ethical codes and guidelines. As described in this thesis, Article 6 of the WMO dictates 'written informed consent' for all scientific studies, and this is one of the articles subject to judgement by the MRECs.

To make our case for online informed consent for study inclusion, the regional MREC denied our request to obtain online informed consent; however, they confirmed that the WMO is outdated and therefore impractical to use in certain fields of scientific research. Despite this realisation, the specific MREC also claimed that they are not the right committee to amend the WMO, or any medical act in the Netherlands, because they do not have legislative power. Therefore, MRECs have to deny any deviation from what is written in the WMO, and they do not allow any room for interpretation of provisions in medical acts. The Central Committee on Research Involving Human Subjects (CCMO), the National committee dealing with disputes over medical ethical problems, agrees with the interpretation of the MREC that written informed consent in paper format is the binding requirement for scientific research and that no exceptions should be made.

Because online health care programmes are on the rise [4], evaluation studies of these programmes are becoming more and more common. These evaluation studies are by 
definition non-invasive and use online questionnaires to evaluate programme (cost-) effectiveness. The only reason these studies might be considered subject to judgement by medical research ethical committees in the first place is the fact that respondents are randomly allocated to an intervention or a control condition. With random allocation the natural course of events is interfered with, and that is the reason why studies are subject to the WMO. The current approach for scientists performing evaluation studies of online interventions (with online consent procedures) is to try to follow standard practice as closely as possible and avoid introducing a totally different inclusion procedure for the study as compared to the procedure used in the intervention.

The consequences of this gap between medical ethics and current practice are illustrated in Box 1 by using the Kopstoring randomised controlled trial as an example.

Textbox 1: Consequences of consent procedure

After screening 299 adolescents for eligibility for the Kopstoring course and study, 226 adolescents were eligible for participation and gave online consent. They were first asked for online consent, after which written consent also needed to be obtained. Out of 226 participants who gave online informed consent for participation in the Kopstoring course as well as the study, only 104 (46\%) returned their written informed consent papers. That is a loss of 122 (54\%) potential participants. This problem most likely introduced selection bias to our study. Participants who were not willing to receive a postal package at home (where they likely live with their parents), because it would harm their privacy, refrained from sending their written informed consent.

\section{Medical ethics: legal age of consent}

According to the Dutch Medical Treatment Contracts Act (WGBO), a patient is considered a minor, and therefore not allowed to legally consent to treatment, if a patient is younger than 16 years. At the same time, according to the WMO, for scientific studies evaluating these medical treatments or interventions, the patient needs to be 18 years or older to give legally binding consent. For researchers evaluating interventions for adolescents or the young adult population, and the youngster who is 16- and 17-yearold, are positioned in a very complex situation. This disparity in legal age between these two important medical acts is not only confusing but might also introduce selection bias in scientific studies assessing (clinical) treatments or interventions. In addition, it makes the prospect of including participants younger than 18 years unattractive or even impossible for researchers.

Textbox 2: Consequences of disparity in legal age of consent

In case of the Kopstoring randomised controlled trial, the problem of the disparity in legal age between the WGBO and the WMO most likely led to an underrepresentation of 16- and 17-year-old participants. This group is able to give legally binding consent for the Kopstoring course (treatment/care) but unable to provide legally binding consent themselves for participation in the Kopstoring study. 
Medical ethics: vulnerable populations and their privacy

Following from the previous point, participants in scientific studies under the age of 18 years are obliged to ask for informed parental consent. The WMO states that written informed consent of both parents is required. In case of the Kopstoring course, 16- and 17-year-old participants could enter the course without any problem after giving (informed) consent, but for the study assessing the possible effects of the course, they not only have to inform their parents (which violates their privacy), they also need to get their parents' consent to participate in the study. This requirement puts the adolescent in the difficult position of asking for parental consent for an evaluation study on the effects of a treatment focussing on alleviating the consequences of their parent's mental illness or addiction. The problem of parental consent when dealing with a vulnerable population of people younger than 18 years old has also appeared in interventions focussing on sexual counselling [5] of young adults or interventions related to treating or reducing child abuse and neglect (e.g. child abuse helplines) [6]. In addition, getting both parents to sign parental consent forms is in some cases practically impossible due to the complex situations families are in because of parental mental illness or addiction.

Textbox 3: Consequences of obtaining parental consent in vulnerable populations

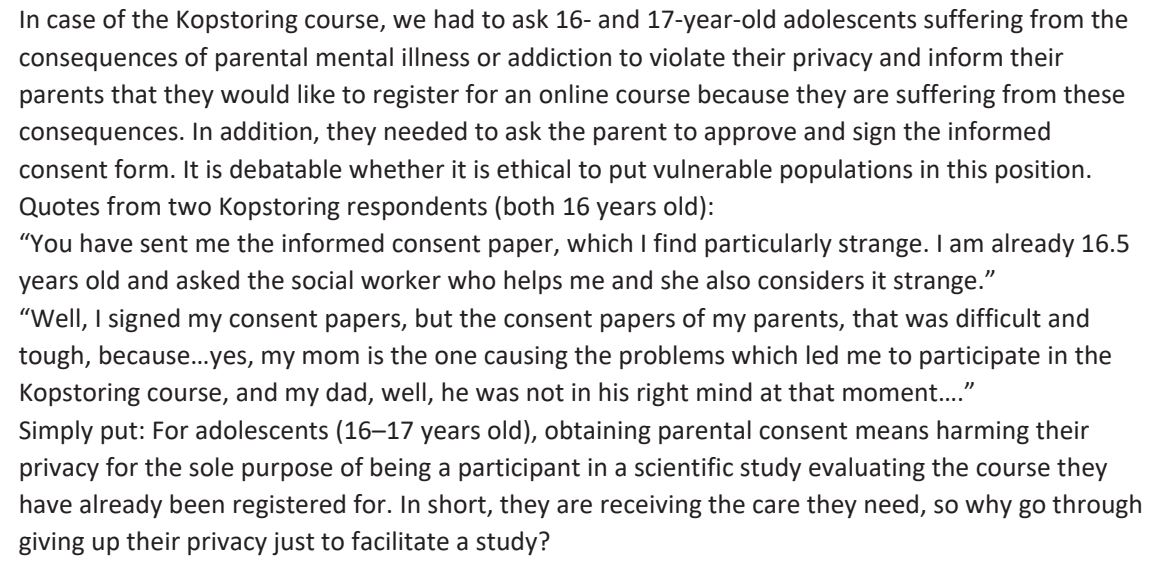

\section{Organisational restructuring: transitions in youth and child care}

The evaluation of Kopstoring took place in a period of economic crisis. In 2015, the Dutch parliament implemented a new organisational and financial structure for youth services in the mental health care system [3]. One of the major consequences of this new decentralised structure was the shift in the authority responsible for financial resource allocation related to mental health care for children and youth. The regional municipalities are now responsible for child and youth (health) care services and child protection services. This change has had severe consequences for the mental health 
institutions providing services for youth such as Kopstoring. Due to this development, entire prevention departments have been declared redundant and professionals have been let go. Managers of mental health organisations see themselves as forced to make decisions about allocation of resources and other financial matters, and in this case the first departments to suffer the consequences were prevention departments.

Textbox 4: Consequences of organisational and financial restructuring in the youth mental health care sector

During the randomised controlled trial, Kopstoring was provided by two professionals from each of a total of nine mental health institutions at the start of the project. Four of these institutions belong to the original core Kopstoring group (which designs and provides courses), and the other five were recruited for the provision of Kopstoring during the trial period. Following the budget cuts and reorganisation of the youth mental health care sector, only six institutions remained. After the RCT inclusion period, only four institutions had found funding to continue provision of the Kopstoring course. Due to the shift in responsibilities from mental health institutions to municipalities, funding for national online (anonymous) interventions has become problematic. Mental health institutions can hardly find money in their local municipalities to cover the costs of providing Kopstoring because the municipalities are not eager or willing to pay for online health care for participants who could be outside their region, and health insurers need personal information to be able to cover the costs (and it is an anonymous course).

For the Kopstoring RCT, the team of professionals has changed several times. Providers have had difficulties convincing management of the worth of continuing to provide Kopstoring. Some providers and some mental health institutions have not been able to keep their legal commitment to Kopstoring due to the aforementioned changes in financial and organisational structure. These changes might have influenced the decision of whether to keep Kopstoring.

\section{Screening and inclusion problems:}

In addition to the medical ethical and organisational problems the Kopstoring trial encountered practical problems during the execution of the trial, such as the fact that the population showed more clinical symptoms than was to be expected in a prevention setting. The consequence of the latter was the exclusion of potential participants who, in clinical practice, would have been perfectly eligible for the course. The combination of these factors necessitated a number of modifications (see box 5), and therefore a deviation from the initial protocol. 
Textbox 5: Overview of changes to the initial design of the RCT

After start of the trial, a number of modifications in the trial design [7] were implemented. The changes concerned the power calculation, the screening procedure (exclusion criteria) and the choice of secondary outcome measures.

The target population showed more clinical symptoms than was to be expected in a prevention setting. The consequence of the latter was the exclusion of potential participants who, in clinical practice, would have been perfectly eligible for the course. The unfortunate choice of the Youth Self Report as a screening instrument led to a deviation from the original screening procedure. The Effect Evaluatie Lijst (EEVL) was used as screening instrument instead of the Youth Self Report and complemented with additional questions to be assessed by the professionals from the Mental Health Services. In addition, the a-priori choice of using the Symptom Checklist-90 (SCL-90) as secondary outcome measure appeared to be ill-suited. The SCL-90 was too long and captured too many of the same concepts as the YSR. Therefore, the Centre for Epidemiologic Studies-Depression

Scale (CES-D) was used instead, which is a much shorter instrument and focuses on capturing internalising problems. In addition, the recruitment problems and the availability of new information about the correlation of YSR measures, were reasons to reconsider the (conservative) power calculation.

Weighing the combined impact of these factors illustrates their severity and possible effects on the results of the evaluation of Kopstoring. In this light, one may argue that the study results could have been different if the legal, financial and organisational context had been more facilitating. The process of enrolment, screening and data collection of the evaluation study in this thesis were very dependent on the context described above.

\section{Limitations and strengths: minding the gap}

\section{Limitations}

The major limitations of the particular studies included in this thesis were addressed in the previous section and in the several chapters. In this section, some general limitations of the studies performed will be discussed.

Firstly, the severity of symptoms of the participants was underestimated. The Kopstoring course is a preventive course and aims to aid adolescents in coping with parental mental illness and addiction. We therefore aimed to exclude adolescents who had been diagnosed with a mental illness or addiction, in the screening process. In addition, we used the Youth Self Report (YSR) internalising scales as a screening instrument. We found that the vast majority of potential participants scored higher than the cut-off scores (male: 16, female: 21) [8] on the internalising YSR scales. Higher scores mean more complaints and therefore a worse health state. Because of these high scores, we had to conclude that almost all candidates were ineligible to participate in the trial. Changing the screening procedure resulted in a population with moderate to severe clinical complaints, while it was expected that symptoms in the study population would be largely subclinical. This raises the question whether the Kopstoring 
course, with its preventive nature, is 'intense' enough to help the adolescent offspring of parents with mental health or addiction problems. A mismatch between course and population may have caused the absence of any effect.

Secondly, another limitation was the random allocation procedure, which may have been biased because of the requirement to obtain written informed consent. Random allocation took place after online informed consent, but before written informed consent. As a consequence, participants who were allocated to the waiting list condition may have been less motivated to return their written informed consent. Also, one could imagine that those in the waiting list group that experienced more problems were particularly reluctant to return the consent forms. This is what probably caused the imbalance in the groups at baseline.

Thirdly, data collection for the studies in this thesis always used the adolescent as the source of information. This means that all data were collected from the participants directly through self-reported assessments. Consequently, the data were based on the self-reported information of individual participants, and it might have been preferable to have more sources to validate this information [9-11].

A fourth limitation lies in the technical sphere. With the rise of online interventions and evaluations in health care, more interventions are being developed for use via computer and mobile phone applications. These developments might be advantageous in many cases; however, they are also prone to introducing technical failures. In the Kopstoring study, we had some technical failures, some more severe or harder to solve than others.

A possible limitation of measuring short-term effects is that the intervention might have an effect over a longer period of time, which is a very likely phenomenon within the field of prevention. The potential delayed effect of the intervention, also described as the sleeper effect [12], could not be ruled out, and effects of the intervention measured at 12 or 24 months might have shown different results. 


\section{Strengths}

Despite both major and minor limitations, this thesis contains study results that shed new light on online health care and online evaluation research. The Kopstoring randomized controlled trial is the first study to assess the clinical effectiveness, and participants' and providers' perspectives of an online preventive course for adolescent children of parents with mental health or addiction problems.

The study's most essential strength follows from its most essential limitation. This thesis contains material exploring and describing a very real problem in Dutch medical research ethics. It is the first of its kind to create awareness of the gap between actual regulation and what is done by professionals in the field. Not only are medical acts narrowly interpreted and unable to grow alongside the technological advances of this era, but even the MRECs and the Central Committee on Research Involving Human Subjects (CCMO) admit that this is a real and large problem. However, these committees cannot amend legal acts or regulations and refrain from interpreting them less narrowly in order to keep the division of tasks separated; they instead merely check studies' protocols to judge whether or not these protocols are in line with medical ethical legislation made by parliament. Our study has shed some light on the size and severity of this problem, and hopefully the problem will be noticed by politicians and parliament, the ones with legislative power.

Secondly, this study focusses on an intervention for a very vulnerable group. Not much research has been done exposing the risks and consequences for children of parents with mental illness or addiction problems. However, more evidence of these risks and consequences has slowly become available. The number of interventions for these atrisk children is growing; most of them are family oriented or focus on the patient (the parent in this case). Kopstoring has been evaluated as very good by the participants themselves, giving them a voice in this process as well. With the knowledge gained from the included studies, we are now able to provide more information about this target population.

Kopstoring has been noticed internationally by research teams from Sweden and the United States. Despite the fact that the evaluation used in this thesis showed no statistically significant effects of Kopstoring, the course has been deemed effective and helpful enough that at the moment a team in Sweden has translated the material and is performing a pilot study in preparation for a multi-centre randomized controlled trial.

\section{Recommendations: bridging the gap}

In total, 17 recommendations can be given based on the outcomes of the studies in this thesis. Due to the variety of factors assessed in this thesis, recommendations can be divided into two categories: recommendations for policy- and decision makers and recommendations for future (online) research. 


\section{Recommendations for policy-and decisions makers}

- Firstly, we recommend starting a political discussion about the increasing problems and gap caused by growing medical and other technologies in this digital era and by outdated rules and regulations.

- Secondly, we recommend that consent given online be recognised as equal to written consent, as is already the case in other segments of society in the Netherlands.

- Thirdly, we recommend reducing the legal age of consent for medical research to 16 years, in line with the age of consent for medical treatment. This would allow young people aged 16 and older to make independent decisions on participating in e-research, without violating their privacy.

- As a fourth recommendation, we advise that the tasks of the Medical I Research and Ethics Committee (MREC) and the Central Committee on Research Involving Human Subjects (CCMO) be expanded and not restricted to merely judging whether research protocols are within the limits of good conduct and obedience to medical ethical law. However, if the committees conclude that a research team might have a valid and good point proving the law to be outdated, they should be able to institute an exemption rule or a procedure to suggest changes to the current medical act to parliament, using the case at hand.

- Our fifth recommendation is to use a multilevel approach to address the problems of children dealing with parental mental illness or addiction. The problems of this group of children need to be more widely recognized in Dutch society.

\section{Recommendations for further research}

- Firstly, we recommend that future clinical studies evaluating similar preventive interventions for youth include coping mechanisms as an intermediate outcome measure alongside the measured clinical outcomes, as coping has proven to be a good tool for adolescents dealing with parental mental illness or addiction [13].

- Secondly, we recommend to avoid any situation in which randomization takes places based on online informed consent, when awaiting written informed consent. This might lead to unbalanced groups at baseline.

- Thirdly, we recommend using a participatory approach in future studies. Evidence has shown $[14,15]$ that this is very effective for understanding and empowering the target population. Including persons from the target population as part of the study group empowers them to direct the research team to include aspects important for research and outcomes. 
- In addition we recommend more qualitative studies on the experience and importance of anonymous online interventions. The process evaluation included in this thesis gave a full understanding of experiences and expectations of participants in Kopstoring. It showed the importance of aspects that would not have been discovered with merely quantitative research.

- Then, we recommend thinking thoroughly about the consequences of some technical gaps that have not been overcome yet in online preventive research (e.g. participants who register a second time when allocated to the waiting list)

- A sixth recommendation for future research is seeking a very close collaboration between the research team and the providers, the health professionals. Because of the virtual nature of online studies, it can be hard to keep everyone together. Research teams have to be invested in goodwill and avoid an ivory tower attitude.

- A seventh recommendation for future research is to double-check and monitor the process several times. Despite the fact that systems are online and researchers rely on programs to alert them when something goes wrong, it is often noticed too late. During the inclusion period of a randomized controlled trial, we recommend a data monitoring system, but a person acting as a monitor is preferable.

- Another recommendation, is to compare Kopstoring to a different comparator, e.g. a face-to-face equivalent, when assessing cost effectiveness. Care as usual effectuated with a waiting list control group already seems to result in lower costs compared to the provision of Kopstoring.

- Our ninth recommendation is to include measures other than self-reported ones, such as databases or registries, to validate service utilization.

- We also recommend starting to explore methods of valuating school absenteeism in at-risk offspring of parents with mental illness or addiction. Children of parents with mental illness or addiction are at an elevated risk of developing these problems themselves. In addition, they are very likely to be informal caregivers. This may be provoking (regular) school absenteeism.

- In accordance with the previous recommendation, we also recommend looking at the impact of presenteeism in school settings. For work settings, this phenomenon has been explored and described, and valuation methods exist. For school settings, it has not been explored in depth, which may be having a considerable impact on adolescents as well as society.

- For online research, we recommend making sure to follow the latest updates and technical innovations of programmes. Nowadays, web-based interven- 
tions can be accessed with several devices as well as on the go. Researchers should make sure they have compatible programmes and make completing the questionnaires as easy as possible for the participants.

- Finally, we recommend to improve understanding and uniformity in definitions for mental health services in the field (uniformity advances service use measurement and therefore provides accurate input for the cost side of economic evaluations); this could aid future studies in collecting service use data and make results more comparable and uniform.

\section{Concluding remarks}

In conclusion, the studies presented in this thesis have shown results that should be taken into account by decision- and policy makers as well as researchers. Despite the lack of statistical significant evidence for the effectiveness of Kopstoring, the results of the process evaluation show the potential of Kopstoring and stress the need for further research into the potential of online preventive interventions. In addition, some study results in this thesis emphasise the pressing need to discuss the gaps between (bio)medical research, policy framework, and legal acts. 


\section{REFERENCES}

1. van Loon L. Adolescents and Parental Mental Illness- Risk and protective factors for internalizing and externalizing problems. PhD dissertation. Nijmegen: Radboud University Nijmegen; 2015.

2. Costello EJ, Mustillo S, Erkanli A, Keeler G, Angold A. Prevalence and development of psychiatric disorders in childhood and adolescence. Arch Gen Psychiatry. 2003;60(8):837-44.

3. Nederlands Jeugdinstituut, Utrecht 2016 [cited 2016 19-04].

4. Riper, H., Smit, F., van der Zanden, R., Conijn, B., Kramer, J., Mutsaers, K. High Tech, High Touch, High Trust. Programmeringsstudie E-Mental Health. Utrecht: Trimbos Instituut, 2007.

5. Grauvogl A. Let's talk about sex- Sexual health and sexual dysfunction among adolescents. PhD dissertation. Maastricht: Maastricht University; 2014.

6. Snoeren F. Giving maltreated children a voice- A study of self-reported quality of life, and the effectiveness and cost-effectiveness of the child-interview intervention during the investigation of reports of child maltreatment. PhD dissertation. Maastricht: Maastricht University; 2014.

7. Woolderink M, Smit F, van der Zanden R, Beecham J, Knapp M, Paulus A, et al. Design of an internetbased health economic evaluation of a preventive group-intervention for children of parents with mental illness or substance use disorders. BMC public health. 2010;10:470.

8. Verhulst CF, Ende vd, E., Koot HM. Handleiding voor de youth self-report (YSR). Rotterdam EU, editor. Rotterdam 1997.

9. Farmer E, Angold A, Burns B, Costello E. Reliability of Self Reported Service Use: Test-Retest Consistency of Children's Responses to the Child and Adolescent Service Assessment (CASA) Journal of Child and Family Studies. 1994;3(3):18.

10. Theunissen N, Vogels T, Koopman H, Verrips G, Zwinderman K, Verloove-Vanhorick S, et al. The proxy problem: child versus parent report in health-related quality of life research. Quality of life research 1998;7:10.

11. van den Brink M, Van den Hout W, Stiggelbout A, Van de Velde J, Kievit J. Cost Measurement in Economic Evaluations of Health Care, Whom to ask? Medical Care. 2004;42(8):740-7.

12. Kumkale GT, Albarracin D. The sleeper effect in persuasion: a meta-analytic review. Psychological Bulletin. 2004;130(1):143-72.

13. van Veen C, Van der Zanden R. Procesevaluatie Kopstoring; Een onderzoek naar de preventieve online groepscursus voor jongeren van 16-25 jaar Utrecht: Trimbos Instituut, 2007.

14. Bindels J, Baur V, Cox K, Heijing S, Abma T. Older people as co-researchers: a collaborative journey. Ageing \& Society. 2014;34(06):951-73.

15. Abma TA, Nierse CJ, Widdershoven GA. Patients as partners in responsive research: methodological notions for collaborations in mixed research teams. Qualitative health research. 2009;19(3):401-15. 
SUMMARY 

This thesis reports the outcome of an evaluation of an online preventive course for adolescent children of parents dealing with mental illness or addiction. The evaluation integrates the clinical perspective, the perspectives and experiences of patients and providers, the judicial and ethical perspectives and the service utilisation perspective. The data for this thesis was collected by means of a randomised controlled trial. Additional data were collected for a study evaluating methodological issues with the service utilisation of youth in need of mental health care. The first step of the study entailed the recruitment and enrolment of the offspring (in the 16-to-25-year age group) of people with psychological or addiction problems. Maastricht University in close collaboration with the Trimbos Institute (the Netherlands Institute of Mental Health and Addiction) and nine mental health institutions ${ }^{3}$ carried out the recruitment. Several online and offline recruitment strategies were used to reach the target population. After recruitment, enrolment took place, and professionals from the nine mental health institutions carried out screening. The second step was the random allocation of the participants to either the Kopstoring course or a six-month waiting list control group. After these steps, baseline and follow-up measurements were taken to collect data for the research in this thesis.

The first part of this thesis includes chapters that report the analysis of the data collected as outlined.

Chapter 1 discusses the relevance of the thesis, its aims and objectives and the methodologies used. The severity and size of the problem is most likely underestimated in the Netherlands, and the prevalence of children dealing with parental mental illness is probably high. It is important to address the problem's size in addition to its nature. Studies have repeatedly shown that a child who has been exposed to a parental psychiatric disorder or addiction is at increased risk (genetic, biological and psychological) of developing the same (or a comparable) condition. Interventions for this at risk group have been developed. However, most of them have a family focus or even focus solely on the patient, that is, the parent. This thesis focuses on an intervention for the adolescent offspring of the patient and includes adolescent children (aged 16-25 years) of patients with mental illness or addiction problems. The aim of the thesis is to evaluate the Kopstoring course from different perspectives. Therefore, we perform a multiperspective evaluation that includes clinical effectiveness, participants' and providers' expectations and experiences, judicial and ethical perspectives and methodological perspectives.

\footnotetext{
${ }^{3}$ GGNet, Mindfit (until recently, Dimence), GGZ Oost Brabant, Ypse (until recently, Reinier van Arkel), VVGI (until recently, GGZ NML) \& Riagg Zuid, Context, Brijder, Eleos (until recently, Optiment)
} 
Chapter 2 describes the content of the online preventive Kopstoring course and its components and structure. In addition, it describes the methodology used to collect data.

Chapter 3 reports the process evaluation of Kopstoring and focuses on the expectations and experiences of the participants and providers. Data were collected by means of in-depth interviews with 13 participants and 4 providers. Analyses of these interviews revealed that, in general, participants' experiences with the Kopstoring course were very positive and met their (a priori) expectations. At a deeper level, analysis uncovered 5 main themes: background, requirements for the intervention, experience with the intervention, technical aspects and research-related aspects. The background revealed how the participants heard about Kopstoring and information regarding their personal situations. The second theme reflected the requirements for participation (e.g. Internet, preparation of the course etc.), and the third theme described the experiences of the participants and providers with the online intervention. The participants provided information about the content of the sessions, the quality of the provision and the importance of peer support. The fourth theme yielded information about the technical aspects of the website and the chat box (e.g. colours, fonts and errors) and the fifth theme encompassed research components such as the waiting list, randomisation and the discussion of consent in depth. Overall, the participants' experience with Kopstoring was extremely positive, and they considered it a highly valuable intervention. In addition, they appreciated its online aspect and valued being anonymous. They also considered privacy to be a key component of Kopstoring. Most of the barriers that were identified during the in-depth interviews were related to technical aspects of the course, for example, loss of connectivity. Other barriers existed with regard to the randomised controlled trial. They were, namely, the gathering of (written) informed consent and that of parental consent in the case of minors. The process evaluation also included the experiences of the course providers. The providers felt comfortable and confident providing Kopstoring online. They emphasised the quality of the course and the quality of the protocol. A barrier for the providers was participation in the randomised controlled trial. They reflected on its limitations (randomisation, screening procedure and inclusion and exclusion criteria). The providers also suggested ideas for the improvement of the Kopstoring course, including more input from them in the decision making process.

Chapter $\mathbf{4}$ assesses the clinical effectiveness of the online Kopstoring course. This study compared the participants in the Kopstoring course with a six-month waiting list control group. The results suggest that there are no statistically significant differences between the intervention and control groups' internalisation and externalisation of problems and depression symptoms. The inclusion of the target population was problematic due to the legal obligation to gather written informed consent in the online context. Written informed consent forms were not easily obtained, and, therefore, the sample size remained small and the trial underpowered. Most likely, these problems 
also introduced selection bias into the trial. In this light, the results of the clinical evaluation study should be interpreted with the utmost care.

Chapters 5 and 6 focus on the problems encountered with data collection and the analysis of the Kopstoring randomised controlled trial. More specifically, chapter $\mathbf{5}$ describes the burden of mental illness and how economic evaluations can help decision makers deal with the allocation of scarce resources, as is the case in the health care sector. In order for economic evaluations to optimally inform decision makers and policy makers, the accurate measurement of service use is critical. Such measurement is the basis for the cost calculation aspect of the economic evaluation. Based on expert interviews, this qualitative study identifies the best approaches to service use measurement for child mental health conditions. In addition, it identifies and describes problems in the current theory and methodology. The results suggest considerable expert agreement on the strengths (e.g. the availability of accurate instruments to measure service use) and weaknesses (e.g. the lack of unit prices for services outside the health care sector or alternative approaches to service use measurement). Experts also identified some unresolved problems, for example, the lack of uniform definitions for some mental health services. This problem implied that different approaches to the provision of mental health services were being compared because they had the same definition or name. In fact, they were not comparable because their content was not uniform. The study had an international outlook and, therefore, experts from the United Kingdom, the United States of America and the Netherlands were interviewed.

Chapter 6 describes a fundamental problem experienced with the Dutch Medical Research Involving Human Subjects Act (WMO). This act requires the written consent of participants involved in medical research, making online research unnecessarily complicated and, at times, impossible. A great deal of e-research involves online interventions focusing on sensitive issues (e.g. sexual dysfunction, addiction and child abuse). The assurance of anonymity is an important precondition for participation in this type of e-intervention and its evaluation in e-research. However, the word 'written' in WMO protocols is strictly interpreted to mean 'on paper', and participants are not permitted to give consent through other means (e.g. email). A second requirement, which complicates research involving minors aged 12 to 17 years in particular, is that both parents or the guardian of each child give written consent. This undermines the principle of privacy to such an extent that finding participants is virtually impossible. While participants in medical research must be identifiable to the researcher, their anonymity must otherwise be guaranteed as far as possible. In this study, we focus on the idea of amending the Dutch WMO. In addition, this study describes and explores the differences between the legal age of decision making and consent for medical research and for participation in e-research. For treatment, a person aged 16 years or more can make legally binding decisions (Medical Treatment Contracts Act, WGBO), while, for scientific studies (including the evaluation studies for these treatments), solely a person aged 18 years or more can make legal decisions. The inconsistencies 
between the two most highly used medical acts (the Medical Treatment Contracts Act (WGBO) and the Dutch Medical Research Involving Human Subjects Act (WMO)) regarding the age at which one can provide legally binding consent cause problems in the assessment of interventions.

Chapter 7 summarises the main findings of the studies that this thesis describes. In addition, it interprets the findings and reflects on the strengths and limitations of the studies. The outcome measures chosen for the analysis do not reflect the positive effects and the experiences of both the providers of and participants in Kopstoring (i.e. they are statistically unconfirmed). However, we need to interpret the results with the utmost caution due to all obstacles encountered during the Kopstoring study. This chapter also formulates recommendations for policymakers and decision makers and for future research. Examples of the recommendations for policymakers and decisions makers include starting a political discussion about increasing problems and gaps between the rules and regulations and current practice. We go on to recommend that consent given online be recognised as being equal to written consent. For future research, we recommend including coping mechanisms as an intermediate outcome measure alongside clinical measures in clinical trials (as coping has proven to be a good tool for adolescents dealing with parental mental illness) and performing more qualitative studies that look into experiences concerning online anonymous interventions and the importance of the same.

In conclusion, decision makers and policymakers as well as researchers should take into account the results of the studies presented in this thesis. While the effectiveness of Kopstoring has only been confirmed in a process evaluation, the outcomes of the included studies show its potential and stress the need for further research into the power and potential of online preventive interventions. In addition, some of the study results of this thesis address the pressing need to discuss the gaps between (bio)medical research and the policy framework, structure and legal acts. More than ever, we should be aware of the problems hampering the provision of online health care and the evaluation of the same. 
SAMENVATTING 

Dit proefschrift beschrijft de evaluatie van een online preventieve cursus voor jongvolwassen kinderen van ouders met psychische- of verslavingsproblemen (in Nederland ook wel KOPP/KVO kinderen genoemd). Deze evaluatie integreert verschillende perspectieven waaronder het klinische perspectief, patiënt en uitvoerders perspectief in vorm van verwachtingen en ervaringen, het juridische- en ethische perspectief en het zorggebruik (kosten) perspectief. De data waarop de resultaten van dit onderzoek is gebaseerd, is verzameld met behulp van een gerandomiseerd multi-centrum experiment. Additionele data is verzameld voor de studie die methodologische problemen van zorggebruik van kinderen en jongeren in de geestelijke gezondheidszorg evalueert. De eerste stap in het datacollectie proces was de rekrutering en inschrijving van jongvolwassen kinderen (16-25 jaar oud) van ouders met psychische- of verslavingsproblemen. De rekrutering en PR werd geleid door het Trimbos Instituut (in nauwe samenwerking met Universiteit Maastricht). Voor de rekrutering zijn zowel online als offline strategieën toegepast om de doelpopulatie te bereiken. Na rekrutering en inschrijving zijn de potentiele deelnemers gescreend door getrainde professionals van negen GGZ-instellingen ${ }^{4}$, verdeeld over Nederland. Stap twee was de toewijzing van de gescreende deelnemers in ofwel de Kopstoring groep ofwel de wachtlijst groep. Na het doorlopen van dit proces hebben een aantal metingen plaatsgevonden en zijn de Kopstoring cursussen uitgevoerd.

Het eerste gedeelte van dit proefschrift bevat hoofdstukken die resultaten beschrijven gebaseerd op analyses van data van het hierboven beschreven Kopstoring experiment.

Hoofdstuk 1 beschrijft de relevantie van dit proefschrift alsmede de hoofdvraag en doelen. Hoofdstuk 1 gaat tevens in op de toegepaste methodologie, die gebruikt is om tot beantwoording van de vraagstelling te komen. De ernst en omvang van de problemen die KOPP/KVO kinderen hebben, wordt onderschat in Nederland en de prevalentie van kinderen met een ouder met psychische- of verslavingsproblematiek is hoog. Naast de ernst en de omvang van het probleem is het belangrijk om aandacht te vestigen op de aard van het probleem. Uit wetenschappelijke bronnen blijkt namelijk dat een kind (of jongvolwassene), dat blootgesteld wordt aan psychiatrische- of verslavingsproblematiek van de ouder, een verhoogd risico heeft (zowel op genetisch, biologisch als psychisch vlak) om dezelfde problemen of ziekten te ontwikkelen als de ouder. Interventies voor deze kwetsbare groep zijn inmiddels ontwikkeld, helaas hebben de meeste interventies de focus op de gehele familie of juist de patiënt alleen (dus de ouder) en wordt het kind vaak vergeten in het proces. Dit proefschrift richt zich mede daarom op een interventie speciaal ontwikkelt voor jongvolwassen kinderen (16-25 jaar oud) van ouders met psychische- of verslavingsproblemen. Het doel van dit proef-

\footnotetext{
${ }^{4}$ GGNet, Mindfit (tot voorkort, Dimence), GGZ Oost Brabant, Ypse (tot voorkort, Reinier van Arkel), VVGI (tot voorkort, GGZ NML) \& Riagg Zuid, Context, Brijder, Eleos (tot voorkort, Optiment)
} 
schrift is het uitvoeren van een evaluatie van de online Kopstoring cursus vanuit verschillende perspectieven. We evalueren daarbij het klinisch effect van Kopstoring, maar ook verwachtingen en ervaringen van patiënten en uitvoerders. Verder zijn ook juridische en ethische perspectieven en methodologische perspectief meegenomen in de evaluatie.

Hoofdstuk 2, beschrijft de inhoud van de Kopstoring cursus en gaat dieper in op de verschillende componenten van de online cursus. In dit hoofdstuk wordt ook dieper in gegaan op de opzet en methodologie van dataverzameling voor de klinische evaluatiestudie van Kopstoring.

Hoofdstuk 3 rapporteert de resultaten van de procesevaluatie van Kopstoring en richt zich op verwachtingen en ervaringen van deelnemers aan de Kopstoring cursus en van de uitvoerders van de cursus. Data voor de proces evaluatie is verzameld door middel van diepte interviews met zowel deelnemers als uitvoerders van de cursus. 13 jongvolwassenen (16-25 jaar oud) en 4 uitvoerders zijn hiervoor geïnterviewd. Analyses van deze diepte interviews lieten zien dat de ervaringen van de deelnemers van de Kopstoring cursus over het algemeen heel positief waren en tevens in lijn met hun verwachtingen. Analyses van de data legde 5 thema's bloot namelijk: de achtergrond van deelnemers en inschrijving, ervaringen met de interventie, technische aspecten van online hulpverlening en Kopstoring, en onderzoeksaspecten. In het algemeen werd de Kopstoring cursus zeer gewaardeerd door de deelnemer alsmede door de uitvoerders. Het eerste thema legde bloot hoe deelnemers bij de Kopstoring cursus terecht kwamen en wat de reden was voor inschrijving en persoonlijke situatie. The tweede thema reflecteerde op de vereiste voor deelname aan de cursus (bijv. internet toegang, voorbereiding van de sessie etc.) en het derde thema beschreef de verwachtingen en ervaringen van de deelnemers en uitvoerders. De deelnemers gaven informatie over de inhoud van de sessies, de kwaliteit van de uitvoer, en de relevantie en belang van lotgenotencontact. Het vierde thema, gaf inzicht in het technische gedeelte van de website en de chatbox (bijv. kleuren, fouten, lettertype, inloggen etc.) het laatste thema gaf inzicht in de ervaringen met de onderzoekscomponenten zoals de wachtlijst, randomisatie en de toestemming voor deelname. Het online aspect van de cursus, dat de privacy en anonimiteit van de deelnemer waarborgt, werd als sleutel component benoemd in de positieve beoordeling van de Kopstoring cursus. Obstakels die ervaren werden door deelnemers, lagen vooral op technisch vlak. Daarnaast werden sommige aspecten van het onderzoek (zoals het risico om op een wachtlijst te komen, het versturen van schriftelijke toestemmingsformulieren, en in geval van minderjarigheid het verkrijgen van ouderlijke toestemming) als barrière gezien. In de procesevaluatie zijn ook data verzameld middels diepte interviews met de uitvoerders van de Kopstoring cursus. Deze data laat zien dat de geïnterviewde uitvoerders (professionals) zich vertrouwd voelen met de inhoud van de cursus en de online uitvoer ervan, ze benadrukken de goede kwaliteit van de inhoud van het draaiboek. De uitvoerders hebben sommige onderzoekscomponenten als obstakels ervaren en vonden dit dan ook een limitatie 
voor uitvoer (randomisatie, de wachttijd, de screeningsprocedure van het onderzoek en de in-en exclusie criteria van het onderzoek). De uitvoerders brachten verder ideeen voor verbetering aan waaronder een nauwere samenwerking van onderzoeksteam en praktijk.

Hoofdstuk 4 evalueert de klinische effecten van de online Kopstoring cursus. In deze studie wordt de Kopstoring cursus vergeleken met een wachtlijst controle conditie, waarin deelnemers gebruik maken van 'normale zorg'. Resultaten laten zien dat er geen statistisch significant verschil wordt gevonden tussen de twee groepen in termen van internaliserende (depressie en angst) en externaliserende symptomen (agressie) en problemen en depressie symptomen. Ondanks het ontbreken van statistische significantie in effect, waren sommige verbeteringen wel zichtbaar. Verschillen tussen de groepen waren zichtbaar op de internaliserende schalen van de Youth Self Report en suggereerde een verbetering van scores in de interventiegroep. Gezien de impact van methodologische, medisch-ethische en juridische problemen en organisatorische problemen dienen de resultaten van deze klinische effectenstudie met grote voorzichtigheid geïnterpreteerd te worden.

Hoofdstuk 5 en 6 richt zich primair op de problemen die we ondervonden hebben tijdens de het verzamelen van de data en met het design en de uitvoer van de Kopstoring studie. Hoofdstuk 5 beschrijft de impact van psychische ziekten en hoe economische evaluaties beleidsmakers kunnen helpen in het nemen van beslissingen over verdeling van schaarse middelen in de gezondheidszorg. Om economische evaluaties zo goed mogelijk tot hen recht te laten komen en zo optimaal mogelijk gebruik te maken van deze evaluaties, is accurate meting van zorggebruik kritiek en onmisbaar. Zorggebruik dient als basis voor het berekenen van de kosten in een economische evaluatiestudie. In dit hoofdstuk is de uitkomst van een kwalitatieve studie beschreven, die gebaseerd is op interviews met experts. In deze diepte interviews, identificeren zij de beste methode en zienswijze om zorggebruik van kinderen (en jong volwassenen) die zorg nodig hebben voor psychische problemen in kaart te brengen. Verder worden de problemen met de gangbare theorieën en methoden geïdentificeerd en beschreven. Analyses laten verder zien dat er goede overeenstemming is tussen experts met betrekking tot de sterke punten (bijv. beschikbaarheid van goede en accurate meetinstrumenten om zorggebruik te meten) en de zwakkere punten (bijv. het ontbreken van uniforme kostprijzen voor diensten buiten het reguliere zorgsysteem, en andere alternatieve methoden voor het meten van zorggebruik). De geïnterviewde experts identificeerde ook enkele problemen, waar zich nog geen eenduidige oplossing voor heeft aangediend, zoals het ontbreken van uniforme definities voor hulpverlening of diensten. Deze kwalitatieve studie had een internationale insteek en daarom zijn er experts van het Verenigd Koninkrijk, Amerika en Nederland geïnterviewd.

Hoofdstuk 6 schetst een fundamenteel probleem met de Nederlandse Wet medischwetenschappelijk onderzoek met mensen (WMO). Deze wet schrijft schriftelijke geïn- 
formeerde toestemming van de deelnemer van medisch-wetenschappelijk onderzoek voor. Dit maakt online onderzoek (e-research) onnodig gecompliceerder en in sommige situaties zelfs praktisch onmogelijk. Een groot deel van online onderzoek betreft het onderzoek naar interventies die gericht zijn op behandeling (of preventie) van sensitieve problemen (bijv. seksueel disfunctioneren, verslaving of kindermishandeling). De waarborg van anonimiteit is bij online behandeling en online onderzoek een belangrijke voorwaarde voor deelname aan dit type onderzoek (en behandeling). Desalniettemin, wordt het woord 'schriftelijk' als opgenomen in de WMO, strikt geïnterpreteerd als 'op papier' en deelnemers zijn daarmee niet bevoegd om toestemming te geven voor online onderzoek, middels elk ander medium (e-mail, scan, vinkje, of klik met de muis). Een tweede vereiste van de WMO, wat (niet invasief) onderzoek onnodig compliceert, is de regelgeving omtrent minderjarigen, vooral de minderjarigen in de leeftijd 12 tot 17 jaar oud. Voor deze groep minderjarigen volstaat hun eigen geïnformeerde toestemming voor deelname aan online onderzoek niet. Hun ouders dienen ook geïnformeerde toestemming te verlenen voor deelname van hun kind aan online onderzoek. Dit ondermijnt de privacy van de deelnemer zo erg, dat rekrutering van deze deelnemers vaak bijna onmogelijk blijkt. De privacy van de deelnemer van online onderzoek dient ten alle tijden gewaarborgd te worden, zelfs met in acht neming dat de deelnemer wel herleidbaar moet zijn voor de onderzoeker. In deze studie stellen wij een wetswijziging voor van de WMO. Ten tweede worden de verschillende leeftijdsgrenzen waarop een persoon geacht wordt juridisch bindende beslissingen te mogen nemen ten aanzien van behandeling (vanaf 16 jaar) en ten aanzien van onderzoek naar deze behandelingen (vanaf 18 jaar) beschreven en vergeleken. Deze inconsistentie in minderjarigheidsgrenzen tussen de wet geneeskundige behandelingsovereenkomst (WGBO) en de wet medisch-wetenschappelijk onderzoek met mensen (WMO) veroorzaakt grote problemen voor de evaluaties van zorgprogramma's behandelingen of interventies.

In hoofdstuk 7 worden de algemene bevindingen van de deelstudies van dit proefschrift samengevat. Naast de interpretatie van de resultaten heeft dit hoofdstuk als doel te reflecteren over de sterke- en zwakke punten van de evaluatie van Kopstoring. De uitkomstmaten, die meegenomen zijn in deze studie, onderschrijven de positieve uitkomsten van de ervaringen van deelnemers en uitvoerders niet, en resultaten blijven daarmee statistisch niet significant. De resultaten dienen echter met voorzichtigheid geïnterpreteerd te worden vanwege de enorme problemen (methodologisch, financieel, organisatorisch, problemen met rekrutering, technische problemen etc.) waar dit onderzoek mee te kampen heeft gehad. Tot slot worden aanbevelingen voor beleidsmakers en onderzoekers gedaan. Beleidsmakers wordt bijvoorbeeld aanbevolen om de politieke discussie op gang te brengen om de minderjarigheidsgrenzen in wetgeving in de gezondheidszorg uniformer te maken en mensen bewust te maken van de problemen die spelen in het onderzoeksveld zodat het gat tussen wetgeving en praktijk kleiner wordt. Ook zou online toestemming mogelijk moeten zijn naast schriftelijke 
toestemming. Voor toekomstig onderzoek adviseren we om coping mechanisme als intermediaire maat mee te nemen in de klinische evaluaties van online interventies voor KOPP/KVO groepen. Tot slot zou er meer kwalitatief onderzoek gedaan moeten worden naar de ervaringen met online anonieme interventies en het belang van deze interventies.

Concluderend, dit proefschrift heeft vanuit verschillende perspectieven de online Kopstoring cursus geëvalueerd en geeft daarmee nieuw inzicht op het gebied van uitvoer en evaluatie van online interventies. De resultaten zijn van belang voor beleidsmakers en de medisch-wetenschappelijke onderzoek gemeenschap. Ondanks dat de positieve werking van de Kopstoring cursus beschreven is in de proces evaluatie, bleef de klinische statistische bevestiging in de evaluatie studie achter. De resultaten van de studies in dit proefschrift laten het potentieel van online hulpverlening zien en onderstrepen de noodzaak voor toekomstig onderzoek in de kracht en het potentieel van online preventieve interventies. De resultaten van dit proefschrift benadrukken de noodzaak om de discussie aan te gaan over de kloof tussen (bio)medische onderzoek en het wettelijke kader en de organisatorische structuur. Meer dan ooit tevoren dienen we bedacht te zijn op de problemen en obstakels die er zijn rondom de uitvoer van online zorgprogramma's en online evaluatie onderzoek. 



\section{VALORISATION}

"The more extensive a man's knowledge of what has been done, the greater will be his power of knowing what to do."

Benjamin Disraeli (1804-1881) 

This part of the thesis focus on strategies to valorise the results of the conducted research. Valorisation of knowledge is the actual process of making research results suitable and available for the general public and society [1]. The outcome of the evaluation performed has led to new insight and knowledge about online e-research. This chapter will therefore focus on the value of valorisation of these new insights and the possible valorisation strategies.

\section{Relevance for valorisation of knowledge}

Minding the gap

The relevance and importance of valorisation of research results should not be underestimated. Even the result of clinical trials with small effect sizes could be disseminated and valorised. This chapter elaborates on the valorisation strategies for the results of the research performed for this thesis. This thesis explores the potential, and also the effectiveness of Kopstoring, an online preventative course for adolescent offspring of parents who are dealing with mental illness or addiction. Surprisingly, for the research executed within this thesis, it is not the result of the clinical effectiveness study that is most important to valorise, but the knowledge gained about the obstacles encountered when performing this online randomised controlled trial. From the obstacles and problems met during the study, researchers, policy makers, legislators and patients can learn a great deal.

\section{Valorisation strategies}

Bridging the gap

In this part of the valorisation chapter, we propose several strategies to optimize valorisation of the knowledge obtained and in addition the information gathered concerning the problems encountered during the execution of the online randomised clinical trial. A general strategy to valorise results of this thesis could be disseminating results among the scientific community and the community of health care professionals. This process has already started by publishing the results of the studies in International peer reviewed journals. In addition, the results are sent out to the National platform Kopstoring and mental health institutions. Secondly, the results are partly disseminated at National (Amsterdam and Maastricht) and International conferences (Italy, Germany, Canada and the United States of America). In answer to the early dissemination of various studies, Kopstoring attracted a lot of attention and the Karolinska Institute in Sweden has bought the rights to be able to provide Kopstoring in Sweden. At the other side of the world, in Portland, Oregon (USA) experts also see the potential of Kopstoring and are trying to find a platform for negotiations with the developers (Trimbos Instituut and four mental health institutions) to see if there is a potential for the provision of the course in Oregon. 
A second valorisation strategy focuses on valorisation of the knowledge obtained about problems with obtaining both written and online informed consent and the concerns about the age differences for legal decision making for medical treatment and (medical) research. The goal of this strategy is to create optimum awareness about the knowledge gathered because the inclined answer to solve this problem is specifically for National Parliament; the National legislator and policy maker. The first strategy to create awareness and valorise the results of our study on legal issues is one that needs investment. As described in chapter 6 of this thesis, the legal obligation to obtain 'written' informed consent creates serious problems for online research in some cases making it virtually impossible. This claim has been supported by medical and health researchers for some years now, and it has even been noticed and acknowledged by the medical research ethical committee (MReC) and the National Central Committee on Research Involving Humans (CCMO) [2]. Amending an act or legislation is a task that can be performed only by National Parliament [3]. However, since the messages has not been picked up yet it might be time to use more convincing strategies. A first step could be to write a petition which can be served to members of Parliament. Writing a petition is a good strategy to create awareness and build a large community to support this initiative to allow online consent in medical research (in cases where it is deemed absolutely necessary and under strict regulation). If the petition is constructed the correct way it will be understandable, clear and demanding only minimal effort from the supporting party. In accordance to writing a petition a citizens initiative is a second tool which can be used to create awareness and with enough support (online signatures) has a direct impact on the political agenda [4].

In addition to the scientific community and Dutch policy makers and Parliament, the target population would benefit from transparent valorisation and dissemination of the results of this thesis. After all, they are the ones suffering from the situation they are in. The course participants have already indicated how valuable Kopstoring can be. Communicating the results of this evaluation, will hopefully lead to more effective adoption of Kopstoring. As laid out in this thesis, future provision of Kopstoring might be threatened by future re-organisations of the youth mental health care sector and its financial consequences. Valorisation is even more important in that regard, therefore we will disseminate the study results by sending the thesis to health care providers, participants, municipalities and health care insurers. 


\section{REFERENCES}

1. http://www.netherlandsproteomicscentre.nl/npc/valorisation/what-is-valorisation.htmlref.2016 [cited 2016 01-02-2016].

2. CCMO, Besluit CCMO, afgegeven dd. 20 maart 2012 (bes/uit hoorzitting, van januari 2012), in CCMO. 2012: Den Haag.

3. Bellekom, T.I., et al., Compendium Staatsrecht. Vol. 10. 2007: Uitgeverij Kuwer BV. 427.

4. Tweede Kamer van de Staten Generaal), http://www.tweedekamer.nl/kamerleden/commissies/verz/ burgerinitiatieven. 2016 [cited 2016 01-02]. 

APPENDICES 



\section{APPENDIX 1}

\begin{tabular}{|c|c|c|}
\hline $\begin{array}{l}\text { Intervention } \\
\text { FACE-TO-FACE }\end{array}$ & $\begin{array}{l}\text { Population } \\
\text { INTERVENTIONS }\end{array}$ & Aims intervention \\
\hline $\begin{array}{l}\text { Play-and-talk } \\
\text { groups }\end{array}$ & $\begin{array}{l}\text { Children } \\
\text { (12-16 yrs) }\end{array}$ & $\begin{array}{l}\text { Decrease risk factors and } \\
\text { strengthen protective factors by } \\
\text { breaking through social isolation } \\
\text { and the taboo, fostering mutual } \\
\text { recognition, decrease the burden, } \\
\text { and stimulating and searching for } \\
\text { social support }\end{array}$ \\
\hline $\begin{array}{l}\text { Support groups } \\
\text { for adolescents }\end{array}$ & $\begin{array}{l}\text { Children } \\
\text { (16-23 yrs) }\end{array}$ & $\begin{array}{l}\text { Decrease risk factors and } \\
\text { strengthen protective factors } \\
\text { breaking through social isolation } \\
\text { and the taboo, fostering mutual } \\
\text { recognition, decrease the burden, } \\
\text { and stimulating and searching for } \\
\text { social support }\end{array}$ \\
\hline
\end{tabular}

Child Talks Parents\&children Improving the children's coping (0-23 yrs) skills and offering them emotional and social support, improving the parent's competence by increasing their awareness of their children's perspectives, and informing them of the consequences that a parent's mental illness may have for the children

Psychoeducational family intervention
Content intervention

Children receive information about the problems of their parents, get social support from the group, and are trained in strategies to enhance their competence in coping with the problems of their parents ( 1 interview with parent and child, 8 child group meetings, 2 parent group meetings)

Similar as in the play-and-talk groups. Program content is flexible and chosen in consultation with the participants. Common topics: heritability, feelings of guilt and shame, feeling responsible for the parent, leaving home, making plans for the future ( 8 child group meetings)

A routine service of mental health services after the intake of each adult patient who has children living at home. Conversations with trained mental health professional. After the conversations, advice is offered about the additional help and support that is available (1 parent meeting, 2 family meetings) Parents report the history of their situation, are taught about improving the resilience and strength of their children, and concerns are discussed. Information is provided to the children and their concerns are discussed. In a wholefamily meeting, the mental illness, a shared coping strategy, and positive steps to promote healthy child functioning are discussed (6 to 8 family meetings; first with parents, then with children, then together) 


\begin{tabular}{|c|c|c|c|}
\hline $\begin{array}{l}\text { Intervention } \\
\text { FACE-TO-FACE }\end{array}$ & $\begin{array}{l}\text { Population } \\
\text { INTERVENTIONS }\end{array}$ & Aims intervention & Content intervention \\
\hline Piep zei de muis & $\begin{array}{l}\text { Toddlers and } \\
\text { young children } \\
\text { (4-7 yrs) }\end{array}$ & $\begin{array}{l}\text { Teach children playfully how to } \\
\text { deal with their emotions and the } \\
\text { stress and tension in their home } \\
\text { situation. }\end{array}$ & $\begin{array}{l}\text { Young children exposed to } \\
\text { emotional stress and stress. } \\
\text { Focused on children from poor } \\
\text { neighborhoods. Children learn in } \\
\text { the "play club' how to deal with } \\
\text { their emotions and the situation at } \\
\text { home in a playful manner. } 15 \\
\text { meetings and } 4 \text { parent meetings }\end{array}$ \\
\hline $\begin{array}{l}\text { Gezin aan bod / } \\
\text { family'sturn }\end{array}$ & $\begin{array}{l}\text { Families with } \\
\text { parents with } \\
\text { addiction pro- } \\
\text { blems (all ages) }\end{array}$ & $\begin{array}{l}\text { Start a process of communication } \\
\text { between the family members } \\
\text { about the parent's addiction. }\end{array}$ & $\begin{array}{l}\text { Psycho-educational intervention for } \\
\text { the family with separate parts for } \\
\text { child and parents but also part for } \\
\text { both to practice what they learned } \\
\text { as a family. } 14 \text { weekly sessions of } \\
2.5 \text { h. first hour is only for the } \\
\text { children and rest of the time } \\
\text { complete families. }\end{array}$ \\
\hline
\end{tabular}

\begin{tabular}{|c|c|c|c|}
\hline $\begin{array}{l}\text { Intervention } \\
\text { WEBBASED }\end{array}$ & $\begin{array}{l}\text { Population } \\
\text { NTERVENTIONS }\end{array}$ & Aims intervention & Content intervention \\
\hline 'Kopstoring' & $\begin{array}{l}\text { Children } \\
\text { (16-25 yrs) }\end{array}$ & $\begin{array}{l}\text { Improving the mental health of } \\
\text { children, educate children about } \\
\text { their parent's illness, and create } \\
\text { understanding between children } \\
\text { and parents }\end{array}$ & $\begin{array}{l}\text { Themes include describing the } \\
\text { situation at home and roles in the } \\
\text { family; thoughts, feelings, and self- } \\
\text { blame; questions about addiction } \\
\text { and mental problems; coping with } \\
\text { different behaviors; parentification; } \\
\text { using social network; leading your } \\
\text { own life; preparing for your own } \\
\text { future ( } 8 \text { chat sessions) }\end{array}$ \\
\hline ‘KopOpOuders' & Parents & $\begin{array}{l}\text { Support both parents in raising } \\
\text { their children }\end{array}$ & $\begin{array}{l}\text { Topics: the role as a parent, the } \\
\text { impact of parental problems on } \\
\text { children, feelings of guilt and shame, } \\
\text { what is good-enough parenting, } \\
\text { what practical pedagogical support } \\
\text { is available, opportunity to exchange } \\
\text { experiences between participants ( } 8 \\
\text { sessions) }\end{array}$ \\
\hline 'Survivalkid' & $\begin{array}{l}\text { Children } \\
\text { (12-24 yrs) }\end{array}$ & $\begin{array}{l}\text { Provide children opportunities to } \\
\text { find support and communicate } \\
\text { with others any time they feel } \\
\text { the need }\end{array}$ & $\begin{array}{l}\text { Survivalkid provides a secluded } \\
\text { virtual platform with personalized } \\
\text { feedback, psycho-social education, a } \\
\text { message board, monitored chat } \\
\text { groups and opportunities for private } \\
\text { chats with a professional }\end{array}$ \\
\hline
\end{tabular}




\begin{tabular}{|c|c|c|c|}
\hline $\begin{array}{l}\text { Intervention } \\
\text { WEBBASED }\end{array}$ & $\begin{array}{l}\text { Population } \\
\text { NTERVENTIONS }\end{array}$ & Aims intervention & Content intervention \\
\hline ‘Drankjewel’ & $\begin{array}{l}\text { Children with } \\
\text { parents with } \\
\text { alcohol addic- } \\
\text { tion (two cate- } \\
\text { gories: } 12-17 \text { yrs } \\
\text { and } 18 \text { yrs and } \\
\text { older) }\end{array}$ & $\begin{array}{l}\text { Provision of information and links. } \\
\text { Provide easy access to contact } \\
\text { professionals to seek help. }\end{array}$ & $\begin{array}{l}\text { Online platform with facts, } \\
\text { frequently asked questions and links } \\
\text { to active interventions. There are } \\
\text { also options to contact a } \\
\text { professional by e-mail or chat with } \\
\text { an professional }\end{array}$ \\
\hline Familie van... & $\begin{array}{l}\text { Family members } \\
\text { of a mentally ill } \\
\text { or addicted } \\
\text { person (two } \\
\text { categories: } \\
\text { younger than } 24 \\
\text { yr and older than } \\
24 \text { yrs old) }\end{array}$ & $\begin{array}{l}\text { Provision of information and links. } \\
\text { Provide easy access to contact } \\
\text { professionals to seek help. } \\
\end{array}$ & $\begin{array}{l}\text { Online platform with facts, } \\
\text { frequently asked questions and links } \\
\text { to active interventions. There are } \\
\text { also options to contact a } \\
\text { professional by e-mail or chat with } \\
\text { an professional }\end{array}$ \\
\hline
\end{tabular}

*Table based (adapted) on overview from van Loon (2015) 


\section{APPENDIX 2}

\section{Sample Size calculations}

Initial sample size calculation:

We want to be able to detect at least a clinical medium effect size. This corresponds to a standardised mean difference (Cohen's $d$ ) of $d>0.45$ [1]. We will test the hypothesis at a conventional power of $(1$ - beta $)=0.80$ and alpha $=0.05,2$-tailed. For this $n=78$ is required per condition, hence a total of 156 participants. The drop-out rate from the pilot study was 28\% [2]. We therefore need to enrol 156/ (1-0.28) $=214$ participants in the trial.

\section{Adjusted sample size calculation:}

The initial power calculation is based on the hypothesis that groups would be tested with an independent $T$ test. However, analyses will be more efficient when using a covariance analysis in which we use the scores of the Youth Self Report List (YSR) measured at baseline as a covariate in the analyses. Through this correlation of the Youth Self Report List (YSR) between the pre(baseline) and post-measurements we can gain an important power advantage. According to the manual of the Youth Self Report List (YSR) [4] there is a correlation of $r=0.88$ between pre- and post-measurement with an interval of 2 months' time. After 24 months the correlation is $r=0.63$ and 48 months $r=0.45$. It is therefore sound to assume that the correlation of the scores of the Youth Self Report List (YSR) over a period of 6 months is approximately $r=0.70$.

As with the initial power calculation we expect to find a clinical difference of $d=0.45$ or more between both conditions as measured at the post-measurement. This will be tested with a conventional threshold of $\alpha=0.05$ (2-sides) and with a power of $(1-\beta)=$ 0.80. According to STATA [3] (syntax sampsi 0.000 .45 sd1(1) sd2(1) power (0.80) method (ancova) pre(1), post(1) r01(0.70)) we need 40 participants in each condition. Combining the intervention and waiting list control condition adds up to 80 participants. We should compensate for a loss of $28 \%$ drop out with $80 /(1-0.25)=112$ participants.

\section{REFERENCES APPENDIX 2}

1. Lipsey MW, Wilson DB: The efficacy of psychological, educational, and behavioral treatment American Psychologist 1993, 48: 1181-1201.

2. Veen C, Van Der Zanden R: Procesevaluatie Kopstoring. Utrecht: Trimbos Insituut; 2007.

3. STATA; StataCorp. 2013. Stata Statistical Software: Release 13. College Station, TX: StataCorp LP.

4. Verhulst, F, Van der Ende, J. Koot H. Handleiding voor de Youth Self report (YSR), (1997) Rotterdam 


\section{APPENDIX 3}

Table 4: Overview characteristics interviewed expert sample

\begin{tabular}{|c|c|c|c|c|c|}
\hline $\mathrm{R}$ & Country & Specific work field (background) & 2009 & 2011 & Member Check \\
\hline 1. & The Netherlands & Epidemiologist/ HTA* & $x$ & & \\
\hline 2. & The Netherlands & Health Scientist/ HTA* & $X$ & & \\
\hline 3. & The Netherlands & Economist/ HTA* & $\mathrm{X}$ & & \\
\hline 4. & The Netherlands & Epidemiologist/ HTA* & $X$ & & \\
\hline 5. & The Netherlands & Health economist & $x$ & & \\
\hline 6. & The Netherlands & Psychologist & $\mathrm{X}$ & & \\
\hline 7. & The Netherlands & Orthopaedist & $x$ & & \\
\hline 8. & The Netherlands & Anthropologist/ HTA* & $\mathrm{x}$ & & \\
\hline 9. & The Netherlands & Prevention worker $\mathrm{CMHI} *$ & $x$ & & \\
\hline 10. & The Netherlands & Sociologist/ Prevention worker $\mathrm{CMHI}$ * & $\mathrm{X}$ & & \\
\hline 11. & The Netherlands & Psychologist & $x$ & $\mathrm{x}$ & \\
\hline 12. & The Netherlands & Economist & & $\mathrm{x}$ & \\
\hline 13. & The Netherlands & (Clinical) Psychologist & & $\mathrm{x}$ & \\
\hline 14. & The Netherlands & Econometrist & & $x$ & \\
\hline 15. & The Netherlands & Clinical psychologist / Prevention worker $\mathrm{CMHI}$ * & & $\mathrm{X}$ & \\
\hline 16. & United Kingdom & Health economist & $\mathrm{X}$ & & $\mathrm{X}$ \\
\hline 17. & United Kingdom & (Mental) Health economist & $x$ & & \\
\hline 18. & United Kingdom & Health Economist & $x$ & $\mathrm{x}$ & $\mathrm{X}$ \\
\hline 19. & United Kingdom & Health Economist & $\mathrm{X}$ & $\mathrm{X}$ & \\
\hline 20. & United Kingdom & Child psychiatrist/ Clinician & $\mathrm{X}$ & $x$ & \\
\hline 21. & United Kingdom & Health economist & $X$ & $\mathrm{X}$ & \\
\hline 22. & United Kingdom & Health economist & & $X$ & \\
\hline 23. & $\begin{array}{l}\text { United States of } \\
\text { America }\end{array}$ & (Clinical) Psychologist & & $x$ & \\
\hline 24. & $\begin{array}{l}\text { United States of } \\
\text { America }\end{array}$ & Pediatrician/ Clinician & & $x$ & $\mathrm{X}$ \\
\hline 25. & $\begin{array}{l}\text { United States of } \\
\text { America }\end{array}$ & Economist & & $\mathrm{X}$ & \\
\hline 26. & $\begin{array}{l}\text { United States of } \\
\text { America }\end{array}$ & Economist & & $x$ & \\
\hline 27. & $\begin{array}{l}\text { United States of } \\
\text { America }\end{array}$ & (Clinical) Psychologist & & $\mathrm{X}$ & $X$ \\
\hline 28. & $\begin{array}{l}\text { United States of } \\
\text { America }\end{array}$ & Epidemiologist/ Statistician & & $\mathrm{X}$ & \\
\hline 29. & $\begin{array}{l}\text { United States of } \\
\text { America }\end{array}$ & Epidemiologist & & $x$ & \\
\hline
\end{tabular}

* $\mathrm{CMHI}=$ Community mental health institution

* HTA = Health Technology Assessment 


\section{APPENDIX 4}

Table 5: Interview topic list 2009

\begin{tabular}{|c|c|}
\hline TOPICS & DESCRIPTION \\
\hline 1. Unit of analysis (child/ parent/ family) & $\begin{array}{l}\text { Who is the central figure of analysis; of whom we } \\
\text { measure costs and effects? }\end{array}$ \\
\hline 2. Breadth of Service Use & $\begin{array}{l}\text { Should we measure intersectoral service use? } \\
\text { E.g. in the educational and criminal-justice } \\
\text { systems? }\end{array}$ \\
\hline $\begin{array}{l}\text { 3. Services use of relatives, parents, and informal care } \\
\text { givers (indirect costs) }\end{array}$ & $\begin{array}{l}\text { How extensive should we measure service use? } \\
\text { Is it preferable to include service use by parents, } \\
\text { relatives and (informal) care givers? }\end{array}$ \\
\hline 4. School absenteeism/ school 'presenteeism' & $\begin{array}{l}\text { school absenteeism and school 'presenteeism' } \\
\text { (theoretical concept) }\end{array}$ \\
\hline 5. Practicality \& Validity of instruments & $\begin{array}{l}\text { How easy and practical are the available } \\
\text { instruments to use? Validity of instruments }\end{array}$ \\
\hline 6. Hurdles in Youth service use measurement & $\begin{array}{l}\text { All other problems related to service use } \\
\text { research (ethical or practical problems) }\end{array}$ \\
\hline
\end{tabular}




\section{APPENDIX 5}

Table 6: Structured open-end questions expert interviews, 2011

Experts Interviews: Service Use Assessment for Research with Children and Young People Date:

Respondent nr.:

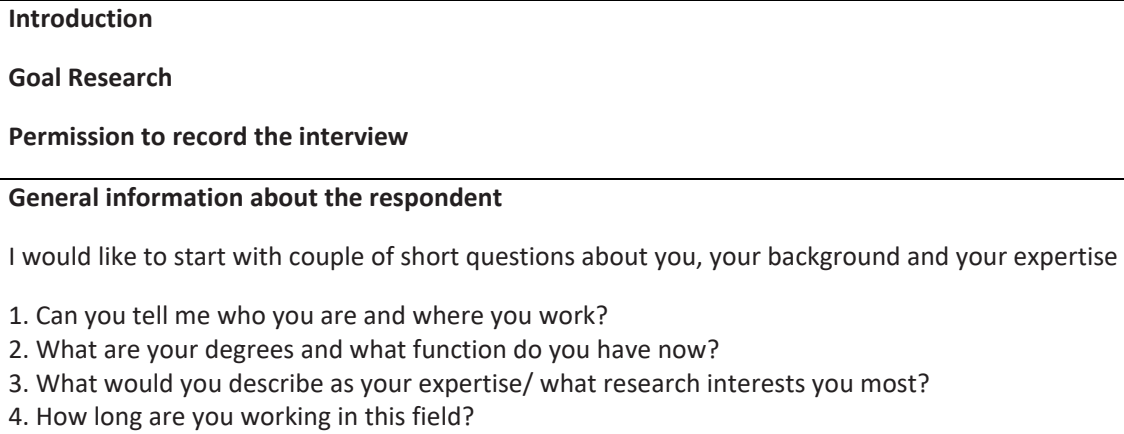

\section{Content}

I would like to ask questions about four themes:
1) Identification of service use data
2) Measurement of service use data
3) Valuation of service use data
4) Hurdles and problems

\section{1) Identification of service use data}

1a. Could you describe what you consider to be identification of service use?

1 b. What are the main issues/ problems with the identification of service use of young people (16-25 years old) in need of mental health care?

1c. What are possible solutions for these issues/ problems, in your view?

Definition of identification:

Identification of service use implies that we are able to discover and identify what (health care) services are used by young people in need of mental health care.

2. Which methods should we use to identify services used by young people in the mental health care sector, in your opinion?

- What methods do you use or have you used in the past?

- Which methods do you prefer and why?

(Eg: diaries, questionnaires, web-based questionnaires, interviews etc)

(Self report or proxy scheme's)

3a. What different perspectives are there to use in service use measurement of young people in need of mental health care? 
- Which one do you use or have you used?
- Which methods do you prefer and why?

$3 b$. Whom should we use as the unit of analysis in service use research and cost research?

Definition perspective:

Societal: Identifying all service used that contribute to burden and costs for society.

Patient: Identifying all services used that contribute to burden and costs for the patient.

Health Care sector/ Insurer: Identifying all services used that contribute to burden and costs for the insurer.

\section{2) Service use measurement}

4a. Can you describe what you consider as measurement of service use?

$4 \mathrm{~b}$. What issues/ problems do you encounter with the measurement of service use data amongst young people in need of mental health care?

4c. What are possible solutions for these problems/ issues, in your opinion?

(E.g respondents, practicality of instruments, vague, don't reach the population, social desirable answers etc)

5. What different methods exist to measure services used by young people in mental health care sector? What methods do you use or have you used?

- Which method do you prefer? Why?

6a. To what extent should we measure service use data of youngsters?

$6 \mathrm{~b}$. With what method? Why?

(Eg. Only health care, also other sectors like juvenile service, criminal and forensic services, school services)

7a. To what extent should we measure the quality of life of informal caregivers? $*$

7b. With what method? Why?

(Rephrase: Is it important to measure quality of life of caregivers in cost-effectiveness analyses or costs of illness studies or to calculate burden of diseases?)

8a. To what extent should we measure school absenteeism?*

8 b. With what method? Why?

(What methods are used now and in what type of schools? What about university, schools after secondary/ high school?)

9a. What do you think about the concept of measuring school presenteeism?*

9b. To what extent should we measure school presenteeism?

9c. With what method? Why?

Definition school presenteeism:

Presenteeism is until now only defined, identified and measured and consequently valued in work settings and not in school settings. Presenteeism in work settings is defined as: being present at work, but working at reduced capacity. It can also be applied to school setting..... being present at school, but working/ performing at reduced capacity. 


\section{3) Valuation of service use data}

10a. Can you describe what you consider as valuation of service used?

$10 \mathrm{~b}$. Which main issues/problems do you encounter with the valuation of service use data amongst youngsters in need of mental health care?

10c. What are possible solutions for these issues/ problems in your opinion?

Definition valuation:

Valuation is to value (e.g in costs) a certain service used. We need valuation in research to express the costs of a certain service use. We can also use this to express the burden of a certain disease.

11. What methods are applicable to valuate services used by young people in mental health care sector?

- What methods do you use, or have you used?

- What is your preferred method? Why?

12a. To what extent should we value service use data? (also other sectors?)

$12 b$. With what method? Why?

13a. How do you want to value service use of the parents in case the child suffers mental illness?

13b. With what method? Why?

\section{4) Hurdles and problems}

14. Do you encounter other issues/ problems in service use research that are not related to identification, measurement or valuation of service use amongst youngsters in need of mental health care?

(Eg. Ethical issues, practical issues, social desirable answers, confidentiality of research, privacy)

15a. In case a child suffers from the illness of the parent (and has increased risks to develop mental illnesses himself) how should we deal with this is service use research?

$15 \mathrm{~b}$. Who is the unit of analysis?

$15 \mathrm{c}$. What is the breadth of service use in this case?

$15 \mathrm{~d}$. What methods do we prefer? Self report methods or proxy methods?

*Authors made a decision not to describe the subject of including quality of life of caregivers, or productivity losses for children and youth as this discussion will be the subject of a separate manuscript. 



\section{DANKWOORD}



Ook voor mij is het zover. Ik ben toegekomen aan het schrijven van het dankwoord. Onwennig is het wel, maar het moment is er. Het behalen van de eindstreep was me niet gelukt zonder de hulp van collega's en vele lieve vrienden om mij heen. Graag wil ik een aantal van deze mensen persoonlijk bedanken.

Allereerst wil ik mijn promotieteam bedanken. Het team was veelzijdig en veerkrachtig, wat nodig bleek te zijn om de uitdagingen van dit project aan te kunnen. Zonder jullie steun, vertrouwen had ik waarschijnlijk de eindstreep niet gehaald. Onno, ik vind je een inspirerend persoon. Je bent me altijd blijven motiveren en inspireren. Ondanks je drukke agenda, mocht ik altijd langskomen en je maakte altijd tijd om te luisteren naar het 'nieuwste probleem' van mijn project. Jij hebt mijn onderzoek en dit proefschrift naar een hoger niveau getild. Ik ben je daar erg dankbaar voor.

Thea wat ben jij een fijn persoon. Je zachte en begripvolle karakter maakte dat ik altijd bij jou durfde aan te kloppen. Ik heb altijd het gevoel gehad dat ik bij jou terecht kon. Je kritische blik, zorgde altijd voor een kwalitatieve verbetering van mijn artikelen. Ik heb heel veel respect voor jou en voor hoe je gelijktijdig verschillende projecten aanpakt en alle ballen in de lucht houdt. Je vervult daarin een voorbeeldfunctie voor mij.

Filip, bedankt voor alle input gedurende mijn project. Je altijd kritische blik en oplossingsgerichtheid was hard nodig dit project. Je bent een bron van ervaring en kennis, die zeer waardevol is. Ik respecteer jou als persoon en waardeer je input enorm. Je hebt het project diepgang gegeven en daar wil ik je dan ook hartelijk voor bedanken.

Voor advies kon ik terecht bij jullie, Silvia en Aggie. Ik wil ook jullie bedanken voor de input van de wetenschappelijke publicaties die de kern van dit proefschrift zijn.

Jennifer Beecham, I am so grateful I had the opportunity to work with you. You are an extremely inspiring and warm person. In addition, I love your sense of humour and the way you motivate students. I sure hope our paths will cross in the future. Thank you Martin Knapp, for the opportunity to work at PSSRU for a while. In this capacity I have learned a great deal about service use research.

Last but not least, Frances Lynch. I really want to thank you for what you have done for me during the last 4 years. You navigated me through the world of qualitative research, service utilization and cost studies. I learned a great deal during my period at Kaiser Permanente. As a person you are so lovely to work with. You have this natural kindness over you that I appreciate very much. I am very happy that our collaboration is yet to be continued and that I have the opportunity to work with you again in the near future. I am looking forward to that!

Leden van de beoordelingscommissie bedankt dat $u$ wilde plaatsnemen en dank voor uw kritische blik op mijn proefschrift 
Op persoonlijk vlak wil ik graag bij het begin beginnen. Arno Verrijth, als ik niet in jouw wiskundeklas terecht was gekomen en eindeloos permutaties en combinaties geoefend had, dan had ik nog steeds een gruwelijke hekel gehad aan elke vorm van wiskunde. Ik ben het echter leuk gaan vinden en dat heb ik wel te danken aan jouw wiskundelessen. Bedankt Bas Verhage en Kim Dirkx, jullie hebben mij in laten zien dat het helemaal niet vanzelfsprekend was een doorstroommaster te kiezen en dat een onderzoeksmaster en onderzoek heel leuk kan zijn. Jullie staan daarmee aan het begin van mijn wetenschappelijke carrière in dat opzicht.

Vladimir jou heb ik gaandeweg leren kennen en we zijn goed bevriend geraakt. Vladimir, altijd even vrolijk, ik hoop dat je nooit stopt met stuiteren want dat maakt jou wie je bent. Saskia, wij zijn van collega's hele goede vriendinnen geworden en dat is super waardevol voor me. Dank voor al je advies en de talloze uurtjes die we binnen en buiten kantooruren gepraat hebben. Ik ben blij dat je tijdens mijn promotie (letterlijk) achter me staat.

Orsida, thank you so much for being such a good friend. I enjoy our long talks about the world, politics, our work and our private lives. Who would have thought that we would become such good friends when they put us in the same office at the London School of Economics. I sure did not, but your friendship is enormously valuable to me. Thanks! Chibuzo, dude, what can I say. I miss you, boss! You just had to move to the other side of the world. I hope we meet again soon in Maastricht. Since you are gone, there is literally no one who has dance moves here in Maastricht. Thanks for your support. Ilse, van alle genoemde personen kennen wij elkaar het langst. We zijn elkaar even uit het oog verloren na de middelbare school, maar ik ben blij dat we elkaar ook weer gevonden hebben. Ondanks dat de wetenschap voor jou een raadsel is, heb je me indirect erg gesteund. Bedankt voor je interesse en hulpvaardigheid en voor onze mooie vriendschap.

Wat hebben we een lief en leed gedeeld, Froukje! Laten we daar vooral mee doorgaan ook nu mijn PhD reis eindelijk ten einde komt. Bedankt voor je luisterend oor, je telefoontjes, en je lieve kaartjes. Karlijn, jou wil ik ook bedanken voor je inzet als coördinator van Kopstoring. Fijn dat we naast werk gerelateerde dingen ook persoonlijke dingen zijn gaan delen. Kevin, dank voor het sparren en voor onze uurtjes bij coffeelovers als we ons even moesten afreageren. Het waren leuke koffie momentjes, die ons beiden uiteindelijk toch weer steeds op het goede pad zette. Daarbovenop bedankt voor de bijzondere vriendschap die we hebben en wat een voorrecht dat ik altijd op jou kan rekenen. We gaan elkaar ook na onze promoties nog veel zien en spreken, hoop ik. Katarina \& Laura, jullie wil ik bedanken voor jullie vriendschap. Beide vriendschappen zijn voortgevloeid uit een samenwerkingsverband. Jullie zijn allebei top meiden en gaan het maken in deze wereld. Bedankt voor de samenwerking binnen CAPHRI, maar minstens zo bedankt voor de vriendschap die eruit voortgekomen is. Jelena, dank je voor onze 'coffee walks' en talloze uurtjes filosoferen. Gaston, jij bent de meest relax- 
te roomy en onderzoeker die ik ken. Het werkt lekker ontnuchterend voor mij dat jij zo lekker chill bent. Volgens mij hebben we best wat 'Wooooosa' momentjes gehad op kantoor. Ik vind het super dat jij mijn paranimf wil zijn. Doreen, jij staat altijd klaar om me uit de brand te helpen. Dank je voor je steun afgelopen jaren. Linda van Loon en Sander van Kuijk jullie verdienen een standbeeld en een heleboel biertjes. Bedankt voor de talloze antwoorden op mijn vragen. Jullie weten me te vinden als jullie de biertjes willen claimen.

Rina, bedankt voor de liefdevolle opvang van Zoe, zonder jouw toewijding en hulp had ik dit proefschrift niet af kunnen maken. Gili \& Ferdie (Aya en Mimi) hetzelfde geldt voor jullie, het zal in de familie zitten. Mede door jullie steun, helpende hand en een dosis liefde, ben ik een enorme pittige periode doorgekomen en op mijn benen blijven staan. Ik waardeer dit enorm en wens jullie al het goeds en liefs toe als familie. Dank je wel!

Jessie (en Ronald), Ik wil ook jou toch graag bedanken voor de steun, vooral in de laatste fase. Ik ervaar je steun als prettig, vertrouwd en fijn! Bedankt, voor de talloze uurtjes die je met Zoe hebt doorgebracht en bedankt voor de geniet en relax momentjes in Breukelen.

Mathijs, grote broer, het zal je een raadsel zijn wat ik doe, maar ik ben erg blij dat je ook op deze dag achter mij staat. Ik beloof dat jij ook na deze dag de slimste van de familie mag blijven ;-)

Niki, jij bent degene die echt weet door te dringen op cruciale momenten en altijd voor me klaarstaat. Dat heb je gedaan al zolang ik je ken. Ik kan onvoorwaardelijk op jouw steun en toewijding rekenen. Vooral in de afrondingsfase heb je me goed, streng en liefdevol bij de les gehouden. Door jouw vertrouwen in mij en ons is dit boekje en project afgerond en is onze eerste stap voor de toekomst gezet. Het is voor ons nu tijd om te genieten van alle mooie dingen die op ons pad gaan komen. Wat hebben wij iets bijzonders en wat hebben we het fijn samen. Ik ben blij met jou!

Zoe, je bent een mooie dochter en een lief meisje. Ik houd van jou.

Lieve papa en mama, het is vreemd om met jullie af te sluiten, maar ik heb het beste voor het laatst bewaard. Ik denk niet dat ik de woorden goed op papier krijg om jullie te bedanken voor de steun die jullie afgelopen jaren geboden hebben. Het zat niet altijd mee, maar ik kan altijd op jullie rekenen. Fijn, dat jullie mij gemotiveerd hebben om door te gaan en mij scherp gehouden hebben om twee studies tegelijk te doen. Het hield daar niet op, jullie zijn me blijven steunen en motiveren om aan dit promotie traject te beginnen en vooral om het af te maken. Het feit dat het nu toch echt af is, daar hebben jullie een heel groot aandeel in gehad. Ik trots op het feit dat ik jullie dochter ben! Met alle liefde; heel veel dank!

\section{Maktub!}



CURRICULUM VITAE 

Marla Woolderink was born on the 8th of April 1984 in Almelo, the Netherlands. She completed her pre-university level (VWO) in 2003 at the Philips van Horne Gemeenschap in Weert, the Netherlands. In 2003, Marla started her bachelor in Public Health at Maastricht University, the Netherlands. During the last phase of the bachelor programme Marla also obtained a minor in Health Law, at the faculty of Law from Maastricht University. In addition, she obtained certificates from all bachelor courses in Dutch Law. In 2007 she obtained her bachelor degree, by completing her thesis about hospital ranking systems in the Netherlands. In 2007, Marla successfully applied to undertake a two-years Health Sciences Research Master at Maastricht University. In 2009, Marla worked at the Personal Social Services Research Unit, at the London School of Economics and Political Sciences, in the United Kingdom. It is there, in London where she finished her Master thesis about service utilization of children and youth in need of mental health care and also finalised her grant research proposal. In 2009 Marla obtained her Master of Philosophy degree and won the grant application procedure. She started working on the project that was laid out in the written grant proposal and initiated her PhD in 2010. In this capacity she has taken on teaching activities, such as PhD and CaRe representatives and took two more international projects for which she visited Ukraine and Georgia (EU). In 2012 Marla spent a period of three months in Portland, Oregon (USA) to work within the health services research group of Kaiser Permanente. During the period of her PhD trajectory, she also presented her work at various international and national conferences. On April $1^{\text {st }}$, Marla started working at Statistics Netherlands as project leader data collection. 


\section{PUBLICATION LIST}

\section{Publications}

Woolderink M, Smit F, van der Zanden R, Beecham J, Knapp M, Paulus A, Evers S (2010) Design of an internet-based health economic evaluation of a preventive groupintervention for children of parents with mental illness or substance use disorders.

BMC Public Health. 2010 Aug 10;10:470. doi: 10.1186/1471-2458-10-470.

Woolderink M, Lynch FL, van Asselt AD, Beecham J, Evers SM, Paulus AT, van Schayck CP (2015) Methodological considerations in service use assessment for children and youth with mental health conditions; issues for economic evaluation.

Administration and Policy in Mental Health and Mental Health Services Research.

2015 May;42(3):296-308. doi: 10.1007/s10488-014-0570-4.

Woolderink M, Bindels J, Evers SM, Paulus AT, van Asselt AD, van Schayck CP (2015)

An online health prevention intervention for youth with addicted or mentally ill parents: experiences and perspectives of participants and providers from a randomized controlled trial.

Journal of Medical Internet Research. 2015 Dec 2;17 (12) doi: 10.2196/jmir.4817.

Woolderink M, Putnik K, van der Boom H, Klabbers G (2015)

THE VOICE OF PhD candidates and PhD supervisors. A qualitative exploratory study amongst $\mathrm{PhD}$ candidates and supervisors to evaluate the relational aspects of $\mathrm{PhD}$ supervision in the Netherlands.

International Journal for Doctoral Studies. 2015 (10).

Woolderink M, van Asselt AD, van Schayck CP, van Wijmen FC (2014)

E-research: problems with anonymity and consent.

Nederlands Tijdschrift voor Geneeskunde. 2013;157(51): A6828.

Boom H, Klabbers G, Putnik K and Woolderink M (2011)

It takes two to tango. A qualitative study amongst $\mathrm{PhD}$ candidates and supervisors to evaluate the process of PhD supervision in the Netherlands.

ISBN: 978-90-5681-416-8. 


\section{Published abstracts}

Woolderink M, Paulus AT, Evers S (2011)

Identifying and measuring service use data for youth in need of mental health care. Psychiatric Praxis. 2011 May;38. doi: 10.1055/s-0031-1277749.

\section{Presentations}

Presentation (keynote speaker): Supervision of PhD candidates and planning of a PhD trajectory. CaRe days, Veldhoven, The Netherlands (2016).

Seminar: Evaluation of an online preventive programme for adolescents with mentally ill or addicted parents. London School of Hygiene and Tropical Medicine (2016).

Oral presentation: Evaluation of an online preventive programme for adolescents with mentally ill or addicted parents. Kaiser Permanente, Center for Health Research, Portland, Oregon, United States of America (2016).

Visiting lecture: Economic evaluation of an online preventative course for offspring at risk. Kings College Hospital London, United Kingdom, Kopstoring project (2015).

Oral presentation: Design of an Internet-Based (Economic) Evaluation of a Preventive Group Intervention for Children of Addicted or Mentally III Parents. International Conference on Child and Youth Mental Health Matters, Vancouver, Canada (2012).

Oral presentation: Identification and Measurement of Service Use Data for Youth in Need of Mental Health Care (Existing Problems and Hurdles). International Conference on Child and Youth Mental Health Matters, Vancouver, Canada (2012).

Oral presentation: Methodological Considerations in Service Use Assessment for Children and Youth with Mental Condition; issues for Economic Evaluation. Kaiser Permanente, Center for Health Research, Portland, Oregon, United States of America (2012).

Oral presentation: Identifying and Measuring Service Use Data for Youth in Need of Mental Health Care. International Conference on Mental Health Policy and Economics, Italy (2011). 
Oral presentation: Identifying and Measuring Service Use Data. European Network for Mental Health Services Evaluation, Ulm, Germany (2011).

Symposium: Health Economic Aspects of Child and Adolescent Mental Health. European Network for Mental Health Services Evaluation, Ulm, Germany (2011).

\section{Societal impact}

Radio interview: L1 (Radio Limburg1) Wat is KOPP/KVO en wat is Kopstoring? Maastricht, The Netherlands (2013).

Workshop: E-health. Week voor de chronisch zieken, (Papendal), Arnhem, the Netherlands (2011).

Krantenartikel: Leren omgaan met een zieke ouder. Observant 17 maart 2011 http://www.interprofessional.ubc.ca/CYMHM/handouts.asp 
